JOURNAL OF THE

AMERICAN MATHEMATICAL SOCIETY

Volume 11, Number 4, October 1998, Pages 799-867

S 0894-0347(98)00275-6

\title{
TWO GEOMETRIC CHARACTER FORMULAS FOR REDUCTIVE LIE GROUPS
}

\author{
WILFRIED SCHMID AND KARI VILONEN
}

\section{INTRODUCTION}

In this paper we prove two formulas for the characters of representations of reductive groups. Both express the character of a representation $\pi$ in terms of the same geometric data attached to $\pi$. When specialized to the case of a compact Lie group, one of them reduces to Kirillov's character formula in the compact case, and the other, to an application of the Atiyah-Bott fixed point formula to the Borel-Weil realization of the representation $\pi$.

To set the stage, let us first recall the Borel-Weil theorem for a connected, compact Lie group $G_{\mathbb{R}}$. For simplicity, we assume that $G_{\mathbb{R}}$ is simply connected. We let $\mathfrak{g}_{\mathbb{R}}$ denote its Lie algebra and $\mathfrak{g}=\mathbb{C} \otimes_{\mathbb{R}} \mathfrak{g}_{\mathbb{R}}$ the complexified Lie algebra. Via the adjoint action, $G_{\mathbb{R}}$ operates on $i \mathfrak{g}_{\mathbb{R}}{ }^{*}$, the space of all $\mathbb{R}$-linear functions $\lambda: \mathfrak{g}_{\mathbb{R}} \rightarrow i \mathbb{R}$. Every $G_{\mathbb{R}^{-}}$orbit $\Omega$ in $i \mathfrak{g}_{\mathbb{R}^{*}}$ - "coadjoint orbit" for short - carries a canonical $G_{\mathbb{R}^{-}}$ invariant symplectic structure. The orbit $\Omega$ is said to be integral if some, or equivalently any, $\lambda \in \Omega$ exponentiates to a character $e^{\lambda}$ of the isotropy subgroup $\left(G_{\mathbb{R}}\right)_{\lambda}$. In that case, the one-dimensional representation spaces of the characters $e^{\lambda}, \lambda \in \Omega$, fit together into a $G_{\mathbb{R}}$-equivariant, real algebraic, Hermitian line bundle $\mathbf{L}_{\Omega} \rightarrow \Omega$. The pair $\left(\Omega, \mathbf{L}_{\Omega} \rightarrow \Omega\right)$ carries a unique "positive polarization": a $G_{\mathbb{R}}$-invariant complex structure on the manifold $\Omega$ and the structure of a $G_{\mathbb{R}}$-equivariant holomorphic line bundle on $\mathbf{L}_{\Omega}$, positive in the sense of algebraic geometry. The hypothesis of simple connectivity ensures that the square root $\sqrt{\mathbf{K}_{\Omega}}$ of the canonical bundle $\mathbf{K}_{\Omega}$ exists as a $G_{\mathbb{R}^{-}}$equivariant holomorphic line bundle. According to the Borel-Weil theorem, the natural action of $G_{\mathbb{R}}$ on the space of holomorphic $\mathbf{L}_{\Omega}$-valued half forms $\mathrm{H}^{0}\left(\Omega, \mathcal{O}\left(\mathbf{K}_{\Omega}^{1 / 2} \otimes \mathbf{L}_{\Omega}\right)\right)$ is irreducible; moreover, the association

$$
\Omega \rightsquigarrow \mathrm{H}^{0}\left(\Omega, \mathcal{O}\left(\mathbf{K}_{\Omega}^{1 / 2} \otimes \mathbf{L}_{\Omega}\right)\right)
$$

sets up a bijection between the integral regular - i.e. maximal dimensional - coadjoint orbits $\Omega \subset i \mathfrak{g}_{\mathbb{R}}$ and the irreducible representations of $G_{\mathbb{R}}$. We should remark that the passage from sections to half forms corresponds to the $\rho$-shift familiar in representation theory.

Still in the setting of a compact group, we write $\Theta_{\Omega}$ for the character of the representation associated to the $\Omega$, and $\theta_{\Omega}$ for the pullback, as half form, of $\Theta_{\Omega}$ to

Received by the editors July 24, 1997.

1991 Mathematics Subject Classification. Primary 22Exx, 55-xx, 14-xx.

The first author was partially supported by the NSF.

The second author was partially supported by the NSA and NSF.

(c)1998 American Mathematical Society 
the Lie algebra $\mathfrak{g}_{\mathbb{R}} ;$ concretely,

$$
\theta_{\Omega}=\left(\operatorname{det} \exp _{*}\right)^{1 / 2} \exp ^{*} \Theta_{\Omega} .
$$

We define the Fourier transform $\hat{\phi}$ of a test function $\phi \in C_{c}^{\infty}\left(\mathfrak{g}_{\mathbb{R}}\right)$ without the usual factor $i$ in the exponent,

$$
\hat{\phi}(\zeta)=\int_{\mathfrak{g}_{\mathbb{R}}} \phi(x) e^{\langle\zeta, x\rangle} d x \quad\left(\zeta \in i \mathfrak{g}_{\mathbb{R}}^{*}\right),
$$

as a function on $i \mathfrak{g}_{\mathbb{R}}^{*}$. Let $\sigma_{\Omega}$ denote the canonical symplectic form and $n$ the complex dimension of $\Omega$. With these conventions, Kirillov's formula can be stated as follows:

$$
\int_{\mathfrak{g}_{\mathbb{R}}} \theta_{\Omega} \phi d x=\frac{1}{(2 \pi)^{n} n !} \int_{\Omega} \hat{\phi} \sigma_{\Omega}^{n}
$$

In effect, the formula expresses the Fourier transform $\hat{\theta}_{\Omega}$ as integration over $\Omega$.

Translation $\ell_{g}: \Omega \rightarrow \Omega$ by a generic $g \in G_{\mathbb{R}}$ has finitely many fixed points. The Atiyah-Bott fixed point formula gives the Lefschetz number

$$
L(g)=\sum_{p}(-1)^{p} \operatorname{tr}\left\{\left(\ell_{g}\right)^{*}: \mathrm{H}^{p}\left(\Omega, \mathcal{O}\left(\mathbf{K}_{\Omega}^{1 / 2} \otimes \mathbf{L}_{\Omega}\right)\right) \rightarrow \mathrm{H}^{p}\left(\Omega, \mathcal{O}\left(\mathbf{K}_{\Omega}^{1 / 2} \otimes \mathbf{L}_{\Omega}\right)\right)\right\}
$$

as a sum over the fixed point set $\Omega^{g}$,

$$
L(g)=\sum_{\zeta \in \Omega^{g}} \frac{\operatorname{tr}\left\{\left(\ell_{g}\right)^{*}:\left(\mathbf{K}_{\Omega}^{1 / 2} \otimes \mathbf{L}_{\Omega}\right)_{\zeta} \rightarrow\left(\mathbf{K}_{\Omega}^{1 / 2} \otimes \mathbf{L}_{\Omega}\right)_{\zeta}\right\}}{\operatorname{det}\left\{\left(\operatorname{Id}-\ell_{g^{*}}\right): T_{\zeta} \Omega \rightarrow T_{\zeta} \Omega\right\}} .
$$

The positivity of the polarization implies the vanishing of the higher cohomology groups $\mathrm{H}^{p}\left(\Omega, \mathcal{O}\left(\mathbf{K}_{\Omega}^{1 / 2} \otimes \mathbf{L}_{\Omega}\right)\right), p>0$. Hence

$$
\Theta_{\Omega}(g)=\sum_{\zeta \in \Omega^{g}} \frac{\operatorname{tr}\left\{\left(\ell_{g^{-1}}\right)^{*}:\left(\mathbf{K}_{\Omega}^{1 / 2} \otimes \mathbf{L}_{\Omega}\right)_{\zeta} \rightarrow\left(\mathbf{K}_{\Omega}^{1 / 2} \otimes \mathbf{L}_{\Omega}\right)_{\zeta}\right\}}{\operatorname{det}\left\{\left(\operatorname{Id}-\left(\ell_{g^{-1}}\right)_{*}\right): T_{\zeta} \Omega \rightarrow T_{\zeta} \Omega\right\}},
$$

for every generic $g \in G_{\mathbb{R}}$. This conclusion translates readily into the Weyl character formula - the Atiyah-Bott fixed point formula provides a geometric interpretation of Weyl's formula.

Our objective in this paper is the generalization of the two formulas (1.4) and (1.5) to the case of a reductive Lie group. When the group is noncompact, no simple, completely explicit character formula is known in general, certainly no formula as simple as the Weyl character formula in the compact case. Our two formulas express the character of any irreducible unitary representation $\pi$ - more generally, of any admissible $\pi$ having an infinitesimal character - explicitly in geometric terms. The formulas, though simple in appearance, do not seem to lead to simple numerical expressions for the values of characters: in effect, the combinatorial complexity of the character values is reflected by the complexity of the geometry.

In the following, we consider a connected, linear, reductive Lie group $G_{\mathbb{R}}$, with Lie algebra $\mathfrak{g}_{\mathbb{R}}$. Such a group has a complexification $G$, whose Lie algebra $\mathfrak{g}$ is naturally isomorphic to the complexification of $\mathfrak{g}_{\mathbb{R}}$. The complex algebraic group $G$ acts algebraically and transitively on the flag variety $X$, i.e., the variety of Borel subalgebras $\mathfrak{b} \subset \mathfrak{g}$. The real group $G_{\mathbb{R}}$ acts real algebraically on $X$, with finitely many orbits. ${ }^{1}$ Representations of $G_{\mathbb{R}}$ can be associated to twisted $G_{\mathbb{R}}$-equivariant sheaves

\footnotetext{
${ }^{1}$ When $G_{\mathbb{R}}$ happens to be compact, it acts transitively, and $X \cong \Omega$ as $G_{\mathbb{R}}$-homogenous complex manifolds, for every regular coadjoint orbit $\Omega \subset i \mathfrak{g}_{\mathbb{R}}{ }^{*}$.
} 
$\mathcal{F}$ on $X[\mathrm{KSd}]$, by an analytic process resembling Beilinson-Bernstein localization [BB1], [BB2]. This analytic construction, which is recalled in detail in $\S 2$, attaches cohomology groups to $\mathcal{F}$, on which $G_{\mathbb{R}}$ acts by translation, continuously with respect to a certain canonical topology. The resulting representations are admissible, and have an infinitesimal character which is determined by the twisting parameter. Every admissible representation with infinitesimal character can be realized in this way, up to infinitesimal equivalence; the realization can be chosen in degree zero, with all other cohomology groups vanishing. Formally, this is analogous to the Borel-Weil realization (1.1): in the compact case, $\mathcal{F}$ reduces to the constant sheaf, and the twisting parameter specifies the line bundle. We write $\Theta(\mathcal{F})$ for the alternating sum of the Harish-Chandra characters of the cohomology groups attached to $\mathcal{F}$. These are conjugation invariant functions on the regular set in $G_{\mathbb{R}}$. The $\Theta(\mathcal{F})$ exhaust the set of characters of admissible representations with infinitesimal character, as follows from what was just said. As in (1.2), the character $\Theta(\mathcal{F})$ has a Lie algebra analogue $\theta(\mathcal{F})$, which determines $\Theta(\mathcal{F})$ at least near the identity.

Our first formula expresses the Fourier transform of $\theta(\mathcal{F})$ as an integral over a certain cycle in the cotangent bundle $T^{*} X$. The idea that this can be done in principle is due to Rossmann, who also worked out a certain special case [R3]. A precise description of the relation between his work and ours, as well as some other references, can be found at the beginning of section $\S 3$ below. Our formula involves the negative of the twisting parameter of $\mathcal{F}$, which we denote by $\lambda$. Rossmann has defined a twisted moment map

$$
\mu_{\lambda}: T^{*} X \longrightarrow \mathfrak{g}^{*},
$$

depending on $\lambda$. When $\lambda$ happens to be regular, $\mu_{\lambda}$ maps $T^{*} X$ real algebraically and isomorphically on $\Omega_{\lambda}=G \cdot \lambda$, the coadjoint orbit through $\lambda$ for the complex group $G$; at the opposite extreme, for $\lambda=0$, the map $\mu_{\lambda}$ reduces to the ordinary moment map, and takes values in the nilpotent cone $\mathcal{N} \subset \mathfrak{g} \cong \mathfrak{g}^{*}$. Let $\phi \in C_{c}^{\infty}\left(\mathfrak{g}_{\mathbb{R}}\right)$ be a test function, and $\hat{\phi}$ its Fourier transform, normalized as in (1.3), but viewed as a holomorphic function on $\mathfrak{g}^{*}$. We can now state our first formula:

$$
\int_{\mathfrak{g}_{\mathbb{R}}} \theta(\mathcal{F}) \phi d x=\frac{1}{(2 \pi i)^{n} n !} \int_{\mathrm{CC}(\mathcal{F})} \mu_{\lambda}^{*} \hat{\phi}\left(-\sigma+\pi^{*} \tau_{\lambda}\right)^{n} .
$$

Here $\sigma$ denotes the canonical (holomorphic) symplectic form on $T^{*} X, \pi^{*} \tau_{\lambda}$ is the pullback from $X$ to $T^{*} X$ of a two-form representing the class determined by $2 \pi i \lambda$ in $\mathrm{H}^{2}(X, \mathbb{C})$, and $n$ the complex dimension of $X$. Most importantly, $\mathrm{CC}(\mathcal{F})$ refers to the characteristic cycle of the sheaf $\mathcal{F}$ as defined by Kashiwara [Ka2]. It is a top-dimensional cycle, of possibly infinite support, carried by $T_{G_{\mathbb{R}}}^{*} X$, the union of conormal bundles of all the $G_{\mathbb{R}}$-orbits on $X$.

For $\mathcal{F}=\mathbb{C}_{X}=$ constant sheaf on $X$, the characteristic cycle $\mathrm{CC}\left(\mathbb{C}_{X}\right)$ coincides with the zero section $X$ in $T^{*} X$; in that case, the formula (1.7) reduces directly to Kirillov's formula (1.4). More generally, for $\lambda$ regular but $\mathcal{F}$ arbitrary, the integration on the right in (1.7) can be performed over the middle-dimensional cycle $\mu_{\lambda}(\mathrm{CC}(\mathcal{F}))$ in the complex coadjoint orbit $\Omega_{\lambda}$,

$$
\int_{\mathfrak{g}_{\mathbb{R}}} \theta(\mathcal{F}) \phi d x=\frac{1}{(2 \pi i)^{n} n !} \int_{\mu_{\lambda}(\operatorname{CC}(\mathcal{F}))} \hat{\phi} \sigma_{\lambda}^{n}
$$

with $\sigma_{\lambda}$ denoting the natural (holomorphic) symplectic form on $\Omega_{\lambda}$. When $\mathcal{F}$ corresponds to a tempered irreducible representation, $\mu_{\lambda}(\mathrm{CC}(\mathcal{F}))$ turns out to be 
homologous to a coadjoint orbit of $G_{\mathbb{R}}$ in $i \mathfrak{g}_{\mathbb{R}}{ }^{*}$, and (1.8) becomes equivalent to Kirillov's "universal character formula" [Ki3] in this special case, which was established by Rossmann [R1]. For nontempered representations of the reductive group $G_{\mathbb{R}}$, the "universal character formula" fails, since there are not enough coadjoint orbits. It was Rossmann's idea to express irreducible characters not necessarily as integrals over coadjoint orbits, but more generally as integrals over cycles in complexified coadjoint orbits. In this sense, the formulas (1.7)-(1.8) can be regarded as remedies for the failure of the "universal character formula" in the reductive case.

Our second formula was conjectured by Kashiwara [Ka4]. It comes in several flavors: global, pointwise, both as formulas on the group and on the Lie algebra. Here, in this introduction, we describe two of the four versions. Let $\tilde{G}$ denote the set of pairs $(g, x)$ with $g \in G$ and $x$ in the fixed point set $X^{g}$. This is naturally a smooth complex algebraic variety. Projection to the first factor defines a map $q: \tilde{G} \rightarrow G$, which is a covering over the regular set in $G$, with covering group equal to the Weyl group $W$. The exponentials $e^{\alpha}$ of the roots $\alpha$ exist as multiple valued holomorphic functions on $G$; when pulled back to $\tilde{G}$, they become globally defined algebraic functions. By the same process, the exponential of the twisting parameter becomes a well defined algebraic function on $\tilde{G}$ when $\lambda$ is integral, and a multiple valued function in general. In particular, this exponential generates a rank one local system $\mathbb{C}_{\lambda} \subset \mathcal{O}_{\tilde{G}}$. Kashiwara, in the case of trivial twisting parameter ${ }^{2}$, associates to the sheaf $\mathcal{F}$ a top-dimensional cycle $c(\mathcal{F})$ in $\tilde{G}_{\mathbb{R}}=q^{-1}\left(G_{\mathbb{R}}\right)$. The crux of the matter is a fixed point formalism, which we extend to the general, twisted case in $\S 5$ below. The result is a top-dimensional cycle $c(\mathcal{F})$ in $\tilde{G}_{\mathbb{R}}$, whose coefficients are not integers, but sections of the local system $\mathbb{C}_{\lambda}$. This is the global version, on the group, of our second formula:

$$
\int_{G_{\mathbb{R}}} \Theta(\mathcal{F}) \phi d g=\int_{c(\mathcal{F})}\left(q^{*} \phi\right) \tilde{\omega} \quad \text { for } \phi \in C_{c}^{\infty}\left(G_{\mathbb{R}}\right) ;
$$

here $\tilde{\omega}$ denotes the holomorphic form on $\tilde{G}$ obtained by complexifying the Haar measure $d g$ of $G_{\mathbb{R}}$ to a holomorphic form $\omega$ of top degree on $G$, and dividing the pull back $q^{*} \omega$ by the function $\prod_{\alpha>0}\left(1-e^{-\alpha}\right)$, which vanishes to first order on the singular locus of $q$.

Letting $\phi$ run through a sequence converging (weakly) to the delta function $\delta_{g}$ at any particular regular $g \in G_{\mathbb{R}}$, we can evaluate both sides of the identity (1.9) on $\phi=\delta_{g}$. The result is a cohomological expression for $\Theta(\mathcal{F})(g)$ in terms of the action of $g$ at its various fixed points - an analogue, in the noncompact case, of (1.5). This local formula for $\Theta(\mathcal{F})$ has a counterpart on the Lie algebra, as does the global formula (1.9). For simplicity, we state only the Lie algebra version of the local formula here, in the introduction - both local versions can be found in $\S 5$.

We consider a regular $\zeta \in \mathfrak{g}_{\mathbb{R}}$, and write $E$ for the connected component of $\zeta$ in the regular set. Each fixed point $x \in X^{\zeta}$ of the infinitesimal action of $\zeta$ - in other words, a zero of $\zeta$ considered as vector field on $X$-determines a pair $(\zeta, x) \in \tilde{\mathfrak{g}}_{\mathbb{R}}$, the Lie algebra analogue of the space $\tilde{G}_{\mathbb{R}}$. This pair, in turn, gives meaning to the value $\alpha_{x}(\zeta)$, for every positive root $\alpha$, and similarly to $\lambda_{x}(\zeta)$. On general grounds,

\footnotetext{
${ }^{2}$ That is, $\lambda=\rho$ in representation theoretic notation. This corresponds to the infinitesimal character of the trivial representation, and makes the twisted sheaves $\mathcal{F}$ into ordinary ones.
} 
there exist constants $d_{E, x}$, such that

$$
\theta(\mathcal{F})(\zeta)=\sum_{x \in X^{\zeta}} \frac{d_{E, x} e^{\lambda_{x}}(\zeta)}{\prod_{\alpha \in \Phi^{+}} \alpha_{x}(\zeta)}
$$

This is Harish-Chandra's local expression for invariant eigendistributions on the Lie algebra. The Lie algebra version of (1.9), evaluated on the delta function at $\zeta$, computes the $d_{E, x}$, and thus makes (1.10a) explicit:

$$
d_{E, x}=\sum_{p}(-1)^{p} \operatorname{dim} \mathrm{H}_{c}^{p}\left(N^{\prime}(\zeta, x) \cap D_{\epsilon}, \mathcal{F}\right), \quad 0<\epsilon \ll 1 .
$$

In this formula, $\mathrm{H}_{c}$ refers to cohomology with compact support, $D_{\epsilon}$ is the $\epsilon$-ball at $x$, and $N^{\prime}(\zeta, x)$ denotes a subspace of $X$ obtained by extending the space of shrinking directions of $\zeta$ at $x$ by means of a suitable unipotent subgroup of $G_{\mathbb{R}}$.

We should point out that the group version of the fixed point formula (1.10) implies, and is implied by, a statement known as Osborne's conjecture. The argument for the equivalence can be found in the note [SV1], which announces our proof of Kashiwara's conjecture. The integral formula (1.7)-(1.8) and its proof were announced in [SV2]; it is a major ingredient of our proof of a conjecture of Barbasch and Vogan [SV3]. The formulas (1.7) and (1.10) provide two radically different expressions for the same quantity $\theta(\mathcal{F})$. One may wonder if it is possible to go directly between them, without the "detour" via representation theory. In the compact case, the equivalence of (1.4) and (1.5) was established by Berline and Vergne, who established their localization formula for this purpose [BV]. The note [S5] speculates on the possibility of deducing (1.10) from (1.7) by a hypothetical localization formula for nonelliptic fixed points.

The proofs of the two formulas follow the same pattern, though the details are very different. We develop them side-by-side; however, they can be read separately. Section 2, which recalls the description of representations in terms of $G_{\mathbb{R}}$-equivariant sheaves, is equally relevant for both formulas. Section 3 develops the statement of the integral formula, and the two subsequent sections, that of the fixed point formula. Both the statement and the proof of the fixed point formula would simplify greatly if we limited ourselves to the case $\lambda=\rho$, as in Kashiwara's statement of his conjecture [Ka4]; his note provides a good introduction to the fixed point formula. The actual proofs occupy $\S \S 7-10$. Except for $\S 8$, which is short, each of the last four sections begins with an introduction common to both formulas, then treats the integral formula, and finally, the fixed point formula.

\section{Geometric DAtA}

Let us begin with the notation and hypotheses that will be in force for the rest of the paper. We fix a connected, complex algebraic, reductive group $G$ which is defined over $\mathbb{R}$. The representations we consider will be representations of a real form $G_{\mathbb{R}}$ of $G$ - in other words, $G_{\mathbb{R}}$ is a subgroup of $G$ lying between the group of real points $G(\mathbb{R})$ and the identity component $G(\mathbb{R})^{0}$. We regard $G_{\mathbb{R}}$ as a reductive Lie group. All of our results remain valid in the larger class of reductive Lie groups considered by Harish-Chandra [HC7, §3]; we shall comment later on certain modifications necessary to cover this larger class of groups: in this section, following the definition of the enhanced flag variety, and in the discussion of standard sheaves in $\S 6$. We pick a maximal compact subgroup $K_{\mathbb{R}}$ of $G_{\mathbb{R}}$ and recall that this is not an 
essential choice: any two maximal compact subgroups are conjugate. The complexification $K$ of $K_{\mathbb{R}}$ lies naturally as a subgroup in $G$. We denote the Lie algebras of the four groups by the corresponding lower case German symbols $\mathfrak{g}, \mathfrak{g}_{\mathbb{R}}, \mathfrak{k}_{\mathbb{R}}$, and $\mathfrak{k}$; the latter three are subalgebras of $\mathfrak{g}$.

By a representation of $G_{\mathbb{R}}$, we shall always mean a continuous representation on a complete, locally convex, Hausdorff topological vector space. Such a representation is called admissible if its restriction to $K_{\mathbb{R}}$ involves any irreducible representation of $K_{\mathbb{R}}$ only finitely often. A representation of $G_{\mathbb{R}}$ is said to have finite length if every increasing chain of closed, invariant subspaces breaks off after finitely many steps. The universal enveloping algebra $\mathcal{U}(\mathfrak{g})$, and hence its center $\mathcal{Z}(\mathfrak{g})$, operates on the dense subspace of all $K_{\mathbb{R}}$-finite vectors of any particular admissible representation $\pi$ of finite length - the so-called Harish-Chandra module of $\pi$. When $\mathcal{Z}(\mathfrak{g})$ acts on the Harish-Chandra module via a character, one says that $\pi$ has an infinitesimal character. Every irreducible, admissible representation $\pi$ does have an infinitesimal character; this follows from the irreducibility of the Harish-Chandra module of $\pi$ [HC2].

A construction of Harish-Chandra attaches a (global) character $\Theta_{\pi}$ to every admissible representation $\pi$ of finite length - a conjugation invariant distribution ${ }^{3}$ on $G_{\mathbb{R}}$. The characters of any set $\Pi$ of irreducible, admissible representations are linearly independent, provided no two representations in $\Pi$ are infinitesimally equivalent. Infinitesimal equivalence, we recall, is the equivalence relation defined by isomorphism of the underlying Harish-Chandra modules. The character is an additive invariant in short exact sequences. It follows that the composition factors of any admissible representation $\pi$ of finite length are determined, up to infinitesimal equivalence, by the character $\Theta_{\pi}[\mathrm{HC} 3]$.

We identify $\mathcal{U}(\mathfrak{g})$ with the algebra of left invariant linear differential operators on $G_{\mathbb{R}}$ via infinitesimal right translation. Under this identification, $\mathcal{Z}(\mathfrak{g})$ corresponds to the algebra of all bi-invariant linear differential operators. As such, $\mathcal{Z}(\mathfrak{g})$ acts on functions and distributions on the group $G_{\mathbb{R}}$. When a representation $\pi$ has an infinitesimal character $\chi$, the algebra $\mathcal{Z}(\mathfrak{g})$ operates on the distribution $\Theta_{\pi}$ via this same character $\chi$. In Harish-Chandra's terminology, $\Theta_{\pi}$ is then an invariant eigendistribution: a conjugation invariant distribution which is an eigenvector for the algebra $\mathcal{Z}(\mathfrak{g})$.

Harish-Chandra's regularity theorem [HC4] asserts that every invariant eigendistribution $\Theta$ is a locally $L^{1}$ function. This locally $L^{1}$ function is real analytic on $G_{\mathbb{R}}^{\prime}$, the set of regular semisimple elements in $G_{\mathbb{R}}$. The complement of $G_{\mathbb{R}}^{\prime}$ has measure zero, so the restriction of $\Theta$ to the open subset $G_{\mathbb{R}}^{\prime} \subset G_{\mathbb{R}}$ completely determines $\Theta$. Consequently, all finite linear combinations of invariant eigendistributions - and in particular all characters of admissible representations of finite length - may be thought of as real analytic functions on $G_{\mathbb{R}}$ with potential singularities along the complement of $G_{\mathbb{R}}^{\prime}$.

The exponential map from $\mathfrak{g}_{\mathbb{R}}$ to $G_{\mathbb{R}}$ is real analytic. Near 0 , it maps $\mathfrak{g}_{\mathbb{R}}{ }^{\prime}$, the set of regular semisimple elements in $\mathfrak{g}_{\mathbb{R}}$, into $G_{\mathbb{R}}$. More precisely,

$$
\begin{gathered}
\exp \left(\mathfrak{g}_{\mathbb{R}}{ }^{\prime} \cap U\right) \subset G_{\mathbb{R}}{ }^{\prime}, \quad \text { where } \\
U=\left\{x \in \mathfrak{g}_{\mathbb{R}} \mid \text { all eigenvalues } \eta \operatorname{of} \operatorname{ad}(x) \text { satisfy }|\operatorname{Im} \eta|<2 \pi\right\} .
\end{gathered}
$$

\footnotetext{
${ }^{3}$ In this paper, "distribution" will be synonymous to "generalized function", i.e., continuous linear functional on the space of smooth, compactly supported measures. Thus, under coordinate changes, distributions transform like functions.
} 
Thus, for any character $\Theta_{\pi}$, we can define

$$
\theta_{\pi}=\sqrt{\operatorname{det}\left(\exp _{*}\right)} \exp ^{*} \Theta_{\pi},
$$

at least as a real analytic function on $\mathfrak{g}_{\mathbb{R}}{ }^{\prime} \cap U$. In fact, the proof of the regularity theorem shows that $\theta_{\pi}$ extends real analytically to all of $\mathfrak{g}_{\mathbb{R}}{ }^{\prime}$. The resulting function is locally $L^{1}$ as a function on $\mathfrak{g}_{\mathbb{R}}$, hence a globally defined distribution - a conjugation invariant distribution, since the exponential map commutes with conjugation. When the representation $\pi$ has an infinitesimal character, $\theta_{\pi}$ is an eigendistribution for $\mathcal{Z}(\mathfrak{g}) \cong S(\mathfrak{g})^{G}$, the algebra of conjugation invariant, constant coefficient differential operators on $\mathfrak{g}_{\mathbb{R}}$ [HC4]. This is the reason for the factor $\sqrt{\operatorname{det}\left(\exp _{*}\right)}$ in (2.1): without it, the preceding statement would not be correct. We shall call $\theta_{\pi}$ the character of $\pi$ on the Lie algebra. It determines $\Theta_{\pi}$ near the identity, at least, since the exponential map is a local diffeomorphism near the origin.

Our character formulas express $\Theta_{\pi}$ and $\theta_{\pi}$ in terms of certain geometric data attached to the representation $\pi$. Let us describe these geometric data. The flag variety $X$ of $\mathfrak{g}$ carries a tautological bundle $\mathcal{B} \rightarrow X$, whose fiber at any $x \in X$ is the Borel subalgebra $\mathfrak{b}_{x} \subset \mathfrak{g}$ which fixes $x$. The various quotients $\mathfrak{b}_{x} /\left[\mathfrak{b}_{x}, \mathfrak{b}_{x}\right]$ are canonically isomorphic, and hence $\mathcal{B} /[\mathcal{B}, \mathcal{B}]$ has a canonical trivialization;

$$
\mathfrak{h}=_{\text {def }} \text { fiber of the trivialized bundle } \mathcal{B} /[\mathcal{B}, \mathcal{B}]
$$

is called the universal Cartan algebra. It comes equipped with a root system $\Phi \subset \mathfrak{h}^{*}$ and an action of the Weyl group $W$. Every concrete Cartan subalgebra $\mathfrak{c} \subset \mathfrak{g}$ has as many fixed points $x \in X$ as the order of $W$; the choice of a particular fixed point $x$ determines concrete isomorphisms

$$
\mathfrak{c} \cong \mathfrak{b}_{x} /\left[\mathfrak{b}_{x}, \mathfrak{b}_{x}\right] \cong \mathfrak{h} .
$$

We specify a notion of positivity in the universal root system $\Phi$ so that the weights of $\mathfrak{g} / \mathfrak{b}_{x}$ constitute the set positive roots $\Phi^{+}$. In analogy to the universal Cartan algebra (2.2), we define the universal Cartan group $H$ for $G$,

$$
H \cong B_{x} /\left[B_{x}, B_{x}\right]
$$

where $B_{x}$ denotes the Borel subgroup of $G$ stabilizing $x$. This group has Lie algebra $\mathfrak{h}$ and contains

$$
Z=\text { center of } G
$$

canonically, i.e., independently of the choice of $x$.

Beilinson-Bernstein [BB3] introduce the enhanced flag variety of $\mathfrak{g}$ as the fiber bundle $\hat{X}_{\mathfrak{g}} \rightarrow X$ whose fiber at $x \in X$ is a collection of generators of the $\alpha$-root spaces in $\mathfrak{g} / \mathfrak{b}_{x}$ for all simple roots $\alpha \in \Phi^{+}$. It is visibly a principal bundle over $X$ with structure group equal to the product of copies of $\mathbb{C}^{*}$ indexed by the simple roots. The universal Cartan group $H$ acts on the simple root spaces, hence on $\hat{X}$, and $Z$ is the kernel of this action. One can therefore identify

$$
H / Z=\text { structure group of } \hat{X}_{\mathfrak{g}} \rightarrow X .
$$

The group $G$ acts equivariantly on $\hat{X}_{\mathfrak{g}} \rightarrow X$, transitively on both $\hat{X}_{\mathfrak{g}}$ and $X$, and the action on $\hat{X}_{\mathfrak{g}}$ commutes with the action of $H$. To see all of this more concretely (but less invariantly), we visualize $X$ as the quotient $G / B_{x}$ and

$$
\hat{X}_{\mathfrak{g}} \cong G /\left(Z N_{x}\right) \cong Z \backslash G / N_{x},
$$


with $N_{x}=$ unipotent radical of $B_{x}$. Here $H$ acts on $\hat{X}_{\mathfrak{g}}$ by

$$
h: g\left(Z N_{x}\right) \mapsto g h^{-1}\left(Z N_{x}\right) .
$$

The surjection $G \rightarrow Z \backslash G$ determines a principal bundle

$$
\hat{X} \rightarrow X \text {, with structure group } H,
$$

which lies over $\hat{X}_{\mathfrak{g}} \rightarrow X$ with fiber $Z$. We shall call $\hat{X}$ the enhanced flag variety of $G$. Via the identification $X \cong G / B_{x}$, we get the description

$$
\hat{X} \cong G / N_{x}, \quad \text { with } H \text {-action } \quad h: g N_{x} \mapsto g h^{-1} N_{x},
$$

analogous to $(2.7 \mathrm{a}, \mathrm{b})$.

In the definition of the twisted equivariant derived category which is about to follow, we shall work with $\hat{X}$, unlike Beilinson-Bernstein [BB3], who use $\hat{X}_{\mathfrak{g}}$ instead. One can show that both choices result in the same equivariant derived category. When $G_{\mathbb{R}}$ is nonlinear and semisimple, as in [BB3], $\hat{X}_{\mathfrak{g}}$ is the better choice, since it is associated to the Lie algebra, rather than a specific complex group with Lie algebra $\mathfrak{g}$. On the other hand, if $G_{\mathbb{R}}$ is linear but with center of positive dimension, $\hat{X}$ is preferable. In the general case, with $G_{\mathbb{R}}$ reductive and not necessarily linear, neither $\hat{X}_{\mathfrak{g}}$ nor $\hat{X}$ is completely satisfactory; instead one may want to work with yet another bundle over $X$, with structure group $\mathfrak{h}$.

In the discussion below, we keep fixed a particular "localization parameter" $\lambda \in \mathfrak{h}^{*}=$ dual space of $\mathfrak{h}$. As is customary, we set

$$
\rho=\frac{1}{2} \sum_{\alpha \in \Phi^{+}} \alpha .
$$

Let us define the " $G_{\mathbb{R}}$-equivariant derived category on $X$ with twist $(\lambda-\rho)$ ", to be denoted by $\mathrm{D}_{G_{\mathbb{R}}}(X)_{\lambda}$. Technically speaking, it is not a derived category of sheaves on $X$, but rather a pre-stack - concretely, a subcategory of the $\left(G_{\mathbb{R}} \times \mathfrak{h}\right)$-equivariant derived category on $\hat{X}$. For $\lambda=\rho, \mathrm{D}_{G_{\mathbb{R}}}(X)_{\rho}=\mathrm{D}_{G_{\mathbb{R}}}(X)$ is the bounded $G_{\mathbb{R}^{-}}$ equivariant derived category in the sense of Bernstein-Lunts [BL]; a short summary of their construction can be found in $[\mathrm{MV}]$. Our shift by $\rho$ serves the purpose of making the labeling compatible with Harish-Chandra's description of the characters of $\mathcal{Z}(\mathfrak{g})$ [HC1].

We let $G_{\mathbb{R}} \times \mathfrak{h}$ act on $\hat{X}$ via the $(G \times H)$-action, the inclusion $G_{\mathbb{R}} \hookrightarrow G$, and the exponential homomorphism exp $: \mathfrak{h} \rightarrow H$. A $\mathbb{C}$-sheaf $\mathcal{F}$ on $\hat{X}$ is said to be $(\lambda-\rho)$ monodromic with respect to the $H$-action on $\hat{X} \rightarrow X$ if it is locally constant on every fiber, with monodromy $e^{\lambda-\rho}$. To clarify what we mean, note that each fiber can be identified with $H$ (canonically up to translation), so the restriction of $\mathcal{F}$ to any fiber pulls back to a locally constant sheaf on $H$. The $(\lambda-\rho)$-monodromicity condition on $\mathcal{F}$ requires that the locally constant sheaves on $H$, corresponding to the various fibers, have the same monodromy as the rank one local system generated by the multiple valued function $e^{\lambda-\rho}$.

The $(\lambda-\rho)$-monodromic sheaves constitute an abelian category $S h_{X, \lambda}$. One can thus form the bounded derived category $\mathrm{D}^{b}\left(S h_{X, \lambda}\right)$ and the full subcategory $\mathrm{D}_{c}^{b}\left(S h_{X, \lambda}\right)$ of complexes whose cohomology is constructible with respect to a subanalytic stratification. We then pass from $\mathrm{D}_{c}^{b}\left(S h_{X, \lambda}\right)$ to the bounded $G_{\mathbb{R}^{-} \text {-equivariant }}$ derived category $\mathrm{D}_{G_{\mathbb{R}}}(X)_{\lambda}$ as described in [BL]. A seemingly different description of the twisted equivariant derived category appears in $[\mathrm{B}],[\mathrm{MUV}]$; one can show that the two definitions agree. 
Often we shall view objects in $\mathrm{D}_{G_{\mathbb{R}}}(X)_{\lambda}$ as complexes of sheaves on $\hat{X}$, disregarding the $G_{\mathbb{R}}$-equivariance. Since $G_{\mathbb{R}} \times H$ acts real algebraically on $\hat{X}$, with finitely many orbits $[\mathrm{Ma}],[\mathrm{W}]$, the orbits define a semi-algebraic (Whitney) stratification of $\hat{X}$. Any $\mathcal{F} \in \mathrm{D}_{G_{\mathbb{R}}}(X)_{\lambda}$, viewed as an element in $\mathrm{D}^{b}\left(S h_{X, \lambda}\right)$, has locally constant cohomology along the orbits, i.e.,

$$
\begin{gathered}
\text { the image of } \mathcal{F} \in \mathrm{D}_{G_{\mathbb{R}}}(X)_{\lambda} \text { in } \mathrm{D}^{b}\left(S h_{X, \lambda}\right) \text { under the forgetful functor } \\
\text { is constructible with respect to the }\left(G_{\mathbb{R}} \times H\right) \text {-orbit stratification. }
\end{gathered}
$$

In particular, the construction of $\mathrm{D}_{G_{\mathbb{R}}}(X)_{\lambda}$ can be carried out in the semi-algebraic context. The twisting disappears when $\lambda=\rho$, hence

$$
\mathrm{D}_{G_{\mathbb{R}}}(X)_{\rho}=\mathrm{D}_{G_{\mathbb{R}}}(X) .
$$

The construction of $\mathrm{D}_{G_{\mathbb{R}}}(X)_{\lambda}$ involves only the monodromy of the multiple valued function $e^{\lambda-\rho}$ on $H$, not the parameter $\lambda$ itself. This implies the periodicity condition

$$
\mathrm{D}_{G_{\mathbb{R}}}(X)_{\lambda}=\mathrm{D}_{G_{\mathbb{R}}}(X)_{\lambda+\mu} \text { if } \mu \in \mathfrak{h}^{*} \text { is } H \text {-integral. }
$$

Bernstein-Lunts [BL] extend the standard operations on derived categories to the equivariant setting, among them Verdier duality operator $\mathbb{D}$. Duality reverses the twisting from $\lambda-\rho$ to $-(\lambda-\rho)=(-\lambda+2 \rho)-\rho$, so

$$
\mathbb{D}: \mathrm{D}_{G_{\mathbb{R}}}(X)_{\lambda} \stackrel{\sim}{\longrightarrow} \mathrm{D}_{G_{\mathbb{R}}}(X)_{-\lambda+2 \rho}=\mathrm{D}_{G_{\mathbb{R}}}(X)_{-\lambda}
$$

because $2 \rho$ is integral.

The geometric description of representations we shall use involves an additional ingredient: $\mathcal{O}_{X}(\lambda)$, the twisted sheaf of holomorphic functions, with twist $(\lambda-\rho)$. Concretely, this is a subsheaf of the sheaf of holomorphic functions on $\hat{X}$, the subsheaf consisting of all germs $f$ whose restriction to the fiber of $\hat{X} \rightarrow X$ is a constant multiple of $e^{\lambda-\rho}$. Here, as before, we identify the fiber with $H$ via the action in (2.9). This definition manifestly depends on the particular value of $\lambda-\rho$, so $\mathcal{O}_{X}(\lambda)$ does not satisfy the periodicity analogous to (2.13). When $\lambda-\rho$ happens to be $H$-integral, the character $e^{\lambda-\rho}$ of $H$ pulls back to the isotropy groups $B_{x}$, and thus determines a $G$-equivariant holomorphic line bundle

$$
\mathbf{L}_{\lambda-\rho} \rightarrow X
$$

Equivalently, $\mathbf{L}_{\lambda-\rho}$ can be described as the line bundle associated to the principal bundle $q: \hat{X} \rightarrow X$ by the character $e^{\lambda-\rho}$ of the structure group $H$. In this case the twisted sheaf $\mathcal{O}_{X}(\lambda)$ becomes an actual sheaf on $X$ with an action of $G$, and as such coincides with the sheaf of holomorphic sections of the equivariant line bundle $\mathbf{L}_{\lambda-\rho}$. We should remark that these statements depend on the presence of $h^{-1}$, rather than $h$, in (2.9).

By construction, the sheaves $\mathcal{O}_{X}(\lambda)$ on $\hat{X}$ are $(\lambda-\rho)$-monodromic. Hence, for $\mathcal{F} \in \mathrm{D}_{G_{\mathbb{R}}}(X)_{\lambda}$, we can define the groups $\operatorname{Ext}^{*}\left(\mathcal{F}, \mathcal{O}_{X}(\lambda)\right)$ by deriving the functor Hom on the category $S h_{X, \lambda}$, which has enough injectives. Equivalently, one can interpret $\mathcal{H} \operatorname{om}\left(\mathcal{F}, \mathcal{O}_{X}(\lambda)\right)$ as a sheaf on $X$, and $\operatorname{define~}_{\operatorname{Ext}}{ }^{(}\left(\mathcal{F}, \mathcal{O}_{X}(\lambda)\right)$ as the cohomology of

$$
R \operatorname{Hom}\left(\mathcal{F}, \mathcal{O}_{X}(\lambda)\right)=R \Gamma\left(X, R \mathcal{H} \text { om }\left(\mathcal{F}, \mathcal{O}_{X}(\lambda)\right)\right) .
$$


The paper $[\mathrm{KSd}]$ defines a natural, functorial Fréchet topology and a continuous, functorial $G_{\mathbb{R}^{-}}$action on $\operatorname{Ext}^{*}\left(\mathcal{F}, \mathcal{O}_{X}(\lambda)\right)$, with the following property:

$$
\operatorname{Ext}^{p}\left(\mathcal{F}, \mathcal{O}_{X}(\lambda)\right) \text { is an admissible } G_{\mathbb{R}} \text {-module of finite length, }
$$

for all $p \in \mathbb{Z}$ and $\mathcal{F} \in \mathrm{D}_{G_{\mathbb{R}}}(X)_{\lambda}$. In particular, this representation has a $G_{\mathbb{R}^{-}}$ character $\Theta\left(\operatorname{Ext}^{p}\left(\mathcal{F}, \mathcal{O}_{X}(\lambda)\right)\right)$ and a $\mathfrak{g}_{\mathbb{R}}$-character $\theta\left(\operatorname{Ext}^{p}\left(\mathcal{F}, \mathcal{O}_{X}(\lambda)\right)\right)$.

The correspondence between $\mathcal{F}$ and the $G_{\mathbb{R}}$-module (2.16) is contravariant. We make it covariant by inserting the Verdier duality operator ${ }^{4}(2.14)$. Taking alternating sums, we define the virtual characters

$$
\begin{aligned}
\Theta(\mathcal{F}) & =\sum_{p}(-1)^{p} \Theta\left(\operatorname{Ext}^{p}\left(\mathbb{D} \mathcal{F}, \mathcal{O}_{X}(\lambda)\right)\right), \\
\theta(\mathcal{F}) & =\sum_{p}(-1)^{p} \theta\left(\operatorname{Ext}^{p}\left(\mathbb{D} \mathcal{F}, \mathcal{O}_{X}(\lambda)\right)\right),
\end{aligned}
$$

which depend covariantly on $\mathcal{F} \in \mathrm{D}_{G_{\mathbb{R}}}(X)_{-\lambda}$. Our geometric character formulas, which will be stated in $\S \S 3$ and 4 , express $\Theta(\mathcal{F})$ and $\theta(\mathcal{F})$ in terms of $\mathcal{F}$. We should remark that the assignments $\mathcal{F} \mapsto \Theta(\mathcal{F})$ and $\mathcal{F} \mapsto \theta(\mathcal{F})$ descend to the K-group of $\mathrm{D}_{G_{\mathbb{R}}}(X)_{-\lambda}$, which is generated by "standard sheaves" - i.e., direct images of equivariant, twisted local systems on orbits. Hence, to define $\Theta(\mathcal{F})$ and $\theta(\mathcal{F})$, it is not absolutely necessary to appeal to the results of [KSd]; instead, one may appeal to the less functorial version of (2.16) proved in [SW].

Let $\mathcal{R}\left(G_{\mathbb{R}}\right)$ denote the category of admissible representations of $G_{\mathbb{R}}$ of finite length, and $\mathcal{R}\left(G_{\mathbb{R}}\right)_{\lambda}$ the full subcategory of representations with infinitesimal character $\chi_{\lambda}$, in Harish-Chandra's notation. It is important to note that

$$
\begin{aligned}
& \text { every } \pi \in \mathcal{R}\left(G_{\mathbb{R}}\right)_{\lambda} \text { is infinitesimally equivalent to } \operatorname{Ext}^{0}\left(\mathbb{D} \mathcal{F}, \mathcal{O}_{X}(\lambda)\right) \\
& \text { for some } \mathcal{F} \in D_{G_{\mathbb{R}}}(X)_{-\lambda} \text {, such that } \operatorname{Ext}^{p}\left(\mathbb{D} \mathcal{F}, \mathcal{O}_{X}(\lambda)\right)=0 \text { if } p \neq 0 .
\end{aligned}
$$

In particular, then, our character formulas apply to all admissible representations of finite length. The assertion (2.18) follows from [KSd, (1.1f)], combined with the Beilinson-Bernstein localization functor [BB1] and the Riemann-Hilbert correspondence [Ka1], [Me]. This process produces a specific $\mathcal{F} \in \mathrm{D}_{G_{\mathbb{R}}}(X)_{-\lambda}$ for any $\pi \in \mathcal{R}\left(G_{\mathbb{R}}\right)_{\lambda}$ : let $V_{\pi}$ be the Harish-Chandra module of $K_{\mathbb{R}}$-finite vectors in the representation space of $\pi$, and $\mathcal{M}$ the (derived) Beilinson-Bernstein localization of $V_{\pi}$ at $\lambda$; we apply the deRham functor to $\mathcal{M}$ (this has the effect of switching the sign of twisting parameter) and then the equivalence of categories $\mathrm{D}_{K_{\mathbb{C}}}(X)_{-\lambda} \cong \mathrm{D}_{G_{\mathbb{R}}}(X)_{-\lambda}$ [MUV]; this, up to a shift in degree, gives the sheaf $\mathcal{F}$.

The K-group of $\mathcal{R}\left(G_{\mathbb{R}}\right)_{\lambda}$ is generated by standard representations, i.e., representations parabolically induced from discrete series representations. A more explicit description of the correspondence (2.18), in the case of standard representations, is crucial both for applications of our formulas and for their proof. We give such an explicit description in the sections below.

\section{The integral Formula}

Let $G_{\mathbb{R}}$ be a unimodular Lie group of type I, $\pi$ an irreducible unitary representation of $G_{\mathbb{R}}$, and $\theta_{\pi}$ the character of $\pi$ on the Lie algebra. Kirillov's "universal character formula" [Ki3] attempts to express the Fourier transform $\hat{\theta}_{\pi}$ as an integral over a coadjoint orbit. Beyond the cases of compact and nilpotent groups, which

\footnotetext{
${ }^{4}$ In defining the Verdier duality (2.14), we think of twisted sheaves as objects on $X$; alternatively, we may think of them as sheaves on $\hat{X}$ and apply Verdier duality there. The two operations coincide except for a shift in degree by the real dimension of the fiber $H$, which is even. Thus, in the definition (2.17), the two interpretations of $\mathbb{D}$ have the same effect.
} 
were established by Kirillov himself [Ki1], [Ki2], the formula has been proved, in full generality, for type I solvable groups [C]. The reductive case is more subtle: Rossmann has shown that the formula applies in the case of tempered representations [R1], but the formula fails for nontempered representations - there are simply not enough coadjoint orbits. Duflo's construction [D2] produces irreducible unitary representations of algebraic groups by induction from representations of reductive groups. When this is combined with Rossmann's formula, it implies the validity of the "universal character formula" for generic irreducible unitary representations of type I algebraic groups.

Rossmann [R3] has proposed a remedy for the failure of the "universal formula" in the reductive case: the Fourier transform $\hat{\theta}$ of any invariant eigendistribution $\theta$ on $\mathfrak{g}_{\mathbb{R}}$, with regular infinitesimal character, can be expressed as an integral over an appropriate cycle in a coadjoint orbit for the complexification $G$. Our result is an explicit Rossmann type formula for the character of any admissible representation of finite length, unitary or not, with regular or singular infinitesimal character.

Let us recall the definition of Rossmann's twisted moment map [R3]. For details we refer the reader to [SV4, section 8], which was written with the present application in mind. We fix a compact real form $U_{\mathbb{R}} \subset G$ which contains $K_{\mathbb{R}}$. The twisted moment map corresponding to any particular $\lambda \in \mathfrak{h}^{*}$,

$$
\mu_{\lambda}: T^{*} X \longrightarrow \mathfrak{g}^{*},
$$

is defined as follows. Each $x \in X$ is fixed by a unique maximal torus $T_{\mathbb{R}} \subset U_{\mathbb{R}}$; its complexified Lie algebra $\mathfrak{t}$ becomes canonically isomorphic to the universal Cartan algebra $\mathfrak{h}$ via $\mathfrak{t} \cong \mathfrak{b}_{x} /\left[\mathfrak{b}_{x}, \mathfrak{b}_{x}\right] \cong \mathfrak{h}$. This identification makes $\lambda$ correspond to a $\lambda_{x} \in \mathfrak{t}^{*}$, which we extend to a linear functional on $\mathfrak{g}$ by means of the canonical splitting $\mathfrak{g}=\mathfrak{t} \oplus[\mathfrak{t}, \mathfrak{g}]$. The map $x \mapsto \lambda_{x} \in \mathfrak{g}^{*}$ is real algebraic and $U_{\mathbb{R}^{-} \text {-equivariant, }}$ and the ordinary moment map $\mu: T^{*} X \rightarrow \mathfrak{g}^{*}$ is even complex algebraic and $G$ equivariant, so

$$
\mu_{\lambda}(x, \xi)=\lambda_{x}+\mu(x, \xi) \quad\left(\xi \in T_{x}^{*} X\right)
$$

is a $U_{\mathbb{R}}$-equivariant, real algebraic map. The $G$-orbit through $\lambda_{x}$ depends only on $\lambda$, not on $x$, so we denote it by $\Omega_{\lambda}$. The correspondence $\lambda \mapsto \Omega_{\lambda}$, we note, induces a bijection between $W \backslash \mathfrak{h}^{*}$ and the set of semisimple coadjoint orbits for $G$. When $\lambda$ is regular, $\mu_{\lambda}$ takes values in $\Omega_{\lambda}$, and

$$
\mu_{\lambda}: T^{*} X \stackrel{\sim}{\longrightarrow} \Omega_{\lambda}
$$

is a real algebraic isomorphism. At the opposite extreme, for $\lambda=0$, the twisted moment map reduces to the ordinary moment map, of course.

Both the cotangent bundle $T^{*} X$ and the coadjoint orbit $\omega_{\lambda}$ come equipped with canonical, complex algebraic, $G$-invariant symplectic forms, $\sigma$ and $\sigma_{\lambda}$, respectively.

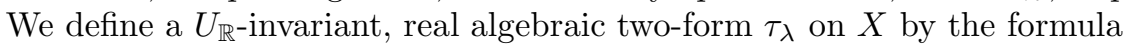

$$
\tau_{\lambda}\left(u_{x}, v_{x}\right)=\lambda_{x}([u, v])
$$

here $u_{x}, v_{x} \in T_{x} X$ are the tangent vectors at $x$ induced by $u, v \in \mathfrak{u}_{\mathbb{R}}$ via differentiation of the $U_{\mathbb{R}}$-action. When $\lambda \in \mathfrak{h}^{*}$ happens to be integral, $-\tau_{\lambda}$ is the curvature form of the (essentially unique) $U_{\mathbb{R}}$-invariant metric on the $G$-invariant algebraic line bundle $\mathbf{L}_{\lambda} \rightarrow X$ parametrized by $\lambda \in \mathfrak{h}^{*}$ [GS]. Moreover, in this situation, $(2 \pi i)^{-1} \tau_{\lambda}$ represents the Chern class of $\mathbf{L}_{\lambda}$. Whenever $\lambda$ is regular, the three differential forms $\sigma, \sigma_{\lambda}, \tau_{\lambda}$ are related. Let $\pi: T^{*} X \rightarrow X$ denote the natural projection; then: 
3.3 Proposition. For $\lambda$ regular, $\mu_{\lambda}^{*} \sigma_{\lambda}=-\sigma+\pi^{*} \tau_{\lambda}$.

Proof. The inverse image $\mu^{-1}(\Omega)$ of the regular nilpotent coadjoint orbit $\Omega$ is dense in $T^{*} X$, so it suffices to verify the identity on $\mu^{-1}(\Omega)$. Let $\sigma_{\Omega}$ be the canonical $G$-invariant, complex algebraic symplectic form on $\Omega$. Rossmann [R3, 7.2, p.172] proves the identity

$$
\mu_{\lambda}^{*} \sigma_{\lambda}=\mu^{*} \sigma_{\Omega}+\pi^{*} \tau_{\lambda} \quad \text { on } \mu^{-1}(\Omega) ;
$$

on the other hand, by [SV4, 8.19],

$$
\mu^{*} \sigma_{\Omega}=-\sigma \quad \text { on } \mu^{-1}(\Omega) .
$$

We should remark that both identities hold for any nilpotent coadjoint orbit $\Omega$, at the smooth points of $\mu^{-1}(\Omega)$. The proofs of this more general version of the two identities in [R3], [SV4] simplify considerably in our more special situation. The two identities together imply the proposition.

The $G_{\mathbb{R}}$-action on the flag variety $X$ is real algebraic and has finitely many orbits. The resulting orbit stratification of $X$ is semi-algebraic and satisfies the Whitney conditions. Hence

$$
T_{G_{\mathbb{R}}}^{*} X={ }_{\text {def }} \text { union of the conormal bundles of the } G_{\mathbb{R}} \text {-orbits }
$$

is a closed, semi-algebraic subset of $T^{*} X$. Like any finite union of conormal bundles, $T_{G_{\mathbb{R}}}^{*} X$ is Lagrangian (at its smooth point) with respect to the canonical symplectic structure on $T^{*} X$, viewed as the $C^{\infty}$ (not holomorphic!) cotangent bundle of $X$. Equivalently this is the symplectic structure defined by the real two-form ${ }^{5} 2 \operatorname{Re} \sigma$. In particular, if $n$ denotes the complex dimension of $X, T_{G_{\mathbb{R}}}^{*} X$ has dimension $2 n$. We write $\mathrm{H}_{*}^{\text {inf }}\left(T_{G_{\mathbb{R}}}^{*} X, \mathbb{Z}\right)$ for the homology of $T_{G_{\mathbb{R}}}^{*} X$ with infinite support - i.e., for the Borel-Moore homology. Then $\mathrm{H}_{2 n}^{i n f}\left(T_{G_{\mathbb{R}}}^{*} X, \mathbb{Z}\right)$ is the group of top-dimensional, possibly infinite cycles on $T_{G_{\mathbb{R}}}^{*} X$.

Kashiwara's characteristic cycle construction [Ka2], [KSa], [SV4] defines a $\mathbb{Z}$ linear map

$$
\mathrm{CC}: K\left(\mathrm{D}_{G_{\mathbb{R}}}(X)_{\lambda}\right) \longrightarrow \mathrm{H}_{2 n}^{i n f}\left(T_{G_{\mathbb{R}}}^{*} X, \mathbb{Z}\right),
$$

from the $K$-group of the twisted equivariant derived category $\mathrm{D}_{G_{\mathbb{R}}}(X)_{\lambda}$. Strictly speaking, the cited references deal only with the untwisted case. However, the characteristic cycle construction is local with respect to the base manifold $X$, and locally twisted sheaves "can be untwisted" - they can be identified with sheaves - so CC makes sense also for twisted sheaves. Alternatively but equivalently, we may view any object $\mathcal{F} \in \mathrm{D}_{G_{\mathbb{R}}}(X)_{\lambda}$ as a monodromic sheaf on $\hat{X}$ and take its characteristic cycle $\mathrm{CC}_{\hat{X}}(\mathcal{F})$ in $T^{*} \hat{X}$; because of the monodromicity condition, $\mathrm{CC}_{\hat{X}}(\mathcal{F})$ is the pullback of a Lagrangian cycle in $T^{*} X$, namely $\mathrm{CC}(\mathcal{F})$. The map $\mathrm{CC}$ becomes intrinsic only when one fixes an orientation of the base manifold $X$. We use the complex structure on $X$ to do so.

Like Rossmann [R3], we define the Fourier transform $\hat{\phi}$ of a test function $\phi$ in $C_{c}^{\infty}\left(\mathfrak{g}_{\mathbb{R}}\right)$ without choosing a square root of -1 , as a holomorphic function on $\mathfrak{g}^{*}$,

$$
\hat{\phi}(\zeta)=\int_{\mathfrak{g}_{\mathbb{R}}} e^{\zeta(x)} \phi(x) d x \quad\left(\zeta \in \mathfrak{g}^{*}\right) .
$$

\footnotetext{
${ }^{5}$ There are at least two different but equally natural identifications between the holomorphic and the $C^{\infty}$ cotangent bundles; we use the convention of [KSa, Chapter XI].
} 
Then $\hat{\phi}$ decays rapidly in the imaginary directions. The particular normalization of the Euclidean measure $d x$ on $\mathfrak{g}_{\mathbb{R}}$ will not matter in the end. One may choose to think of the Fourier transform more invariantly as attached to a smooth compactly supported measure; we leave it to the reader to reinterpret our character formula in these terms.

3.7 Proposition. For all $\phi \in C_{c}^{\infty}\left(\mathfrak{g}_{\mathbb{R}}\right), C \in \mathrm{H}_{2 n}^{i n f}\left(T_{G_{\mathbb{R}}}^{*} X, \mathbb{Z}\right)$, and $\lambda \in \mathfrak{h}^{*}$, the integral

$$
\int_{C} \mu_{\lambda}^{*} \hat{\phi}\left(-\sigma+\pi^{*} \tau_{\lambda}\right)^{n}
$$

converges absolutely. The value of the integral depends holomorphically on $\lambda$.

Rossmann [R3], in the case of a regular $\lambda \in \mathfrak{h}^{*}$, states and proves the convergence and the holomorphic behavior of the corresponding (via 3.3) integral on $\mu_{\lambda}(C) \subset \Omega_{\lambda}$. Whether or not $\lambda$ is regular, this comes down to the rapid decay of the holomorphic function $\hat{\phi}$ in the imaginary directions, as will become clear at the end of this section where we establish a more general convergence criterion.

Recall the definition $(2.17)$ of the virtual character $\theta(\mathcal{F})$. It is a generalized function, and as such can be integrated against any smooth, compactly supported test function $\phi$.

3.8 Theorem. Let $\lambda \in \mathfrak{h}^{*}$ and $\mathcal{F} \in \mathrm{D}_{G_{\mathbb{R}}}(X)_{-\lambda}$ be given. Then

$$
\int_{\mathfrak{g}_{\mathbb{R}}} \theta(\mathcal{F}) \phi d x=\frac{1}{(2 \pi i)^{n} n !} \int_{\mathrm{CC}(\mathcal{F})} \mu_{\lambda}^{*} \hat{\phi}\left(-\sigma+\pi^{*} \tau_{\lambda}\right)^{n} \quad\left(\phi \in C_{c}^{\infty}\left(\mathfrak{g}_{\mathbb{R}}\right)\right) .
$$

Appearances notwithstanding, the formula has canonical meaning, independent of the particular choice of $i=\sqrt{-1}$. We mentioned already that the orientation of $\mathrm{CC}(\mathcal{F})$ depends on the choice of an orientation of $X$. When we orient the complex manifold $X$, we use the complex structure operator $J$, which reflects the choice of $i=\sqrt{-1}$. This same choice affects also the sign on the right hand side of our formula, as it must.

In effect, the theorem provides several integral formulas for any virtual character, corresponding to the various choices of the localization parameter $\lambda$ in any particular $W$-orbit. When $\lambda$ is regular, we can use the isomorphism (3.1a) and Proposition 3.3 to rewrite our formula on the complex, regular, elliptic coadjoint orbit $\Omega_{\lambda}$,

$$
\int_{\mathfrak{g}_{\mathbb{R}}} \theta(\mathcal{F}) \phi d x=\frac{1}{(2 \pi i)^{n} n !} \int_{\mu_{\lambda}(\mathrm{CC}(\mathcal{F}))} \hat{\phi} \sigma_{\lambda}^{n} .
$$

Rossmann [R3] shows that any invariant eigendistribution $\theta$ with regular infinitesimal character can be written as an integral of this type, over an unspecified cycle $C \in \mathrm{H}_{2 n}^{i n f}\left(T_{G_{\mathbb{R}}}^{*} X, \mathbb{C}\right)$. He identifies the cycle explicitly under the following circumstances: $G_{\mathbb{R}}$ is a complex Lie group, and $\lambda$ is integral, regular, and dominant (with Rossmann's conventions, anti-dominant).

The integrand on the right in (3.9) is a holomorphic $2 n$-form, hence is closed, which gets integrated over an (infinite) $2 n$-cycle in the $4 n$-manifold $\Omega_{\lambda}$. As Rossmann [R3] points out, the integral remains unchanged when one replaces the cycle of integration $C_{0}$ by another cycle $C_{1}$, homologous to $C_{0}$ under an appropriately restricted notion of homology which takes into account the growth of cycles and chains at infinity. This observation plays a crucial role in our proof of Theorem 
3.8. When $\theta(\mathcal{F})$ is a tempered irreducible character, with $\lambda$ regular and appropriately chosen within its $W$-orbit, the cycle $\operatorname{CC}(\mathcal{F})$ turns out to be homologous in the restricted sense to the $\mu_{\lambda}^{-1}$-image of a $G_{\mathbb{R}^{-}}$orbit in $i \mathfrak{g}_{\mathbb{R}}^{*}$. In this particular case, then, the integral formula (3.9) is equivalent to the "universal formula" as proved by Rossmann [R1] - our justification for viewing 3.8 as an appropriate analogue of Kirillov's formula.

We need to be more precise about the restricted notion of homology. Initially, we consider a real algebraic manifold $M$ of dimension $m$ and a $C^{\infty}$ differential form $\omega$ on $M$, of degree $d$; $\omega$ may be real or complex valued. Recall the notion of a semi-algebraic, locally finite $d$-chain $C$ [SV4]. We want to define the $\omega$-norm $\|C\|=\|C\|_{\omega}$ in case $|C|=$ support of $C$ is compact - which makes $C$ finite. Thus we can express $C$ as a finite integral linear combination

$$
C=\sum_{j=1}^{N} n_{j} S_{j}
$$

of pairwise disjoint, connected, smooth, oriented, relatively compact, $d$-dimensional semi-algebraic sets $S_{j}$. These hypotheses ensure that

$$
\|C\|=\sum_{j=1}^{N}\left|n_{j}\right| \int_{S_{j}}|\omega|
$$

is well defined and independent of the particular choice of the expression (3.10). In this defining formula, the absolute value $|\omega|$ of the form $\omega$ is viewed as a measure on each of the $S_{j}$.

Let us apply the preceding discussion to the case of $M=T^{*} X$, with $\omega=$ $\left(-\sigma+\pi^{*} \tau_{\lambda}\right)^{n}$ and $d=2 n$. We equip $X$ with a $U_{\mathbb{R}}$-invariant hermitian metric. Any such metric is real algebraic, since $U_{\mathbb{R}}$ acts transitively on $X$. We define

$$
D(r)=\left\{(x, \xi) \in T^{*} X \mid\|\xi\| \leq r\right\},
$$

the disc bundle of radius $r$ in $T^{*} X$; here $\|\xi\|$, the norm of a cotangent vector $\xi$, is measured with respect to the Hermitian metric. The particular choice of metric will not matter, since any two are mutually bounded. For convenience, we choose it so that

$$
\mu: T_{x}^{*} X \longrightarrow \mathfrak{g}^{*} \text { is an isometry for each } x \in X
$$

with respect to some $U_{\mathbb{R}}$-invariant inner product on $\mathfrak{g}^{*}$. We consider a locally finite, semi-algebraic $2 n$-chain $C$. Since $D(r)$ is compact semi-algebraic, the intersection $C \cap D(r)$ can be viewed as a $2 n$-chain ${ }^{6}$ with compact support. Then $C$ has polynomial growth with respect to $\omega=\left(-\sigma+\pi^{*} \tau_{\lambda}\right)^{n}$, in the sense that

$$
\|C \cap D(r)\| \text { grows at most polynomially with } r \text {. }
$$

To see this, we compactify $T^{*} X$ by regarding it as a real algebraic $\mathbb{R}$-vector bundle with distinguished Riemannian metric, hence with structure group $O(2 n, \mathbb{R})$. The linear action of $O(2 n, \mathbb{R})$ on $\mathbb{R}^{2 n}$ extends to an algebraic action on the one point compactification $S^{2 n}$. Thus, replacing the typical fiber $\mathbb{R}^{2 n}$ by $S^{2 n}$, we obtain a real algebraic compactification $\overline{T^{*} X}$ of $T^{*} X$. The function $r^{-1}$ extends real algebraically to the complement of the 0 -section in $\overline{T^{*} X}$, and the extended function vanishes to

\footnotetext{
${ }^{6}$ Stratify $|C|=$ support of $C$ compatibly with its intersection with $D(r)$; then each $2 n$-stratum in $|C| \cap D(r)$ inherits a multiplicity from $C$.
} 
first order on the locus at infinity. Since $\omega$ is real algebraic on $T^{*} X, r^{-k} \omega$ extends real algebraically across the locus at infinity for $k$ sufficiently large - in fact, for $k \geq n$, though the precise value will not matter to us. The chain $C$, being semialgebraic, extends to a finite chain in $\overline{T^{*} X}$, and this now implies (3.14).

Now let $\phi \in C_{c}^{\infty}\left(\mathfrak{g}_{\mathbb{R}}\right)$ be a test function. The Fourier transform $\hat{\phi}$ as defined in (3.6) is holomorphic on $\mathfrak{g}^{*}$ and decays rapidly in imaginary directions; the rate of decay can be uniformly bounded as long as the real part of the argument is restricted to a compact set (Paley-Wiener theorem). It is clear from the definition of the twisted moment map that $\mu_{\lambda}$ differs from the ordinary moment map $\mu$ by a term whose norm can be bounded by a constant multiple of the norm of $\lambda$. Hence, for every $N \in \mathbb{N}$ and $R>0$, there exists a constant $A=A(\phi, N, R)$, such that

$$
\left|\mu_{\lambda}^{*} \hat{\phi}(x, \xi)\right| \leq \frac{A}{1+\|\xi\|^{N}} \quad \text { if } \quad\|\operatorname{Re} \mu(x, \xi)\|+\|\lambda\| \leq R .
$$

The values of the differential form $\mu_{\lambda}^{*} \hat{\phi}\left(-\sigma+\pi^{*} \tau_{\lambda}\right)^{n}$ at the various points in $T^{*} X$ depend holomorphically on $\lambda$, and the polynomial growth condition (3.14), for any particular semi-algebraic $2 n$-chain $C$, holds locally uniformly in $\lambda$. We conclude:

3.16 Lemma. If the real part of $\mu(x, \xi)$ is bounded on the support $|C|$, the integral

$$
\int_{C} \mu_{\lambda}^{*} \hat{\phi}\left(-\sigma+\pi^{*} \tau_{\lambda}\right)^{n}
$$

converges absolutely. The value of this integral depends holomorphically on the parameter $\lambda$.

The moment map $\mu$ takes purely imaginary values on any chain supported on $T_{G_{\mathbb{R}}}^{*} X-$ in fact,

$$
T_{G_{\mathbb{R}}}^{*} X=\mu^{-1}\left(i \mathfrak{g}_{\mathbb{R}}^{*}\right),
$$

as is easy to see; e.g., in [R3]. Thus 3.16 implies Proposition 3.7.

We now consider two semi-algebraic $2 n$-cycles $C_{1}, C_{2}$ in $T^{*} X$, both of which satisfy the boundedness hypothesis of Lemma 3.16. We suppose that $C_{1}$ and $C_{2}$ are homologous, in the sense that

$$
C_{1}-C_{2}=\partial \tilde{C}
$$

for some semi-algebraic $(2 n+1)$-chain $\tilde{C}$. We require that

$$
\text { the image of the support }|\tilde{C}|
$$

under the map $(x, \xi) \mapsto \operatorname{Re} \mu(x, \xi)$ is bounded .

3.19 Lemma. Under the hypotheses (3.18),

$$
\int_{C_{1}} \mu_{\lambda}^{*} \hat{\phi}\left(-\sigma+\pi^{*} \tau_{\lambda}\right)^{n}=\int_{C_{2}} \mu_{\lambda}^{*} \hat{\phi}\left(-\sigma+\pi^{*} \tau_{\lambda}\right)^{n} .
$$

Proof. Let us argue first of all that the $2 n$-form $\mu_{\lambda}^{*} \hat{\phi}\left(-\sigma+\pi^{*} \tau_{\lambda}\right)^{n}$ on $T^{*} X$ is closed. It suffices to show this when $\lambda$ is regular, since the form depends real analytically on $\lambda$. In the regular case,

$$
\mu_{\lambda}^{*} \hat{\phi}\left(-\sigma+\pi^{*} \tau_{\lambda}\right)^{n}=\mu_{\lambda}^{*}\left(\hat{\phi} \sigma_{\lambda}^{n}\right)
$$


is the pullback to $T^{*} X$ of a holomorphic form of top degree, $\hat{\phi} \sigma_{\lambda}^{n}$, on the complex manifold $\Omega_{\lambda}$. As such, it is closed. We restrict the homology relation (3.18a) to the disc bundle $D(r)$ :

$$
C_{1} \cap D(r)-C_{2} \cap D(r)=\partial(\tilde{C} \cap D(r))-C_{3}(r),
$$

with $C_{3}(r)$ supported on the boundary $\partial D(r)$. Thus Stokes' theorem implies

$$
\begin{gathered}
\int_{C_{1} \cap D(r)} \mu_{\lambda}^{*} \hat{\phi}\left(-\sigma+\pi^{*} \tau_{\lambda}\right)^{n}-\int_{C_{2} \cap D(r)} \mu_{\lambda}^{*} \hat{\phi}\left(-\sigma+\pi^{*} \tau_{\lambda}\right)^{n} \\
=-\int_{C_{3}(r)} \mu_{\lambda}^{*} \hat{\phi}\left(-\sigma+\pi^{*} \tau_{\lambda}\right)^{n}
\end{gathered}
$$

so the lemma will follow if the term on the right can be made arbitrarily small as $r$ tends to infinity. Arguing as in the proof of Lemma 3.16, we use (3.15) and (3.18b) to remove $\mu_{\lambda}^{*} \hat{\phi}$ from the picture: we only need to show that the $\omega$-norm

$$
\|\tilde{C} \cap \partial D(r)\| \quad \text { grows at most polynomially with } r \text { as } r \rightarrow \infty .
$$

In interpreting this statement, we need to eliminate the - finitely many - values of $r$ for which the intersection $\tilde{C} \cap \partial D(r)$ fails to be transverse. Note that the complement of the 0 -section in $\overline{T^{*} X}$ is naturally isomorphic to $T^{*} X$; the isomorphism transforms the semi-algebraic chain $\tilde{C}$ into another semi-algebraic chain, the function $r$ to $r^{-1}$, and the real algebraic form $\omega$ to a form with a pole of finite order along the (new) zero-section. We remove the pole by multiplying the form with an appropriate power of the (new) function $r$. Thus, changing notation, we only need to show that

$$
\|\tilde{C} \cap \partial D(r)\| \quad \text { remains bounded as } r \rightarrow 0
$$

when we measure the size of the chain $\tilde{C} \cap \partial D(r)$ with respect to a form which is algebraic around the 0 -section.

To establish (3.23), one can argue as follows. The problem is local around the 0 -section, which is compact. The boundedness with respect to some algebraic form is therefore implied by the boundedness of the volume of $\tilde{C} \cap \partial D(r)$ near $r=0$ when the volume is measured with respect to any particular Riemannian metric on the ambient manifold $T^{*} X$. Since we only need to establish boundedness of the volume, we can make several simplifying assumptions. First of all, the assumption that $\tilde{C}$ consists of a single component of multiplicity one. Secondly, we can uniformize $\tilde{C}$ ([Hn1], [Hn2], [BM, theorem 0.1]) by a real analytic map $f: N \rightarrow T^{*} X$, with $N$ a compact real analytic manifold of the same dimension as $\tilde{C}$, and $f(N)=\tilde{C}$. The pullback to $N$ of the metric on $T^{*} X$ is bounded by a metric on $N$, and $f^{-1}(\partial D(r))$ covers $\tilde{C} \cap \partial D(r)$ generically finitely. This leaves us with the following problem: a compact, connected, real analytic manifold $N$, a nonconstant real analytic function $r$, and we must show that the level sets $r=c$ have volume depending continuously on $c$. At noncritical values of $r$, this is obvious. To deal with critical values, one can put $r$ into normal form by a further step of uniformization ([Hn1], [Hn2], [BM, corollary 4.9]).

Lemmas 3.16 and 3.19 give us the right notion of restricted homology: we consider semi-algebraic cycles, on whose support $\operatorname{Re} \mu$ remains bounded, modulo boundaries of chains satisfying the same conditions. According to the two lemmas, the integral of $\mu_{\lambda}^{*} \hat{\phi}\left(-\sigma+\pi^{*} \tau_{\lambda}\right)^{n}$ over a restricted homology class becomes a well defined quantity. 


\section{CharaCter CyCles}

Invariant eigendistributions, either on $G_{\mathbb{R}}$ or on $\mathfrak{g}_{\mathbb{R}}$, are locally $L^{1}$ functions, real analytic on, respectively, $G_{\mathbb{R}}{ }^{\prime}$ and $\mathfrak{g}_{\mathbb{R}}{ }^{\prime}$, the sets of regular semisimple elements in $G_{\mathbb{R}}$ and $\mathfrak{g}_{\mathbb{R}}$ - that is, the assertion of Harish-Chandra's regularity theorem [HC4]. The complements of $G_{\mathbb{R}}{ }^{\prime}$ and $\mathfrak{g}_{\mathbb{R}}{ }^{\prime}$ have measure zero, so an invariant eigendistribution is completely determined by its restriction to the set of regular semisimple elements.

For definiteness, let us talk about the group case; at various points we shall indicate how statements need to be modified for the case of the Lie algebra. We choose a set of representatives $\left\{T_{1, \mathbb{R}}, \ldots, T_{m, \mathbb{R}}\right\}$ of the finitely many conjugacy classes of Cartan subgroups. Then the conjugates of the $G_{\mathbb{R}}{ }^{\prime} \cap T_{i, \mathbb{R}}, 1 \leq i \leq m$, cover $G_{\mathbb{R}}{ }^{\prime}$, so any invariant eigendistribution $\Theta$ is completely determined by its restriction to the various $G_{\mathbb{R}}{ }^{\prime} \cap T_{i, \mathbb{R}}$. To simplify the notation, we pick out one of the $T_{i, \mathbb{R}}$, drop the subscript $i$, and set $T_{\mathbb{R}}^{\prime}=G_{\mathbb{R}}{ }^{\prime} \cap T_{\mathbb{R}}$. The invariant eigendistribution $\Theta$ corresponds to an infinitesimal character $\chi_{\lambda}: \mathcal{Z}(\mathfrak{g}) \rightarrow \mathbb{C}$, with $\lambda \in \mathfrak{h}^{*}$. The parameter $\lambda$ is only determined up to Weyl conjugation, but we pick a specific representative. As we have done before, we index the various identifications between the universal Cartan $\mathfrak{h}$ and the complexified Lie algebra $\mathfrak{t}$ of $T_{\mathbb{R}}$ by the fixed points of $T_{\mathbb{R}}$ on the flag manifold $X$ :

$$
\mathfrak{t} \cong \mathfrak{b}_{x} /\left[\mathfrak{b}_{x}, \mathfrak{b}_{x}\right] \cong \mathfrak{h} \quad\left(x \in X^{T_{\mathbb{R}}}=\text { fixed point set of } T_{\mathbb{R}}\right) .
$$

These isomorphisms induce isomorphisms $\mathfrak{h}^{*} \cong \mathfrak{t}^{*}, \mu \mapsto \mu_{x}$. The universal Weyl group $W$ acts canonically and simply transitively on the fixed point set of $T_{\mathbb{R}}$. Initially we choose a particular fixed point $x$. Then, for any given $g \in T_{\mathbb{R}}^{\prime}$, there exist polynomial functions $p_{g, w}$ on $\mathfrak{t}$, indexed by $g$ and by $w \in W / W_{\lambda}$, the quotient of $W$ by the isotropy subgroup at $\lambda$, such that

$$
\Theta(g \exp \zeta)=\frac{\sum_{w \in W / W_{\lambda}} p_{g, w}(\zeta) e^{(w \lambda)_{x}(\zeta)-\rho_{x}(\zeta)}}{\prod_{\alpha \in \Phi^{+}}\left(1-e^{-\alpha_{x}}\right)(g \exp (\zeta))}
$$

for all small $\zeta \in \mathfrak{t}_{\mathbb{R}}$. Here $\Phi^{+}$and $\rho$ have the same meaning as in (2.10), and $e^{-\alpha_{x}}$ denotes the character of $T_{\mathbb{R}}$ corresponding to the root $-\alpha_{x}$, as is customary. These local expressions, even for only a single $g$ in every connected component of $G_{\mathbb{R}}{ }^{\prime} \cap T_{i, \mathbb{R}}$ and every $i$, completely determine $\Theta$. Conversely $\Theta$ determines the coefficient polynomials $p_{g, w}$, which have degree not exceeding the order of $W_{\lambda}$ - in particular, the $p_{g, w}$ are constant whenever $\lambda$ is regular.

The preceding statements constitute the easy part of Harish-Chandra's proof of the regularity theorem. One may ask whether a collection of local expressions as in (4.2) does define an invariant eigendistribution. For this it is certainly necessary that the local expressions fit together and give global, well defined functions on the various $G_{\mathbb{R}}{ }^{\prime} \cap T_{i, \mathbb{R}}$. Beyond this obvious necessary condition, there are necessary and sufficient conditions due to Harish-Chandra [HC4], usually referred to as the HarishChandra matching conditions. These involve the continuations of the numerators of the local expressions (4.2) to the subregular semisimple points; such points lie in (conjugates of) at most two of the $T_{i, \mathbb{R}}$, and the matching conditions relate the numerators on those Cartans.

The coefficient polynomials are constant not only when $\lambda$ is regular, as we had remarked earlier, but also when $\Theta$ is a virtual character corresponding to a possibly singular infinitesimal character; this observation was made by Fomin-Shapovalov [FS]. Conversely, an invariant eigendistribution $\Theta$ whose local expressions involve 
only constant coefficients is a $\mathbb{C}$-linear combination of characters. From now on we shall restrict attention to this particular situation: we assume that the $p_{g, w}$ are constants, as will be the case for all virtual characters.

Let us rewrite the local expression (4.2) in slightly more invariant terms. As before, $g \in T_{\mathbb{R}}$ will be regular, and $\zeta \in \mathfrak{t}_{\mathbb{R}}$ sufficiently small. We note that $(w \lambda)_{w x}=$ $\lambda_{x}$, hence

$$
\Theta(g \exp \zeta)=\sum_{x \in X^{T_{\mathbb{R}}}} \frac{c_{g, x} e^{\lambda_{x}(\zeta)-\rho_{x}(\zeta)}}{\prod_{\alpha \in \Phi^{+}}\left(1-e^{-\alpha_{x}}\right)(g \exp (\zeta))}
$$

summed over the fixed point set $X^{T_{\mathbb{R}}}$ of $T_{\mathbb{R}}$. Because of our assumption, the coefficients $c_{g, x}$ are constants. They are parametrized by $x \in X^{T_{\mathbb{R}}}$, rather than $w \in W / W_{\lambda}$ as in (4.2), so there will be repetitions in the sum when $\lambda$ is singular. Now, to make the $c_{g, x}$ unique, we require that $c_{g, v x}=\operatorname{sgn}(v) c_{g, x}$ for $v \in W_{\lambda}$ - the sign accounts for the fact that the fixed point $x$ has become variable whereas we had chosen a particular fixed point in (4.2).

The formula (4.3) has an analogue on the Lie algebra, which follows from (4.3) and the universal expression

$$
\left.\exp _{*}\right|_{\zeta}=\ell(\exp (\zeta))_{*} \circ \frac{1-e^{-\operatorname{ad} \zeta}}{\operatorname{ad} \zeta}
$$

for the differential of the exponential map. The analogue on the Lie algebra has a slightly simpler appearance, because roots and weights are globally well defined functions on the Cartan subalgebra $\mathfrak{t}_{\mathbb{R}}$ : there exist constants $d_{E, x}$, indexed by the various connected components $E \subset \mathfrak{t}_{\mathbb{R}}^{\prime}=\mathfrak{g}_{\mathbb{R}}{ }^{\prime} \cap \mathfrak{t}_{\mathbb{R}}$ and $x \in X^{T_{\mathbb{R}}}$, such that

$$
\theta(\zeta)=\sum_{x \in X^{T_{\mathbb{R}}}} \frac{d_{E, x} e^{\lambda_{x}}(\zeta)}{\prod_{\alpha \in \Phi^{+}} \alpha_{x}(\zeta)} \quad \text { for all } \zeta \in E \subset \mathfrak{t}_{\mathbb{R}}^{\prime} ;
$$

here $\theta$ and $\Theta$ are related by the formula (2.1). As in (4.3), the coefficients become uniquely determined when one requires $d_{E, v x}=\operatorname{sgn}(v) d_{E, x}$ for all $v \in W_{\lambda}$.

We return to the group case (4.3). Hotta-Kashiwara [HK], [Ka5] have given geometric meaning to the coefficients $c_{g, x}$. Let $\tilde{G}$ denote the variety of pairs $(g, x) \in$ $G \times X$, such that $x$ is a fixed point of $g$. When one makes $G$ act on itself by conjugation and on $X$ by translation, the two projections

$$
\pi: \tilde{G} \longrightarrow X, \quad q: \tilde{G} \longrightarrow G
$$

become $G$-equivariant. The former exhibits $\tilde{G}$ as the tautological bundle of Borel subgroups; in particular, $\tilde{G}$ is smooth. On the other hand, $q$ induces a (Galois) covering map over the regular set,

$$
\tilde{G}^{\prime}=q^{-1}\left(G^{\prime}\right) \stackrel{q}{\longrightarrow} G^{\prime}, \quad \text { with covering group } W,
$$

since $W$ acts simply transitively on the fixed point set $X^{g}$ of any $g \in G^{\prime}$. Note that

$$
\tilde{G_{\mathbb{R}}}=\left\{(g, x) \in \tilde{G} \mid g \in G_{\mathbb{R}}\right\}=q^{-1} G_{\mathbb{R}}
$$

is a - usually singular - real algebraic subvariety of $\tilde{G}$. Recall (2.4): since $\pi^{-1}(x)=$ $B_{x}$ for $x \in X$, there exists a natural map

$$
\tilde{G} \longrightarrow H
$$

from $\tilde{G}$ to the universal Cartan group $H$, which becomes $G$-equivariant when one thinks of $G$ as acting trivially on $H$. For $\lambda \in \mathfrak{h}$, the multiple valued function $e^{\lambda-\rho}$ 
generates a rank one local system on $H$, which entered the definition of the twisted $G_{\mathbb{R}}$-equivariant derived category $\mathrm{D}_{G_{\mathbb{R}}}(X)_{\lambda}$ in $\S 2$. The pullback, via (4.8), of this local system from $H$ to $\tilde{G}$ defines a $G$-equivariant rank one local system $\mathbb{C}_{\lambda}$ and a $G$-equivariant inclusion

$$
\mathbb{C}_{\lambda} \hookrightarrow \mathcal{O}_{\tilde{G}}
$$

into the sheaf of holomorphic functions. The local systems $\mathbb{C}_{\lambda}, \lambda \in \mathfrak{h}$, satisfy the periodicity condition analogous to (2.13): there exists a natural isomorphism

$$
\mathbb{C}_{\lambda} \cong \mathbb{C}_{\lambda+\mu} \quad \text { if } \mu \in \mathfrak{h}^{*} \text { is } H \text {-integral, }
$$

given by multiplication with the globally defined holomorphic function $e^{\mu}$ on $\tilde{G}$. As in (2.12), $\lambda=\rho$ corresponds to the "untwisted" case $\mathbb{C}_{\rho}=\mathbb{C}_{\tilde{G}}$.

Appearances to the contrary, the local formula (4.3) has global meaning: each regular $g \in G_{\mathbb{R}}{ }^{\prime}$ lies in a unique Cartan subgroup $T_{\mathbb{R}} \subset G_{\mathbb{R}}$, and $\Theta$ is conjugation invariant and real analytic on $G_{\mathbb{R}^{\prime}}$. It follows that the coefficients $c_{g, x}$, which are indexed by pairs $(g, x) \in \tilde{G}_{\mathbb{R}}{ }^{\prime}=q^{-1}\left(G_{\mathbb{R}}{ }^{\prime}\right)$, are $G_{\mathbb{R}}$-invariant, real analytic functions on ${\tilde{G_{\mathbb{R}}}}^{\prime}$. Setting $\zeta=0$ in (4.3) gives the global expression

$$
\Theta(g)=\sum_{x \in X^{g}} \frac{c_{g, x}}{\prod_{\alpha \in \Phi^{+}}\left(1-e^{-\alpha_{x}}\right)(g)} \quad\left(g \in G_{\mathbb{R}^{\prime}}{ }^{\prime} .\right.
$$

Again by (4.3), $c_{g, x} e^{(\lambda-\rho)_{x}(\zeta)}=c_{g} \exp \zeta, x$ whenever $\zeta \in \mathfrak{g}_{\mathbb{R}}$ is small and centralizes $g$. It follows that

$$
c:(g, x) \mapsto c_{g, x} \text { is a section of } \mathbb{C}_{\lambda} \subset \mathcal{O}_{\tilde{G}} \text { over }{\tilde{G_{\mathbb{R}}}}^{\prime} .
$$

We fix an orientation of $G_{\mathbb{R}}$; this induces an orientation also on $\tilde{G}_{\mathbb{R}}{ }^{\prime}$ via the covering map (4.6). Then, by Poincaré duality,

$$
c \in \mathrm{H}^{0}\left(\tilde{G_{\mathbb{R}}}{ }^{\prime}, \mathbb{C}_{\lambda}\right)=\mathrm{H}_{d}^{i n f}\left(\tilde{G_{\mathbb{R}}}{ }^{\prime}, \mathbb{C}_{-\lambda+2 \rho}\right) \cong \mathrm{H}_{d}^{i n f}\left(\tilde{G_{\mathbb{R}}}, \mathbb{C}_{-\lambda}\right) ;
$$

here $d=\operatorname{dim}_{\mathbb{R}} G_{\mathbb{R}}$ is the dimension of $\tilde{G_{\mathbb{R}}}$ and $\mathrm{H}_{*}^{\text {inf }}\left(\cdot, \mathbb{C}_{-\lambda}\right)$ denotes homology with locally finite support and values in the local system $\mathbb{C}_{-\lambda}$. The passage from $\mathbb{C}_{\lambda}$ to the dual local system $\mathbb{C}_{-\lambda+2 \rho}$ is forced by the formalism of Poincaré duality, since cohomology behaves covariantly with respect to the coefficient system, whereas homology behaves contravariantly. ${ }^{7}$

Homology with locally finite supports can be restricted to open subsets. Since $d$ is the dimension of $\tilde{G_{\mathbb{R}}}$,

$$
\mathrm{H}_{d}^{i n f}\left(\tilde{G_{\mathbb{R}}}, \mathbb{C}_{-\lambda}\right) \hookrightarrow \mathrm{H}_{d}^{i n f}\left({\tilde{G_{\mathbb{R}}}}^{\prime}, \mathbb{C}_{-\lambda}\right)
$$

is an inclusion. Hotta-Kashiwara [HK], [Ka5] show that every $c=c(\Theta)$, coming from an invariant eigendistribution $\Theta$ via (4.11)-(4.14), lies in $H_{d}^{i n f}\left(\tilde{G}_{\mathbb{R}}, \mathbb{C}_{-\lambda}\right)$; conversely, every $c \in \mathrm{H}_{d}^{\text {inf }}\left(\tilde{G_{\mathbb{R}}}, \mathbb{C}_{-\lambda}\right)$ determines an invariant eigendistribution. In effect, this is a reinterpretation of the Harish-Chandra matching conditions. Put differently, there exists a natural surjective map

$$
\mathrm{H}_{d}^{i n f}\left(\tilde{G_{\mathbb{R}}}, \mathbb{C}_{-\lambda}\right) \longrightarrow \mathcal{I E}_{c c}(\lambda)
$$

\footnotetext{
${ }^{7}$ More precisely, one can take cohomology with values in a sheaf, but homology with values in a cosheaf; local systems can be regarded as either, but the identification between the two is contravariant.
} 
onto $\mathcal{I E}_{c c}(\lambda)$, the space of invariant eigendistributions with constant coefficients $c_{g, w}$ and infinitesimal character $\chi_{\lambda}$. For regular $\lambda \in \mathfrak{h}^{*}$, this map is also injective. We had required earlier that the coefficients $c_{g, x}$ of any "character cycle" $c(\Theta)$ satisfy the symmetry condition

$$
c_{g, v x}=\operatorname{sgn}(v) c_{g, x} \quad \text { for } v \in W_{\lambda} .
$$

The map (4.15) becomes an isomorphism, even for singular $\lambda$, when restricted to the subspace of cycles $c$ subject to the symmetry condition (4.16).

We recall that we had fixed an orientation on $G_{\mathbb{R}}$ which affects the sign of the Poincaré duality map (4.13). Let us also fix a Haar measure $d g$ on $G_{\mathbb{R}}$. Together, these two choices determine a nonzero, $G_{\mathbb{R}}$-invariant form of top degree. Its complexification defines a $G$-invariant, holomorphic form $\omega$ on $G$, of top degree. The quotient map (4.8) allows us to view the $e^{\alpha}$, with $\alpha \in \Phi$, as holomorphic functions on $\tilde{G}$. The form $q^{*} \omega$ is divisible by the product of the $\left(1-e^{-\alpha}\right), \alpha \in \Phi^{+}-$see $[\mathrm{A}]$, for example - so

$$
\tilde{\omega}=\frac{q^{*} \omega}{\prod_{\alpha \in \Phi^{+}}\left(1-e^{-\alpha}\right)}
$$

exists as a well defined, $G$-invariant, holomorphic $d$-form on $\tilde{G}$. As such, it is locally integrable over any real algebraic $d$-chain in $\tilde{G}$. We make sense of the integral

$$
\int_{c}\left(q^{*} \phi\right) \tilde{\omega} \quad\left(c \in \mathrm{H}_{d}^{i n f}\left(\tilde{G_{\mathbb{R}}}, \mathbb{C}_{-\lambda}\right)\right),
$$

for any test function $\phi \in C_{c}^{\infty}\left(G_{\mathbb{R}}\right)$, by viewing $c$ as a collection of sections of $\mathbb{C}_{\lambda} \subset \mathcal{O}_{\tilde{G}}$ over the various connected components of $\tilde{G_{\mathbb{R}}}$. These sections are locally bounded on $\tilde{G}$. Thus we can multiply these sections and the integrand, and integrate the product over the various components of ${\tilde{G_{\mathbb{R}}}}^{\prime}$, using the orientation induced from $G_{\mathbb{R}}$. When $c$ corresponds to $\Theta \in \mathcal{I E}_{c c}(\lambda)$ via the homomorphism (4.15),

$$
\int_{G_{\mathbb{R}}} \Theta \phi d g=\int_{c}\left(q^{*} \phi\right) \tilde{\omega} .
$$

This identity amounts to a slight rephrasing of the correspondence (4.15) between cycles and invariant eigendistributions - see the proof of Proposition 6.2 below. Note that the form $\tilde{\omega}$ was normalized by the choice of the Haar measure $d g$.

The passage from the local formula (4.3) to the character cycle $c(\Theta)$ has a counterpart on the Lie algebra. In analogy to (4.5)-(4.8), we define $\tilde{\mathfrak{g}}$ as the set of pairs $(\zeta, x) \in \mathfrak{g} \times X$ such that $\zeta \in \mathfrak{b}_{x}$, projections $\pi: \tilde{\mathfrak{g}} \rightarrow X, q: \tilde{\mathfrak{g}} \rightarrow \mathfrak{g}, \tilde{\mathfrak{g}} \rightarrow \mathfrak{h}$, as well as $\tilde{\mathfrak{g}_{\mathbb{R}}}=q^{-1} \mathfrak{g}_{\mathbb{R}}$ and $\tilde{\mathfrak{g}_{\mathbb{R}}}{ }^{\prime}=q^{-1}\left(\mathfrak{g}_{\mathbb{R}}{ }^{\prime}\right)$. Note that $\tilde{\mathfrak{g}}$ and the quotient map $\tilde{\mathfrak{g}} \rightarrow \mathfrak{h}$ have come up already, in $\S 2$, where $\tilde{\mathfrak{g}}$ was denoted by $\mathcal{B}$; we are switching to different notation now to emphasize the analogy with the group case. The function $e^{\lambda-\rho}$, pulled back from $\mathfrak{h}$ to $\tilde{\mathfrak{g}}$, spans a rank one $\mathbb{C}$-subsheaf $\mathbb{C}_{\lambda} \subset \mathcal{O}_{\tilde{\mathfrak{g}}}$. Unlike in the group case, this sheaf is trivial as a sheaf of $\mathbb{C}$-vector spaces. The numerators $d_{E, x} e^{\lambda_{x}-\rho_{x}}$ in (4.4) determine a chain $c=c(\theta) \in \mathrm{H}_{d}^{\text {inf }}\left(\tilde{\mathfrak{g}}_{\mathbb{R}}{ }^{\prime}, \mathbb{C}_{-\lambda}\right)$, and the Harish-Chandra matching conditions on the Lie algebra imply that $c(\theta)$ is a cycle, i.e., $c(\theta) \in \mathrm{H}_{d}^{\text {inf }}\left(\tilde{\mathfrak{g}_{\mathbb{R}}}, \mathbb{C}_{-\lambda}\right)$. As before, we get a natural surjective map from $\mathrm{H}_{d}^{i n f}\left(\tilde{\mathfrak{g}_{\mathbb{R}}}, \mathbb{C}_{-\lambda}\right)$ to the vector space of invariant eigendistributions on $\mathfrak{g}_{\mathbb{R}}$, with constant local coefficients and with infinitesimal character $\chi_{\lambda}$. This map relates a cycle $c$ to the invariant eigendistribution 
$\theta$ via the formula

$$
\int_{\mathfrak{g}_{\mathbb{R}}} \theta \phi d \zeta=\int_{c}\left(q^{*} \phi\right) \tilde{\omega} \quad\left(\phi \in C_{c}^{\infty}\left(\mathfrak{g}_{\mathbb{R}}\right)\right) .
$$

Here $d \zeta$ is the Euclidean measure on $\mathfrak{g}_{\mathbb{R}}$, normalized so that $d \zeta$ and $\exp ^{*} d g$ coincide at the identity. Also, $\tilde{\omega}$ denotes the holomorphic $d$-form on $\tilde{\mathfrak{g}}$ obtained by complexifying the Euclidean measure $d \zeta$, pulling it back to $\tilde{\mathfrak{g}}$, and dividing by the product of the positive roots.

\section{The FIXED POINT FORMALISM AND Kashiwara's CONJECTURE}

The discussion in $\S 2$ attaches invariant eigendistributions $\Theta(\mathcal{F}), \theta(\mathcal{F})$ to objects $\mathcal{F} \in \mathrm{D}_{G_{\mathbb{R}}}(X)_{-\lambda}$. These, in turn, correspond to character cycles, in $\mathrm{H}_{d}^{\text {inf }}\left(\tilde{G}_{\mathbb{R}}, \mathbb{C}_{-\lambda}\right)$ and $\mathrm{H}_{d}^{\text {inf }}\left(\tilde{\mathrm{g}}, \mathbb{C}_{-\lambda}\right)$, respectively. Kashiwara [Ka4] has conjectured a direct geometric relationship between the sheaf $\mathcal{F}$ and the character cycle corresponding to $\Theta(\mathcal{F})$. This conjecture is equivalent, via (4.18), to an integral formula for the virtual character $\Theta(\mathcal{F})$, and also provides geometric expressions for the coefficients $c_{g, x}$ in the local formula (4.3) for $\Theta(\mathcal{F})$. When $G_{\mathbb{R}}$ happens to be compact, the local expressions for the $c_{g, x}$ reduce to the fixed point formula (1.5). There is one other situation in which characters had been calculated by means of a fixed point formula, prior to Kashiwara's conjecture: Hecht's formula for the characters of holomorphic discrete series representations [Hec], which also follows easily from the local fixed point formula presented here. In this section we recall Kashiwara's conjecture, which will be proved - along with its counterpart on the Lie algebra - in subsequent sections. We treat the case of a general infinitesimal character, which makes the discussion a bit heavy. Kashiwara only discusses the much more transparent case $\lambda=\rho$, so the reader may want to consult [Ka4] first.

The commuting actions of $G$ and the universal Cartan $H$ - see (2.9) - on the enhanced flag variety $\hat{X}$ induce an action map

$$
a: G_{\mathbb{R}} \times H \times \hat{X} \rightarrow \hat{X} .
$$

The projection onto the factor $\hat{X}$ defines a second morphism

$$
p: G_{\mathbb{R}} \times H \times \hat{X} \rightarrow \hat{X} .
$$

In analogy to the spaces $\tilde{G}, \tilde{G_{\mathbb{R}}}$, which were introduced in $\S 4$, we set

$$
\begin{aligned}
& \hat{G}=\{(g, h, \hat{x}) \in G \times H \times \hat{X} \mid a(g, h, \hat{x})=\hat{x}=p(g, h, \hat{x})\}, \\
& \hat{G_{\mathbb{R}}}=\hat{G} \cap\left(G_{\mathbb{R}} \times H \times \hat{X}\right) .
\end{aligned}
$$

Note that there is a natural $H$-fibration $\hat{G} \rightarrow \tilde{G}$, obtained by eliminating the $H$ factor in the product $G \times H \times \hat{X}$ and mapping $\hat{X}$ to $X$. Composing this fibration with the natural map (4.8) results in a morphism $\tau: \hat{G} \rightarrow H$. We claim: the diagram

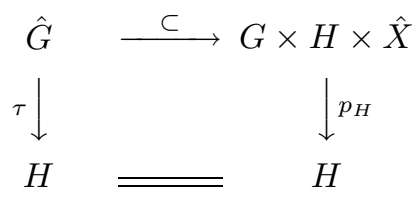


commutes. It suffices to check this over any given point $x \in X$. We can identify $\hat{X}$ with $G / N_{x}$ and $H$ with $B_{x} / N_{x}$; with these identifications, the verification is straightforward.

The rank one local system $\mathbb{C}_{\lambda}$ on $\tilde{G}$ was defined as the pullback of a local system on $H$ via the projection (4.8) - the local system generated by the multiple valued function $e^{\lambda-\rho}$. The commutativity of the diagram (5.3) allows us to think of $\mathbb{C}_{\lambda}$ as a "universal" rank one local system also on the spaces $\hat{G}$ and $G \times H \times \hat{X}$. We shall use the same symbol $\mathbb{C}_{\lambda}$ in all cases, to avoid complicated notation. Note that $\mathbb{C}_{\lambda}$ is canonically a subsheaf of the sheaf of holomorphic functions on the various spaces, in analogy to (4.9), compatibly with the natural morphisms between the spaces.

We recall the definition, in $\S 2$, of the twisted $G_{\mathbb{R}^{-}}$equivariant derived category $\mathrm{D}_{G_{\mathbb{R}}}(X)_{\lambda}$. Disregarding part of the structure, we get a "forgetful functor"

$$
\mathrm{D}_{G_{\mathbb{R}}}(X)_{\lambda} \longrightarrow \mathrm{D}^{b}\left(S h_{X, \lambda}\right)
$$

into the bounded derived category of $(\lambda-\rho)$-monodromic sheaves on $X$. The latter derived category is a full subcategory of $\mathrm{D}^{b}(\hat{X})$,

$$
\mathrm{D}^{b}\left(S h_{X, \lambda}\right) \hookrightarrow \mathrm{D}^{b}(\hat{X}) .
$$

The composition of $(5.1 \mathrm{a}, \mathrm{b})$ gives a functor

$$
\mathrm{D}_{G_{\mathbb{R}}}(X)_{\lambda} \longrightarrow \mathrm{D}^{b}(\hat{X}), \quad \mathcal{G} \mapsto \hat{\mathcal{G}} .
$$

Recall the definitions (5.1). We claim: for each $\mathcal{G} \in \mathrm{D}_{G_{\mathbb{R}}}(X)_{\lambda}$, there exists a canonical morphism

$$
\phi: a^{*} \hat{\mathcal{G}} \rightarrow p^{*} \hat{\mathcal{G}} \otimes \mathbb{C}_{\lambda}
$$

in $\mathrm{D}^{b}\left(G_{\mathbb{R}} \times H \times \hat{X}\right)$, which will play a crucial role in the fixed point formalism.

To construct $\phi$, we consider the diagram

$$
G_{\mathbb{R}} \times \mathfrak{h} \times \hat{X} \stackrel{\epsilon}{\longrightarrow} G_{\mathbb{R}} \times H \times \hat{X} \stackrel{a, p}{\longrightarrow} \hat{X},
$$

where $\epsilon(g, \zeta, \hat{x})=(g, \exp (\zeta), \hat{x})$. Note that the compositions $\epsilon a, \epsilon p$ are the action and projection morphisms of the $\left(G_{\mathbb{R}} \times \mathfrak{h}\right)$-action on $\hat{X}$. By definition, $\mathrm{D}_{G_{\mathbb{R}}}(X)_{\lambda}$ is a full subcategory of the $\left(G_{\mathbb{R}} \times \mathfrak{h}\right)$-equivariant derived category on $\hat{X}$. The formalism of this latter equivariant derived category implies the existence of a distinguished isomorphism

$$
\phi^{\prime}: \epsilon^{*} a^{*} \hat{\mathcal{G}} \rightarrow \epsilon^{*} p^{*} \hat{\mathcal{G}} .
$$

The single isomorphism $\phi^{\prime}$ encodes a family of isomorphisms

$$
\begin{aligned}
& \phi_{g, \zeta}^{\prime}: \ell_{g, \zeta}^{*} \hat{\mathcal{G}} \stackrel{\sim}{\rightarrow} \hat{\mathcal{G}} \quad\left(\ell_{g, \zeta}=\text { translation by }(g, \zeta) \in G_{\mathbb{R}} \times \mathfrak{h}\right), \\
& \phi_{g, \zeta}^{\prime}=\text { restriction of } \phi^{\prime} \text { to }\{g\} \times\{\zeta\} \times \hat{X},
\end{aligned}
$$

which depend multiplicatively and continuously on the parameters. Since $\mathfrak{h}$ is simply connected, we may view $e^{\lambda-\rho}$ as a well-defined section of $\epsilon^{*} \mathbb{C}_{\lambda}$. We now define

$$
\phi^{\prime \prime}: \epsilon^{*} a^{*} \hat{\mathcal{G}} \rightarrow \epsilon^{*} p^{*} \hat{\mathcal{G}} \otimes \epsilon^{*} \mathbb{C}_{\lambda}
$$


by the formula $\phi^{\prime \prime}=e^{\lambda-\rho} \phi^{\prime}$. Any $\zeta \in \mathfrak{h}_{\mathbb{Z}}={ }_{\text {def }} \operatorname{Ker}(\mathfrak{h} \rightarrow H)$ acts on $\hat{X}$ as translation by $\exp (\zeta)=1$. The monodromicity condition in the definition of $S h_{X, \lambda}$ implies:

$$
\phi_{e, \zeta}^{\prime}: \ell_{e, \zeta}^{*} \hat{\mathcal{G}}=\hat{\mathcal{G}} \rightarrow \hat{\mathcal{G}} \text { is multiplication by } e^{-(\lambda-\rho)(\zeta)},
$$

for $\zeta \in \mathfrak{h}_{\mathbb{Z}}$, as before. It follows that $\phi^{\prime \prime}$ is invariant under translation by the lattice $\mathfrak{h}_{\mathbb{Z}}$, and therefore drops to an isomorphism $a^{*} \hat{\mathcal{G}} \stackrel{\sim}{\rightarrow} p^{*} \hat{\mathcal{G}} \otimes \mathbb{C}_{\lambda}$. That is, the isomorphism $\phi$ whose existence we postulated earlier.

Two comments are in order. The statement (5.8) amounts to a reinterpretation of the monodromicity condition in the language of the equivariant derived category. Secondly, the category $D_{G_{\mathbb{R}}}(X)_{\lambda}$ depends only on the image of $\lambda$ in $\mathfrak{h}^{*} / \Lambda$, where $\Lambda$ denotes the weight lattice, i.e., the lattice dual to $(2 \pi i)^{-1} \mathfrak{h}_{\mathbb{Z}}$. The definition of $\phi$, on the other hand, involves the section $e^{\lambda-\rho}$ of $\epsilon^{*} \mathbb{C}_{\lambda}$, and thus depends on $\lambda$ itself.

To each $\mathcal{G} \in \mathrm{D}_{G_{\mathbb{R}}}(X)_{\lambda}$, we associate a cycle in $\mathrm{H}_{d}^{\text {inf }}\left(\tilde{G}_{\mathbb{R}}, \mathbb{C}_{-\lambda}\right)$, of degree $d=$ $\operatorname{dim}\left(G_{\mathbb{R}}\right)$, as follows. We consider the Cartesian diagram

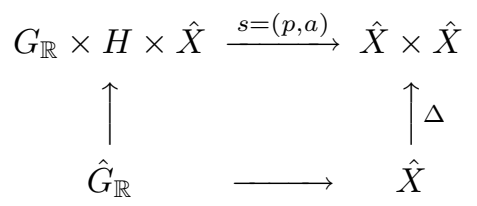

where $\Delta$ is the diagonal map. Consider the following sequence of maps:

$$
\begin{aligned}
\operatorname{Hom}(\hat{\mathcal{G}}, \hat{\mathcal{G}}) \stackrel{\sim}{\rightarrow} \mathrm{H}_{\Delta \hat{X}}^{0}(\hat{X} \times \hat{X}, \mathbb{D} \hat{\mathcal{G}} \otimes \hat{\mathcal{G}}) \\
\stackrel{s^{*}}{\rightarrow} \mathrm{H}_{\hat{G}_{\mathbb{R}}}^{0}\left(G_{\mathbb{R}} \times H \times \hat{X}, s^{*}(\mathbb{D} \hat{\mathcal{G}} \otimes \hat{\mathcal{G}})\right) \\
\stackrel{\sim}{\rightarrow} \mathrm{H}_{\hat{G}_{\mathbb{R}}}^{0}\left(G_{\mathbb{R}} \times H \times \hat{X}, p^{*} \mathbb{D} \hat{\mathcal{G}} \otimes a^{*} \hat{\mathcal{G}}\right) \\
\stackrel{\phi}{\rightarrow} \mathrm{H}_{\hat{G}_{\mathbb{R}}}^{0}\left(G_{\mathbb{R}} \times H \times \hat{X}, p^{*} \mathbb{D} \hat{\mathcal{G}} \otimes p^{*} \hat{\mathcal{G}} \otimes \mathbb{C}_{\lambda}\right) \\
\stackrel{\eta}{\rightarrow} \mathrm{H}_{\hat{G}_{\mathbb{R}}}^{0}\left(G_{\mathbb{R}} \times H \times \hat{X}, p^{*} \mathbb{D}_{\hat{X}} \otimes \mathbb{C}_{\lambda}\right) \\
\quad \stackrel{\sim}{\rightarrow} \mathrm{H}_{d+r}^{\text {inf }}\left(\hat{G}_{\mathbb{R}}, \mathbb{C}_{-\lambda}\right) \stackrel{\sim}{\rightarrow} \mathrm{H}_{d}^{\text {inf }}\left(\tilde{G}_{\mathbb{R}}, \mathbb{C}_{-\lambda}\right),
\end{aligned}
$$

with $r=\operatorname{dim}_{\mathbb{R}}(H)$. We use our previous notational conventions: $\mathrm{H}_{Z}^{p}(\ldots)$ denotes local cohomology along $Z, \mathrm{H}_{p}^{\text {inf }}(\ldots)$ is homology with infinite supports, and $d=$ $\operatorname{dim}\left(G_{\mathbb{R}}\right)$. However, we define the Verdier dual $\mathbb{D} \hat{\mathcal{G}}$ of $\hat{\mathcal{G}}$ using the dualizing sheaf $\mathbb{D}_{\hat{X}}$ on $\hat{X}$; this differs formally from our definition in $\S 2$, but does not effect the results to be proved, as pointed out in footnote 4 in $\S 2$.

Let us explain the various steps in (5.10). The isomorphism on the first line follows from the constructibility of $\hat{\mathcal{G}}$ - see, for example, propositions 3.1 .14 and 3.4.4 in [KSa]. The morphism $s^{*}$ is the pullback of local cohomology, and the next isomorphism follows from the interpretation of the external tensor product $\nabla$ in terms of the usual (internal) tensor product of sheaves. The morphism $\eta$ is induced by the duality pairing $\mathbb{D} \hat{\mathcal{G}} \otimes \hat{\mathcal{G}} \rightarrow \mathbb{D}$. On the most naive level, the next to last isomorphism can be understood by identifying $p^{*} \mathbb{D}_{\hat{X}}$ with the constant sheaf, appropriately shifted, and then applying Poincare duality; recall the reason for the passage from $\mathbb{C}_{\lambda}$ to $\mathbb{C}_{-\lambda}$ as explained in $\S 4$. More formally,

$$
p^{*} \mathbb{D}_{\hat{X}} \simeq p^{!} \mathbb{D}_{\hat{X}}[-(d+r)] \simeq \mathbb{D}_{G_{\mathbb{R}} \times H \times \hat{X}}[-(d+r)],
$$


hence

$$
\begin{aligned}
p^{*} \mathbb{D}_{\hat{X}} \otimes \mathbb{C}_{\lambda} \simeq\left(\mathbb{D}_{G_{\mathbb{R}} \times H \times \hat{X}} \otimes \mathbb{C}_{\lambda}\right)[-(d+r)] & \\
& \simeq R \mathcal{H o m}\left(\mathbb{C}_{-\lambda}, \mathbb{D}_{G_{\mathbb{R}} \times H \times \hat{X}}\right)[-(d+r)] \quad\left(\mathbb{C}_{-\lambda} \text { is locally free! }\right) \\
& ={ }_{\text {def }} \mathbb{D}\left(\mathbb{C}_{-\lambda}\right)[-(d+r)] .
\end{aligned}
$$

Let $i$ denote the inclusion $\hat{G}_{\mathbb{R}} \subset G_{\mathbb{R}} \times H \times \hat{X}$; then

$$
\begin{aligned}
& \mathrm{H}_{\hat{G}_{\mathbb{R}}}^{0}\left(G_{\mathbb{R}} \times H \times \hat{X}, p^{*} \mathbb{D}_{\hat{X}} \otimes \mathbb{C}_{\lambda}\right) \simeq \mathrm{H}_{\hat{G}_{\mathbb{R}}}^{-d-r}\left(G_{\mathbb{R}} \times H \times \hat{X}, \mathbb{D}\left(\mathbb{C}_{-\lambda}\right)\right) \\
& \simeq \mathrm{H}^{-d-r}\left(\hat{G}_{\mathbb{R}}, i^{!} \mathbb{D}\left(\mathbb{C}_{-\lambda}\right)\right) \quad \text { (by definition of local cohomology) } \\
& \simeq \mathrm{H}^{-d-r}\left(\hat{G_{\mathbb{R}}}, \mathbb{D}\left(i^{*} \mathbb{C}_{-\lambda}\right)\right) \\
& =\mathrm{H}^{-d-r}\left(\hat{G_{\mathbb{R}}}, \mathbb{D}\left(\mathbb{C}_{-\lambda}\right)\right) \quad \text { (according to our notational conventions) } \\
& \simeq \mathrm{H}_{d+r}^{i n f}\left(\hat{G}_{\mathbb{R}}, \mathbb{C}_{-\lambda}\right) \quad \text { (by definition of homology). }
\end{aligned}
$$

The last isomorphism in (5.10) follows from two facts: we are dealing with homology (with locally finite support) in top degree, and $\hat{G_{\mathbb{R}}} \rightarrow \tilde{G_{\mathbb{R}}}$ is a fibration with $r$ dimensional fibers. The symbol $\mathbb{C}_{\lambda}$, we recall, refers to sheaves on the various spaces, all coming by pull back from the same local system on $H$, so $\mathbb{C}_{\lambda}$ on $\hat{G}_{\mathbb{R}}$ is the pullback of $\mathbb{C}_{\lambda}$ on $\tilde{G}_{\mathbb{R}}$.

We can assign a cycle in $\mathrm{H}_{d}^{\text {inf }}\left(\tilde{G}_{\mathbb{R}}, \mathbb{C}_{-\lambda}\right)$ to any given $\mathcal{G} \in \mathrm{D}_{G_{\mathbb{R}}}(X)_{\lambda}$ by taking the image of $1_{\hat{\mathcal{G}}} \in \operatorname{Hom}(\hat{\mathcal{G}}, \hat{\mathcal{G}})$ under the sequence of maps $(5.10)$. As a matter of notation, we write

$$
c(\mathcal{F}) \in \mathrm{H}_{d}^{\text {inf }}\left(\tilde{G}_{\mathbb{R}}, \mathbb{C}_{-\lambda}\right) \quad\left(\mathcal{F} \in \mathrm{D}_{G_{\mathbb{R}}}(X)_{-\lambda}\right)
$$

for the cycle attached to $\mathcal{G}=\mathbb{D} \mathcal{F}$. Recall $(2.14)$ : since the definition of $D_{G_{\mathbb{R}}}(X)_{\lambda}$ involves the monodromy behavior of $e^{(\lambda-\rho)}$, Verdier duality maps $D_{G_{\mathbb{R}}}(X)_{-\lambda}$ to $D_{G_{\mathbb{R}}}(X)_{\lambda+2 \rho}$; on the other hand, $D_{G_{\mathbb{R}}}(X)_{\lambda+2 \rho}=D_{G_{\mathbb{R}}}(X)_{\lambda}$ since $2 \rho$ is integral, so $\mathbb{D}: D_{G_{\mathbb{R}}}(X)_{-\lambda} \rightarrow D_{G_{\mathbb{R}}}(X)_{\lambda}$.

5.12 Theorem. The cycle $c(\mathcal{F})$, for $\mathcal{F} \in \mathrm{D}_{G_{\mathbb{R}}}(X)_{-\lambda}$, is the character cycle of the virtual character $\Theta(\mathcal{F})$. In particular,

$$
\int_{G_{\mathbb{R}}} \Theta(\mathcal{F}) \phi d g=\int_{c(\mathcal{F})}\left(q^{*} \phi\right) \tilde{\omega}
$$

for any test function $\phi \in C_{c}^{\infty}\left(G_{\mathbb{R}}\right)$.

This statement was conjectured by Kashiwara [Ka4]. We shall prove it in the later sections. We remind the reader that the homomorphism (4.15) is not injective, in general. However, it has a distinguished left inverse, namely the assignment $\Theta \mapsto$ character cycle of $\Theta$ - the character cycle, by definition, satisfies the symmetry condition (4.16). Our theorem asserts, in particular, that $c(\mathcal{F})$ satisfies this symmetry condition.

The theorem - more precisely, the construction preceding the theorem - can be simplified whenever $\lambda$ is integral. In this situation, the twisted equivariant derived category $\mathrm{D}_{G_{\mathbb{R}}}(X)_{-\lambda}$ agrees with the usual equivariant derived category $\mathrm{D}_{G_{\mathbb{R}}}(X)$ (recall (2.12)-(2.13)). Also, the "universal" local system $\mathbb{C}_{\lambda}$ has a distinguished, globally defined generating section $e^{\lambda-\rho}$. The cycle $c(\mathcal{F}) \in \mathrm{H}_{d}^{\text {inf }}\left(\tilde{G}_{\mathbb{R}}, \mathbb{C}_{-\lambda}\right)$ can now be written as a product of the generating section $e^{\lambda-\rho}$ with the absolute cycle $c\left(\mathcal{F}_{\rho}\right) \in \mathrm{H}_{d}^{\text {inf }}\left(\tilde{G}_{\mathbb{R}}, \mathbb{C}\right)$, where $\mathcal{F}_{\rho} \in \mathrm{D}_{G_{\mathbb{R}}}(X)$ is the image of $\mathcal{F}$ under the periodicity isomorphism (2.12)-(2.13). This allows us to work on $G_{\mathbb{R}} \times X$, rather than 
$G_{\mathbb{R}} \times H \times \hat{X}$ as before. In particular, the existence of the canonical isomorphism $\phi: a^{*} \mathcal{G} \rightarrow p^{*} \mathcal{G}$ immediately follows from the definition of the equivariant derived category, so (5.6)-(5.8) become superfluous, and (5.9)-(5.10) simplify correspondingly. Kashiwara's conjecture [Ka4] is phrased in these terms.

The proof of Theorem 5.12 depends on a local fixed point formula for the coefficients $c_{g, x}$ in the local expression (4.3) for $\Theta=\Theta(\mathcal{F})$. This local formula is of independent interest. Kashiwara develops a fixed point formalism in [Ka4] and uses it to calculate the cycle (5.11) from the geometric datum of the sheaf $\mathcal{F}$.

To state the local formula, we fix a regular semisimple $g \in G_{\mathbb{R}}^{\prime}$ and a fixed point $x \in X$ of $g$. The centralizer of $g$ in $G_{\mathbb{R}}$ is a Cartan subgroup $T_{\mathbb{R}}$, whose Lie algebra we denote by $\mathfrak{t}_{\mathbb{R}} ; \mathfrak{t}$ is the complexification of $\mathfrak{t}_{\mathbb{R}}$. The identification $\mathfrak{t} \cong \mathfrak{b}_{x} /\left[\mathfrak{b}_{x}, \mathfrak{b}_{x}\right] \cong \mathfrak{h}$ lifts to a natural identification

$$
T \cong B_{x} /\left[B_{x}, B_{x}\right] \cong H .
$$

It induces an identification $\alpha \mapsto \alpha_{x}$ between the universal root system $\Phi$ and the concrete root system $\Phi(\mathfrak{g}, \mathfrak{t})$. The complexified Cartan subgroup $T$ normalizes the Borel subalgebra

$$
\mathfrak{b}_{x}=\mathfrak{t} \oplus\left(\oplus_{\alpha \in \Phi^{+}} \mathfrak{g}^{-\alpha_{x}}\right) .
$$

Here $\mathfrak{g}^{\alpha_{x}}$, for $\alpha \in \Phi$, denotes the $\alpha_{x}$-root space of $(\mathfrak{g}, \mathfrak{t})$. Let

$$
\mathfrak{n}^{+}(g, x)=\oplus_{\alpha \in \Phi^{+}} \mathfrak{g}^{\alpha_{x}}
$$

denote the nilpotent radical of the opposite Borel, and let $\mathfrak{n}^{\prime}(g, x), \mathfrak{n}^{\prime \prime}(g, x)$ be two subalgebras of $\mathfrak{n}^{+}(g, x)$, of the following type. We choose subsets $\Psi^{\prime}, \Psi^{\prime \prime} \subset \Phi^{+}$, such that

$$
\begin{aligned}
& \text { a) for } \alpha \in \Phi^{+} \text {, if }\left|e^{\alpha_{x}}(g)\right| \neq 1 \text {, then } \\
& \alpha \in \Psi^{\prime} \Longleftrightarrow\left|e^{\alpha_{x}}(g)\right|<1 \text { and } \alpha \in \Psi^{\prime \prime} \Longleftrightarrow\left|e^{\alpha_{x}}(g)\right|>1 \text {; } \\
& \text { b) for } \alpha_{1}, \alpha_{2} \in \Phi^{+}, \text {if } \alpha_{1}+\alpha_{2} \in \Phi^{+} \text {, then } \\
& \alpha_{1}, \alpha_{2} \in \Psi^{\prime} \Longrightarrow \alpha_{1}+\alpha_{2} \in \Psi^{\prime}, \quad \alpha_{1}, \alpha_{2} \in \Psi^{\prime \prime} \Longrightarrow \alpha_{1}+\alpha_{2} \in \Psi^{\prime \prime} .
\end{aligned}
$$

Subsets $\Psi^{\prime}, \Psi^{\prime \prime}$ satisfying these conditions do exist: for example, the subsets defined by a) without the restriction $\left|e^{\alpha_{x}}(g)\right| \neq 1$ satisfy also b). Because of $\mathrm{b}$ ),

$$
\mathfrak{n}^{\prime}(g, x)=\oplus_{\alpha \in \Psi^{\prime}} \mathfrak{g}^{\alpha_{x}}, \quad \mathfrak{n}^{\prime \prime}(g, x)=\oplus_{\alpha \in \Psi^{\prime \prime}} \mathfrak{g}^{\alpha_{x}}
$$

are subalgebras of $\mathfrak{n}^{+}(g, x)$. Our eventual statement, Theorem 5.24, will not depend on the particular choice of $\Psi$. Indeed, the theorem is valid even with more general choices of $\mathfrak{n}^{\prime}(g, x)$ and $\mathfrak{n}^{\prime \prime}(g, x)$ - see the comment at the end of this section. Further notation:

$$
\begin{gathered}
N^{+}(g, x)=\exp \left(\mathfrak{n}^{+}(g, x)\right) x, \\
N^{\prime}(g, x)=\exp \left(\mathfrak{n}^{\prime}(g, x)\right) x, \quad N^{\prime \prime}(g, x)=\exp \left(\mathfrak{n}^{\prime \prime}(g, x)\right) x .
\end{gathered}
$$

Then $N^{+}(g, x) \subset X$ is an open Schubert cell which contains $N^{\prime}(g, x)$ and $N^{\prime \prime}(g, x)$ as affine linear subspaces, both invariant under the action of $T$.

The inverse image of $N^{+}(g, x)$ in $\hat{X}$ splits into a product $N^{+}(g, x) \times H$, and the $G \times H$-action on $\hat{X}$ restricts to a $T \times H$-action on $N^{+}(g, x) \times H$ :

$$
(t, h):\left(\exp (\zeta) x, h_{1}\right) \mapsto\left(\exp ((\operatorname{Ad} t)(\zeta)) x, t h_{1} h^{-1}\right), \quad \zeta \in \mathfrak{n}^{+}(g, x) ;
$$


here we identify $t \in T$ with an element of the universal Cartan $H$ via the isomorphism (5.13). In particular, there is a natural restriction functor

$$
\mathrm{D}_{G_{\mathbb{R}}}(X)_{\lambda} \rightarrow \mathrm{D}_{T_{\mathbb{R}}}\left(N^{+}(g, x)\right)_{\lambda},
$$

from the twisted equivariant derived category $\mathrm{D}_{G_{\mathbb{R}}}(X)_{\lambda}$, viewed as the $G_{\mathbb{R}}$-equivariant derived category of $(-\lambda-\rho)$-monodromic sheaves on $\hat{X}$, to the twisted equivariant derived category $\mathrm{D}_{T_{\mathbb{R}}}\left(N^{+}(g, x)\right)_{\lambda}$, viewed as the $T_{\mathbb{R}}$-equivariant derived category of $(-\lambda-\rho)$-monodromic sheaves on $N^{+}(g, x) \times H$. The base of the bundle $N^{+}(g, x) \times H \rightarrow N^{+}(g, x)$ is contractible. It follows that there is a canonical equivalence between the category of $(\lambda-\rho)$-monodromic sheaves on $N^{+}(g, x)$ on the one hand, and the category of (ordinary) sheaves on $N^{+}(g, x)$ on the other,

$$
\mathrm{D}_{T_{\mathbb{R}}}\left(N^{+}(g, x)\right)_{\lambda} \stackrel{\sim}{\rightarrow} \mathrm{D}_{T_{\mathbb{R}}}\left(N^{+}(g, x)\right) ;
$$

in one direction, sheaves on $N^{+}(g, x)$ can be pulled back to $N^{+}(g, x) \times H$ and tensored with the universal local system $\mathbb{C}_{\lambda}$, and in the opposite direction, twisted sheaves on $N^{+}(g, x) \times H$ can be restricted to $N^{+}(g, x) \times\{e\} \cong N^{+}(g, x)$. Thus we get a restriction functor

$$
\mathrm{D}_{G_{\mathbb{R}}}(X)_{\lambda} \rightarrow \mathrm{D}_{T_{\mathbb{R}}}\left(N^{+}(g, x)\right), \quad \mathcal{G} \mapsto \mathcal{G}(x),
$$

the composition of the functors $(5.19 \mathrm{a}, \mathrm{b})$.

The action and projection maps $a, p: G_{\mathbb{R}} \times H \times \hat{X} \rightarrow \hat{X}$ restrict to maps from $T_{\mathbb{R}} \times H \times N^{+}(g, x) \times H$ to $N^{+}(g, x) \times H$, to which we refer by the same letters $a, p$. We then get a commutative diagram

$$
\begin{array}{crr}
T_{\mathbb{R}} \times H \times N^{+}(g, x) \times H & \stackrel{a, p}{\longrightarrow} N^{+}(g, x) \times H \\
(t, y) \mapsto(t, t, y, e) & \uparrow y \mapsto(y, e) \\
T_{\mathbb{R}} \times N^{+}(g, x) & \stackrel{a, p}{\longrightarrow} & N^{+}(g, x) ;
\end{array}
$$

note that the action map in the top row is the action (5.18), and that the universal local system $\mathbb{C}_{\lambda}$ "lives" on the first of the two factors $H$ in $T_{\mathbb{R}} \times H \times N^{+}(g, x) \times H$. The morphism (5.5), which came from the structure of the twisted equivariant derived category, restricts to a morphism $\phi: a^{*} \mathcal{G} \rightarrow p^{*} \mathcal{G} \otimes \mathbb{C}_{\lambda}$ in the derived category $\mathrm{D}^{b}\left(T_{\mathbb{R}} \times H \times N^{+}(g, x) \times H\right)$, for each $\mathcal{G} \in \mathrm{D}_{G_{\mathbb{R}}}(X)_{\lambda}$. In (5.5), we wrote $\hat{\mathcal{G}}$ to signify that we were disregarding the equivariance. To be consistent, we might use the same convention now; however, to avoid proliferating notation, we shall not notationally distinguish between $\mathcal{G}$ and $\hat{\mathcal{G}}$ from now on. Via the commutative diagram (5.20), our present incarnation of $\phi$ induces a morphism

$$
\phi: a^{*} \mathcal{G}(x) \longrightarrow p^{*} \mathcal{G}(x) \otimes \mathbb{C}_{\lambda}
$$

in the category $\mathrm{D}^{b}\left(T_{\mathbb{R}} \times N^{+}(g, x)\right)$. In the statement that follows, we think of $x$ as fixed and $g$ as variable within $T_{\mathbb{R}}^{\prime}$. To emphasize the point, we shall denote this variable element as $t$. Note that the subspaces $N^{\prime}(t, x), N^{\prime \prime}(t, x)$, defined in analogy to $N^{\prime}(g, x)$ and $N^{\prime \prime}(g, x)$, depend only on the connected component of $T_{\mathbb{R}}^{\prime}$ in which $t$ lies, whereas $N^{+}(t, x)=N^{+}(g, x)$ for all $t \in T_{\mathbb{R}}^{\prime}$.

To keep the notation simple, we denote the restriction of the morphism (5.21) to the slice $\{t\} \times N^{+}(t, x)$ by the same letter $\phi$. This gives us a morphism

$$
\phi: t^{*} \mathcal{G}(x) \longrightarrow \mathcal{G}(x) \otimes \mathbb{C}_{\lambda} .
$$


The diffeomorphism $t: N^{+}(t, x) \rightarrow N^{+}(t, x)$ preserves the subspaces $N^{\prime}(t, x)$, $N^{\prime \prime}(t, x)$. Hence $\phi$ induces morphisms

$$
\begin{aligned}
& \phi: t^{*} \mathcal{H}_{N^{\prime}(t, x)}(\mathcal{G}(x)) \longrightarrow \mathcal{H}_{N^{\prime}(t, x)}(\mathcal{G}(x)) \otimes \mathbb{C}_{\lambda}, \\
& \phi: t^{*}\left(\left.\mathcal{G}(x)\right|_{N^{\prime \prime}(t, x)}\right) \longrightarrow\left(\left.\mathcal{G}(x)\right|_{N^{\prime \prime}(t, x)}\right) \otimes \mathbb{C}_{\lambda} .
\end{aligned}
$$

Since $x$ is a fixed point of $t$, (5.23a) induces morphisms of stalks, respectively costalks - i.e., local cohomology at a point -

$$
\begin{aligned}
\phi_{t} & : \mathcal{H}_{N^{\prime}(t, x)}(\mathcal{G}(x))_{x} \longrightarrow \mathcal{H}_{N^{\prime}(t, x)}(\mathcal{G}(x))_{x} \otimes \mathbb{C}_{\lambda}, \\
\phi_{t} & : \mathcal{H}_{\{x\}}\left(\left.\mathcal{G}(x)\right|_{N^{\prime \prime}(t, x)}\right) \longrightarrow \mathcal{H}_{\{x\}}\left(\left.\mathcal{G}(x)\right|_{N^{\prime \prime}(t, x)}\right) \otimes \mathbb{C}_{\lambda} .
\end{aligned}
$$

We can now state the local fixed point theorem, which gives us two separate, formally dual expressions for the section $c_{t, x}$ of $\mathbb{C}_{\lambda}$ appearing in the expression (4.11) of the virtual character $\Theta=\Theta(\mathcal{F})$ :

5.24 Theorem. For $\mathcal{F} \in \mathrm{D}_{G_{\mathbb{R}}}(X)_{-\lambda}$ and $t \in T_{\mathbb{R}}^{\prime}$, the section $c_{t, x}$ of $\mathbb{C}_{\lambda}$ satisfies

$$
\begin{aligned}
c_{t, x} & =\sum(-1)^{i} \operatorname{tr}\left(\phi_{t}: \mathcal{H}_{N^{\prime}(t, x)}^{i}(\mathbb{D} \mathcal{F}(x))_{x} \rightarrow \mathcal{H}_{N^{\prime}(t, x)}^{i}(\mathbb{D} \mathcal{F}(x))_{x} \otimes \mathbb{C}_{\lambda}\right) \\
& =\sum(-1)^{i} \operatorname{tr}\left(\phi_{t}: \mathcal{H}_{\{x\}}^{i}\left(\left.(\mathbb{D} \mathcal{F}(x))\right|_{N^{\prime \prime}(t, x)}\right) \rightarrow \mathcal{H}_{\{x\}}^{i}\left(\left.(\mathbb{D} \mathcal{F}(x))\right|_{N^{\prime \prime}(t, x)}\right) \otimes \mathbb{C}_{\lambda}\right) .
\end{aligned}
$$

Note that the presence of the factors $\mathbb{C}_{\lambda}$ makes the traces into sections, as they should be, rather than scalars.

Let us suppose, for the moment, that a specific local section of the sheaf $\mathbb{C}_{\lambda}$ on $T_{\mathbb{R}}^{\prime}$ has been chosen near $t$, and let us denote this section by the symbol $e^{\lambda-\rho}$, for simplicity. In this situation

$$
\begin{aligned}
e^{-(\lambda-\rho)} \phi_{t}: \mathcal{H}_{N^{\prime}(t, x)}^{i}(\mathbb{D} \mathcal{F}(x))_{x} \rightarrow \mathcal{H}_{N^{\prime}(t, x)}^{i}(\mathbb{D} \mathcal{F}(x))_{x}, \\
e^{-(\lambda-\rho)} \phi_{t}: \mathcal{H}_{\{x\}}^{i}\left(\left.(\mathbb{D} \mathcal{F}(x))\right|_{N^{\prime \prime}(t, x)}\right) \rightarrow \mathcal{H}_{\{x\}}^{i}\left(\left.(\mathbb{D} \mathcal{F}(x))\right|_{N^{\prime \prime}(t, x)}\right)
\end{aligned}
$$

are well defined, and

$$
\begin{gathered}
c_{t, x}=d_{t, x} e^{\lambda-\rho}, \text { with } \\
d_{t, x}=\sum(-1)^{i} \operatorname{tr}\left(e^{\rho-\lambda} \phi_{t}: \mathcal{H}_{N^{\prime}(t, x)}^{i}(\mathbb{D} \mathcal{F}(x))_{x} \rightarrow \mathcal{H}_{N^{\prime}(t, x)}^{i}(\mathbb{D} \mathcal{F}(x))_{x}\right)= \\
\sum(-1)^{i} \operatorname{tr}\left(e^{\rho-\lambda} \phi_{t}: \mathcal{H}_{\{x\}}^{i}\left(\left.(\mathbb{D} \mathcal{F}(x))\right|_{N^{\prime \prime}(t, x)}\right) \rightarrow \mathcal{H}_{\{x\}}^{i}\left(\left.(\mathbb{D} \mathcal{F}(x))\right|_{N^{\prime \prime}(t, x)}\right)\right) ;
\end{gathered}
$$

here $d_{t, x}$ is now a scalar. In particular, this applies for any regular $t \in T_{\mathbb{R}}$ near the identity: we choose the branch of $e^{\lambda-\rho}$ with value 1 at the identity. For such $t$, $e^{\rho-\lambda} \phi_{t}$ is homotopic to the identity map, so

$$
d_{t, x}=\chi\left(\mathcal{H}_{N^{\prime}(t, x)}^{*}(\mathbb{D} \mathcal{F}(x))_{x}\right)=\chi\left(\mathcal{H}_{\{x\}}^{*}\left(\left.\mathbb{D} \mathcal{F}(x)\right|_{N^{\prime \prime}(t, x)}\right)\right)
$$

becomes an Euler characteristic. The resulting formulas can be continued to the connected component of $t$ in $T_{\mathbb{R}}^{\prime}$. Every connected component $C$ of $T_{\mathbb{R}}^{\prime} \cap T_{\mathbb{R}}^{0}$ contains the identity in its closure, hence $c_{t, x}$ either vanishes identically on $C$, or equals an integral multiple of a well defined branch of $e^{\lambda-\rho}$. One can argue similarly for connected components of $T_{\mathbb{R}}^{\prime}$ outside $T_{\mathbb{R}}^{0}$, where $c_{t, x}$ can be expressed as a cyclotomic integer times a well defined branch of $e^{\lambda-\rho}$, unless $c_{t, x}$ vanishes identically.

The analogues on the Lie algebra of Theorems 5.12 and 5.24 are formally simpler, because the local system $\mathbb{C}_{\lambda}$ on $\tilde{\mathfrak{g}}$ has a canonical global generating section $e^{\lambda-\rho}$. We fix a particular point $(\zeta, x) \in \tilde{\mathfrak{g}}_{\mathbb{R}}{ }^{\prime}$, write $T$ for the centralizer of $\zeta$ in $G$, and define

$$
\begin{gathered}
N^{+}(\zeta, x)=N^{+}(\exp (\zeta), x), \\
N^{\prime}(\zeta, x)=N^{\prime}(\exp (\zeta), x), \quad N^{\prime \prime}(\zeta, x)=N^{\prime \prime}(\exp (\zeta), x) .
\end{gathered}
$$


Let $E$ be the connected component of $\mathfrak{t}_{\mathbb{R}}^{\prime}$ containing $\zeta$.

5.27 Theorem. For $\mathcal{F} \in \mathrm{D}_{G_{\mathbb{R}}}(X)_{-\lambda}$, the constant $d_{E, x}$ appearing in the local expression (4.4) of the virtual character $\theta=\theta(\mathcal{F})$ is given by the formula

$$
d_{E, x}=\chi\left(\mathcal{H}_{N^{\prime}(\zeta, x)}^{*}(\mathbb{D} \mathcal{F}(x))_{x}\right)=\chi\left(\mathcal{H}_{\{x\}}^{*}\left(\left.\mathbb{D} \mathcal{F}(x)\right|_{N^{\prime \prime}(t, x)}\right)\right) .
$$

The character cycle $c \in \mathrm{H}_{d}^{\text {inf }}\left(\tilde{\mathfrak{g}}_{\mathbb{R}}, \mathbb{C}_{-\lambda}\right)$ of the virtual character $\theta(\mathcal{F})$ is the product of the generating section $e^{\lambda-\rho}$ with an absolute cycle $c_{\rho} \in \mathrm{H}_{d}^{\text {inf }}\left(\tilde{\mathfrak{g}}_{\mathbb{R}}, \mathbb{C}\right)$, just as in the simplified version of Theorem 5.12 for integral $\lambda$ - see the discussion below the statement of that theorem. We shall omit a formal statement of the Lie algebra version of Theorem 5.12, since it is a direct analogue of the simplified statement with $\lambda$ integral - of 5.12 .

We remarked earlier that the hypotheses of Theorem 5.24 are unnecessarily restrictive. First of all, the condition on $\Psi^{\prime}$ and $\Psi^{\prime \prime}$ embodied by (5.15a) needs to be satisfied only by roots $\alpha \in \Phi^{+}$such that $e^{\alpha_{x}}$ is real valued and positive near $g$. To prove this more general version would be considerably more involved, since it does not directly follow from the fixed point formalism of either Goresky-MacPherson [GM] or Kashiwara [KSa]. Secondly, condition (5.15b) serves the purpose of making $\mathfrak{n}^{\prime}(g, x)$ and $\mathfrak{n}^{\prime \prime}(g, x)$ subalgebras, which is natural from a group theoretic point of view. This condition is used crucially in $\S 8$, where we show that Theorem 5.24 is compatible with parabolic induction. It is not used in $\S 6$, however, when we show that Theorems 5.12 and 5.24 are equivalent.

\section{Formal aspects of the Proofs}

In broad outline, we shall prove our main theorems - the character formulas 3.8, $5.12,5.24,5.27$ - by verifying them for certain "standard sheaves". In this section, we describe the standard sheaves, and we argue that they generate the K-groups of the equivariant derived categories $\mathrm{D}_{G_{\mathbb{R}}}(X)_{-\lambda}$. In subsequent sections we break up the verification for standard sheaves into several steps, which we then carry out.

To begin with, the main theorems descend to the level of the K-group: if three sheaves $\mathcal{F}_{i} \in \mathrm{D}_{G_{\mathbb{R}}}(X)_{-\lambda}$ fit into a distinguished triangle

$$
\mathcal{F}_{1} \rightarrow \mathcal{F}_{2} \rightarrow \mathcal{F}_{3} \rightarrow \mathcal{F}_{1}[1]
$$

and if any one of the main theorems holds for two of the $\mathcal{F}_{i}$, then it holds for the third. In the case of Theorem 3.8, this follows from (3.5) and the additivity of the integrals in 3.8. Theorems 5.24 and 5.27 involve alternating sums of traces, and these, too, behave additively ${ }^{8}$ in distinguished triangles. The additivity of 5.12 is less obvious; we establish it by appealing to the following proposition.

6.2 Proposition. Either of the two formulas for the $c_{t, x}$ in Theorem 5.24, for any particular sheaf $\mathcal{F} \in \mathrm{D}_{G_{\mathbb{R}}}(X)_{-\lambda}$, for all $x \in X$ and all regular $t$ fixing $x$, is equivalent to the statement of Theorem 5.12 for the sheaf $\mathcal{F}$.

We postpone the proof to the end of this section. Let us summarize our conclusions so far.

6.3 Remark. It suffices to establish the main theorems for a collection of sheaves which generate the K-group of $\mathrm{D}_{G_{\mathbb{R}}}(X)_{-\lambda}$.

\footnotetext{
${ }^{8}$ In the case of 5.24 , this depends on the functoriality of the morphism $\phi$, which insures that the induced maps on cohomology determine a morphism of the long exact cohomology sequences.
} 
Standard sheaves are associated to $G_{\mathbb{R}}$-orbits in the flag variety. Let $S \subset X$ be an orbit and $\hat{S} \subset \hat{X}$ its inverse image in the enhanced flag variety. We fix $\lambda \in \mathfrak{h}^{*}$ and consider an irreducible, $G_{\mathbb{R}}$-equivariant $(-\lambda-\rho)$-monodromic local system $\mathcal{L}$ on $S$-in other words, an irreducible, $G_{\mathbb{R}} \times \mathfrak{h}$-equivariant local system on $\hat{S}$ whose monodromy along the fibers of $\hat{S} \rightarrow S$ agrees with the monodromy of the multiple valued function $e^{-\lambda-\rho}$ on $H$. The direct image $R j_{*} \mathcal{L}$ of $\mathcal{L}$ under the inclusion $j$ of $S$ into $X$ is an object of the twisted equivariant derived category $\mathrm{D}_{G_{\mathbb{R}}}(X)_{-\lambda}$. By definition, the sheaves of this type are the standard sheaves in this category.

6.4 Lemma. The standard sheaves in $\mathrm{D}_{G_{\mathbb{R}}}(X)_{-\lambda}$ generate $K\left(\mathrm{D}_{G_{\mathbb{R}}}(X)_{-\lambda}\right)$. More precisely, the standard sheaves generate $\mathrm{D}_{G_{\mathbb{R}}}(X)_{-\lambda}$ as a triangulated category.

Proof. The support $\operatorname{Supp}(\mathcal{F})$ of any given $\mathcal{F} \in \mathrm{D}_{G_{\mathbb{R}}}(X)_{-\lambda}$ is necessarily $G_{\mathbb{R}^{-}}$ invariant. Let $S=\bigcup S_{j}$ be the union of the orbits $S_{j}$ of maximal dimension in $\operatorname{Supp}(\mathcal{F}), \quad j: S \hookrightarrow X$ the inclusion. The natural morphism $\mathcal{F} \rightarrow R j_{*} j^{*} \mathcal{F}$ induces an isomorphism over $S$, hence the third term in the distinguished triangle

$$
\mathcal{F} \rightarrow R j_{*} j^{*} \mathcal{F} \rightarrow \mathcal{G} \rightarrow \mathcal{F}[1]
$$

has support of strictly lower dimension than $\operatorname{Supp}(\mathcal{F})$, or vanishes identically. Arguing by induction, we may now assume that $\mathcal{F}=R j_{*} j^{*} \mathcal{F}$. The cohomology sheaves of $j^{*} \mathcal{F} \in \mathrm{D}_{G_{\mathbb{R}}}(S)_{-\lambda}$ are $G_{\mathbb{R}}$-equivariant, $(-\lambda-\rho)$-monodromic local systems on $S$. As such, they must be extensions of irreducible local systems on the various $S_{j}$. The lemma follows.

In spite of the geometric terminology, irreducible $G_{\mathbb{R}}$-equivariant, $(-\lambda-\rho)$-monodromic local systems are essentially algebraic objects. We recall how to construct them in terms of Lie theoretic data. This involves, first of all, the enumeration of the $G_{\mathbb{R}}$-orbits in $X$ as in [Ma], [W]. Let $T_{\mathbb{R}}$ be a Cartan subgroup, and $\tau_{x_{0}}: \mathfrak{t} \rightarrow \mathfrak{h}$ a concrete isomorphism between the complexified Lie algebra $\mathfrak{t}$ of $T_{\mathbb{R}}$ and the universal Cartan $\mathfrak{h}$, corresponding to a fixed point $x_{0} \in X$ of $T_{\mathbb{R}}$. Note that the $G_{\mathbb{R}}$-orbit

$$
S=S\left(T_{\mathbb{R}}, \tau_{x_{0}}\right)=G_{\mathbb{R}} \cdot x_{0}
$$

remains unchanged when $T_{\mathbb{R}}$ is replaced by a $G_{\mathbb{R}}$-conjugate or $x_{0}$ by a $N_{G_{\mathbb{R}}}\left(T_{\mathbb{R}}\right)$ translate. The correspondence $\left(T_{\mathbb{R}}, \tau_{x_{0}}\right) \mapsto S$ sets up a bijection between the set of $G_{\mathbb{R}}$-orbits and the set of pairs $\left(T_{\mathbb{R}}, \tau_{x_{0}}\right)$, modulo the conjugacies just mentioned.

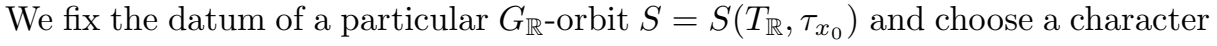
$\chi: T_{\mathbb{R}} \rightarrow \mathbb{C}^{*}$ such that $d \chi=\tau_{x_{0}}^{*}(\lambda-\rho)$, as complex linear functions on $\mathfrak{t}$. A parenthetical comment: if $G_{\mathbb{R}}$ is nonlinear, contrary to our standing assumption, $T_{\mathbb{R}}$ may fail to be abelian; in that case, we need to allow irreducible representations $\chi$ of dimension greater than one, with differential equal to a direct sum of copies of $\tau_{x_{0}}^{*}(\lambda-\rho)$. The isotropy subgroup of $G_{\mathbb{R}}$ at $x_{0} \in X$ splits into a direct product

$$
\left(G_{\mathbb{R}}\right)_{x_{0}}=T_{\mathbb{R}} \cdot\left(N_{x_{0}} \cap G_{\mathbb{R}}\right),
$$

with $N_{x_{0}}=\exp \left[\mathfrak{b}_{x_{0}}, \mathfrak{b}_{x_{0}}\right]$. The group $N_{x_{0}} \cap G_{\mathbb{R}}$ is connected, so $\chi$ lifts canonically to a character $\tilde{\chi}:\left(G_{\mathbb{R}}\right)_{x_{0}} \rightarrow \mathbb{C}^{*}$, with $\tilde{\chi}=1$ on $N_{x_{0}} \cap G_{\mathbb{R}}$. When we identify the enhanced flag variety $\hat{X}$ with $G / N_{x_{0}}$ as usual, the identity coset corresponds to a point $\hat{x_{0}} \in \hat{X}$ lying over $x_{0}$. In particular, $\hat{x_{0}}$ lies in $\hat{S}$, the inverse image of $S$ in $\hat{X}$. The group $G_{\mathbb{R}} \times \mathfrak{h}$ acts transitively on $\hat{S}$, with isotropy group

$$
\begin{aligned}
\left(G_{\mathbb{R}} \times \mathfrak{h}\right)_{\hat{x_{0}}} & =\left\{(g, \zeta) \in\left(G_{\mathbb{R}}\right)_{x_{0}} \times \mathfrak{h} \mid g=\exp \left(\tau_{x_{0}}^{-1} \zeta\right)\right\} \\
& =\left\{(t, n, \zeta) \in T_{\mathbb{R}} \times\left(N_{x_{0}} \cap G_{\mathbb{R}}\right) \times \mathfrak{h} \mid t=\exp \left(\tau_{x_{0}}^{-1} \zeta\right)\right\}
\end{aligned}
$$


at $\hat{x_{0}}$. A triple $(t, n, \zeta)$ lies in the identity component precisely when $\tau_{x_{0}}^{-1} \zeta \in \mathfrak{t}_{\mathbb{R}}$, hence

$$
\begin{aligned}
& \text { component group of }\left(G_{\mathbb{R}} \times \mathfrak{h}\right)_{\hat{x_{0}}} \cong \\
& \left\{(t, \zeta) \in T_{\mathbb{R}} \times \mathfrak{h} \mid t=\exp \left(\tau_{x_{0}}^{-1} \zeta\right)\right\} /\left\{(t, \zeta) \mid t=\exp \left(\tau_{x_{0}}^{-1} \zeta\right), \tau_{x_{0}}^{-1} \zeta \in \mathfrak{t}_{\mathbb{R}}\right\} .
\end{aligned}
$$

Note that the character

$$
\psi:\left(G_{\mathbb{R}} \times \mathfrak{h}\right)_{\hat{x_{0}}} \rightarrow \mathbb{C}^{*}, \quad \psi(g, \zeta)=\tilde{\chi}(g) e^{-(\lambda-\rho)(\zeta)}
$$

is identically equal to 1 on the identity component of $\left(G_{\mathbb{R}} \times \mathfrak{h}\right)_{\hat{x_{0}}}$, and thus induces a character of the component group. As such, it defines a $G_{\mathbb{R}} \times \mathfrak{h}$-equivariant local system $\mathcal{L}_{\chi}$ on $\hat{S}$, of rank 1 , which is therefore irreducible.

6.9 Lemma. The local system $\mathcal{L}_{\chi}$ is $(-\lambda-\rho)$-monodromic. Every irreducible $G_{\mathbb{R}}$ equivariant, $(-\lambda-\rho)$-monodromic local system on $S$ is of this form, with uniquely determined $\chi$.

Proof. Because of the $G_{\mathbb{R}}$-equivariance, it suffices to check the monodromicity condition on the fiber over $x_{0}$. On that fiber it has the monodromy of the function $e^{-(\lambda-\rho)}$, hence the monodromy of $e^{-(\lambda+\rho)}$ since $2 \rho$ is integral. Conversely, the datum of an irreducible $G_{\mathbb{R}^{-}}$equivariant, $(-\lambda-\rho)$-monodromic local system on $S$ is equivalent to that of an irreducible representation $\psi$ of the component group (6.7), subject to the condition

$$
\psi(e, \zeta)=e^{-(\lambda-\rho)(\zeta)} \text { for } \zeta \in \mathfrak{h}_{\mathbb{Z}}
$$

which reflects the monodromicity condition (recall: $\mathfrak{h}_{\mathbb{Z}}$ is the kernel of the exponential map exp $: \mathfrak{h} \rightarrow H$ ). Note that $\psi$ must be one-dimensional since the component group is abelian. We reconstruct the datum of the character $\chi: T_{\mathbb{R}} \rightarrow \mathbb{C}^{*}$ by defining $\chi(t)=\psi(t, \zeta) e^{(\lambda-\rho)(\zeta)}$ for any $\zeta \in \mathfrak{h}$ such that $t=\exp \left(\tau_{x_{0}}^{-1}(\zeta)\right)$ - the condition (6.10) ensures that the particular choice of $\zeta$ does not matter. When $t$ happens to lie in the identity component of $T_{\mathbb{R}}$, we can choose $\zeta$ to lie in $\tau_{x_{0}}\left(\mathfrak{t}_{\mathbb{R}}\right)$, in which case $\psi(t, \zeta)=1$. This shows that $d \chi=\tau_{x_{0}}^{*}(\lambda-\rho)$, as required.

In effect, the lemma gives us an explicit description of the standard sheaves in $\mathrm{D}_{G_{\mathbb{R}}}(S)_{-\lambda}$. To complete the verification of 6.3 , we still need to prove Proposition 6.2. This involves two major steps: the reduction from the general, twisted situation to the untwisted case, and secondly, the computation of the local fixed point contribution in the untwisted case. This second ingredient already appears in Kashiwara's announcement [Ka4].

Proof of Proposition 6.2. Let us assume that the statement of Theorem 5.24 is satisfied for a particular $\mathcal{F} \in \mathrm{D}_{G_{\mathbb{R}}}(S)_{-\lambda}$ and all possible choices of a Cartan subgroup $T_{\mathbb{R}} \subset G_{\mathbb{R}}$, of a regular element $t \in T_{\mathbb{R}}^{\prime}$, and of fixed point $x$ of $T$. We must show that the character identity in Theorem 5.12 holds, and conversely, that the identity in Theorem 5.12 implies the statement of Theorem 5.24 for all data $T_{\mathbb{R}}, t \in T_{\mathbb{R}}^{\prime}$, and $x \in X^{T}$. The virtual character $\Theta=\Theta(\mathcal{F})$ is completely determined by its values on the regular set, where it is real analytic. The value of the integral on the right of the identity is also completely determined by the contribution lying over the regular set: the inverse image of the singular set in $\tilde{G}_{\mathbb{R}}$ has codimension at least one, and can therefore be neglected in the integral. In particular, instead of considering arbitrary test functions in the character identity, it suffices to consider the case when the test function $\phi$ is a delta function supported at an arbitrary 
regular point - say $\phi=\delta_{t}$ with $t \in T_{\mathbb{R}}^{\prime}$ as before. In this situation, the left hand side of the identity reduces to

$$
\int_{G_{\mathbb{R}}} \Theta \delta_{t} d g=\Theta(t)=\sum_{x \in X^{T}} \frac{c_{t, x}}{\prod_{\alpha \in \Phi^{+}}\left(1-e^{-\alpha_{x}}\right)(t)}
$$

with the coefficients $c_{t, x}$ as in (4.11). The right hand side also splits into a sum of terms indexed by the fixed points of $T$ :

$$
\int_{c(\mathcal{F})}\left(q^{*} \delta_{t}\right) \tilde{\omega}=\sum_{x \in X^{T}} \frac{\text { value of the cycle } c(\mathcal{F}) \text { at }(t, x)}{\prod_{\alpha \in \Phi^{+}}\left(1-e^{-\alpha_{x}}\right)(t)} .
$$

Here, as in $\S 4$, we regard the cycle $c(\mathcal{F})$ as a section of the local system $\mathbb{C}_{\lambda}$ via Poincare duality. The value of the cycle is actually a number, since the local system $\mathbb{C}_{\lambda}$ is a subsheaf of the sheaf of functions. At this point, the proposition comes down to the assertion that the two Lefschetz numbers

$$
\begin{aligned}
& \sum(-1)^{i} \operatorname{tr}\left(\phi_{t}: \mathcal{H}_{N^{\prime}(t, x)}^{i}(\mathbb{D} \mathcal{F}(x))_{x} \rightarrow \mathcal{H}_{N^{\prime}(t, x)}^{i}(\mathbb{D} \mathcal{F}(x))_{x} \otimes \mathbb{C}_{\lambda}\right), \\
& \sum(-1)^{i} \operatorname{tr}\left(\phi_{t}: \mathcal{H}_{\{x\}}^{i}\left(\left.(\mathbb{D} \mathcal{F}(x))\right|_{N^{\prime \prime}(t, x)}\right) \rightarrow \mathcal{H}_{\{x\}}^{i}\left(\left.(\mathbb{D} \mathcal{F}(x))\right|_{N^{\prime \prime}(t, x)}\right) \otimes \mathbb{C}_{\lambda}\right)
\end{aligned}
$$

coincide and equal the value of the cycle $c(\mathcal{F})$ at $(t, x)$. The verification of this assertion is our remaining task in this section.

We use the notation established in (5.13)-(5.18). In particular, $N^{+}(t, x) \times H$ is the inverse image of $N^{+}(t, x)$ in $\hat{X}$. The inclusion $\{t\} \hookrightarrow G_{\mathbb{R}}$, corresponding to a particular $t \in T_{\mathbb{R}}^{\prime}$, determines a Cartesian square

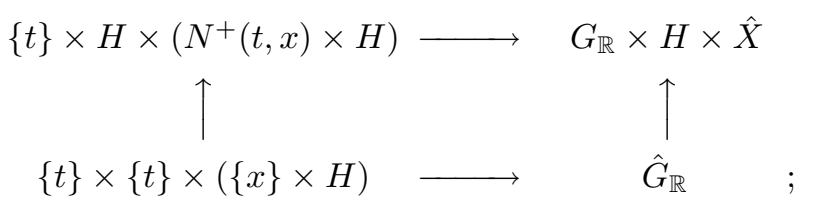

here we are using the formula (5.18) for the $T \times H$-action on $N^{+}(t, x) \times H$. Combining this with (5.9) gives the commutative diagram

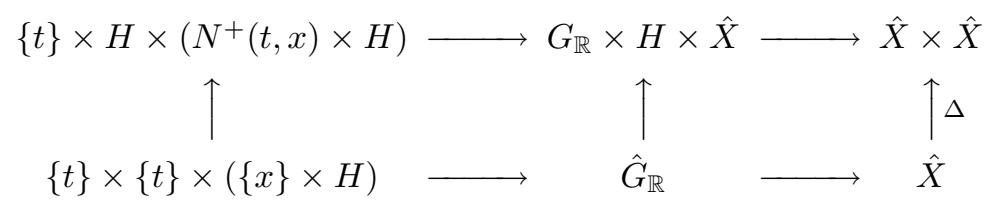

involving three Cartesian squares: the right square, the left square, and the square formed by the four terms on the perimeter - the "outer square" for short. The outer square is formally analogous to the right square. We can therefore apply the fixed point formalism (5.10). The result is a map ${ }^{9}$

$$
\operatorname{Hom}(\mathcal{G}, \mathcal{G}) \longrightarrow \mathrm{H}_{\hat{t}}^{0}\left(A_{t}, p^{*} \mathbb{D}_{\hat{X}} \otimes \mathbb{C}_{\lambda}\right) \cong \mathrm{H}_{0}^{i n f}\left(\{(t, x)\}, \mathbb{C}_{-\lambda}\right)
$$

with the shorthand notation

$$
A_{t}=\{t\} \times H \times\left(N^{+}(t, x) \times H\right), \quad \hat{t}=\{t\} \times\{t\} \times(\{x\} \times H) .
$$

To keep the notation simple, we are using the symbols $a, p$ also for the action and projection morphisms in the outer square.

\footnotetext{
${ }^{9}$ Recall our comment in $\S 5$, to the effect that we no longer make a notational distinction between $\mathcal{G} \in \mathrm{D}_{G_{\mathbb{R}}}(X)_{\lambda}$ and its image in $\mathrm{D}^{b}(\hat{X})$, as we had earlier in $\S 5$.
} 
We claim: when we combine (6.15) with its precursor in (5.10), the resulting diagram,

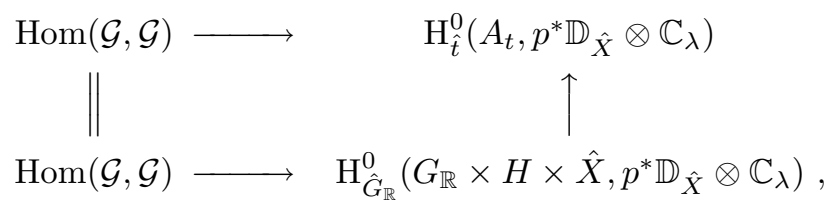

commutes. The reason is simply the functoriality of the fixed point formalism, which follows from the functorial behavior of local cohomology. We continue the bottom row in (6.16) as in (5.10), then restrict to the open subset ${\tilde{G_{\mathbb{R}}}}^{\prime}$ and apply Poincaré duality on this open subset, which consists of smooth points:

$$
\begin{aligned}
& \mathrm{H}_{\hat{G}_{\mathbb{R}}^{0}}^{0}\left(G_{\mathbb{R}} \times H \times \hat{X}, p^{*} \mathbb{D}_{\hat{X}} \otimes \mathbb{C}_{\lambda}\right) \stackrel{\sim}{\rightarrow} \mathrm{H}_{d+r}^{i n f}\left(\hat{G}_{\mathbb{R}}, \mathbb{C}_{-\lambda}\right) \\
\stackrel{\sim}{\rightarrow} & \mathrm{H}_{d}^{i n f}\left(\tilde{G}_{\mathbb{R}}, \mathbb{C}_{-\lambda}\right) \rightarrow \mathrm{H}_{d}^{i n f}\left(\tilde{G}_{\mathbb{R}}^{\prime}, \mathbb{C}_{-\lambda}\right) \stackrel{\sim}{\rightarrow} \mathrm{H}^{0}\left(\tilde{G}_{\mathbb{R}}^{\prime}, \mathbb{C}_{\lambda}\right) .
\end{aligned}
$$

We analogously continue the top row,

$$
\mathrm{H}_{\hat{t}}^{0}\left(A_{t}, p^{*} \mathbb{D}_{\hat{X}} \otimes \mathbb{C}_{\lambda}\right) \stackrel{\sim}{\rightarrow} \mathrm{H}_{0}^{\text {inf }}\left(\{(t, x)\}, \mathbb{C}_{-\lambda}\right) \stackrel{\sim}{\rightarrow} \mathrm{H}^{0}\left(\{(t, x)\}, \mathbb{C}_{\lambda}\right) .
$$

Putting $(6.17 \mathrm{a}, \mathrm{b})$ together, we obtain the diagram

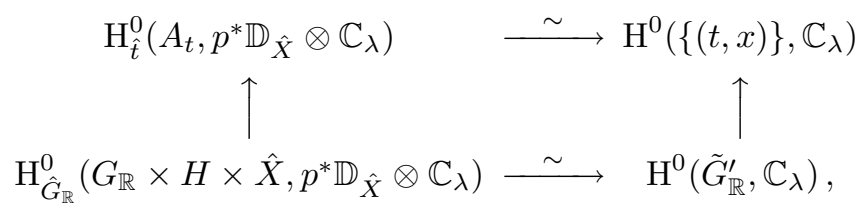

in which the second vertical arrow is evaluation of sections at $(t, x)$. This diagram commutes because of the functoriality of the various ingredients. Letting $\mathbb{D} \mathcal{F}$ play the role of $\mathcal{G}$, we conclude that the value of the cycle $c(\mathcal{F})$ at $(t, x)$ is given by the image of the identity morphism $1: \mathbb{D} \mathcal{F} \rightarrow \mathbb{D} \mathcal{F}$ under the chain of morphisms

$$
\begin{gathered}
\operatorname{Hom}(\mathbb{D} \mathcal{F}, \mathbb{D} \mathcal{F}) \rightarrow \mathrm{H}_{\hat{t}}^{0}\left(A_{t}, p^{*} \mathbb{D}_{\hat{X}} \otimes \mathbb{C}_{\lambda}\right) \\
\stackrel{\sim}{\rightarrow} \mathrm{H}^{0}\left(\{(t, x)\}, \mathbb{C}_{\lambda}\right) \stackrel{\sim}{\rightarrow} \mathbb{C} .
\end{gathered}
$$

It remains to be shown that this image of the identity coincides with the Lefschetz number (6.12). The final isomorphism in (6.19), "evaluation at $t$ ", makes the image of the identity morphism a specific number; here we are using the definition of $\mathbb{C}_{\lambda}$ as a subsheaf of the sheaf of functions.

The morphisms in (6.19) were obtained by applying the fixed point formalism (5.10) to the outer square in (6.14). Since the image of $\{t\} \times H \times\left(N^{+}(t, x) \times H\right)$ lies in the open subset $\left(N^{+}(t, x) \times H\right) \times\left(N^{+}(t, x) \times H\right)$ of $\hat{X} \times \hat{X}$, we may as well replace $\hat{X}$ by $N^{+}(t, x) \times H$ and $\mathcal{F}$ by its restriction to $N^{+}(t, x) \times H$. Then, when we apply the fixed point formalism in the Cartesian square

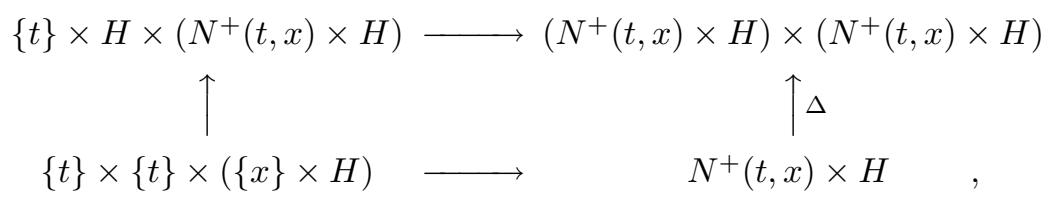

we obtain the same maps as in (6.19). Next we restrict the twisted sheaf $\mathcal{G}=\mathbb{D} \mathcal{F}$ from $N^{+}(t, x) \times H$ to $N^{+}(t, x) \times\{e\} \cong N^{+}(t, x)$, as in (5.19b), resulting in the sheaf $\mathcal{G}(x) \in \mathrm{D}_{T_{\mathbb{R}}}\left(N^{+}(t, x)\right)$. Correspondingly, we take a slice of the diagram (6.20) by replacing $N^{+}(t, x) \times H$ with $N^{+}(t, x) \times\{e\} \cong N^{+}(t, x)$ and $\{t\} \times H$ by $\{t\} \times\{t\}$. 
In this slice, the action and projection maps induce $a, p: N^{+}(t, x) \rightarrow N^{+}(t, x)$, with $a=$ left translation by $t, p=$ identity. We use these maps to construct the Cartesian square

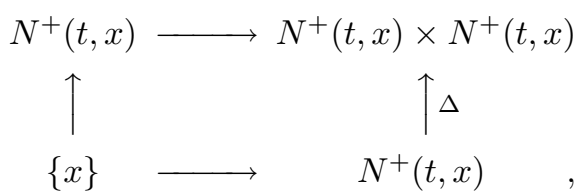

which maps into the Cartesian square (6.20) by inclusion. Recall the construction of the isomorphism (5.21) by means of the commutative diagram (5.20). We use the notation $\phi: t^{*} \mathcal{G}(x) \rightarrow \mathcal{G}(x)$ for the induced morphism when we restrict from $T_{\mathbb{R}}$ to $\{t\}$. The sheaf $\mathbb{C}_{\lambda}$ disappears at this point since $\left.\mathbb{C}_{\lambda}\right|_{\{t\}} \cong \mathbb{C}$ by evaluation at $t$. When we apply the fixed point formalism to the square (6.21) instead of (5.9), $\phi$ induces a morphism

$$
\operatorname{Hom}(\mathbb{D} \mathcal{F}(x), \mathbb{D} \mathcal{F}(x)) \longrightarrow \mathrm{H}_{\{x\}}^{0}\left(N^{+}(t, x), \mathbb{D}_{N^{+}(t, x)}\right) \stackrel{\sim}{\longrightarrow},
$$

analogous to (6.19).

We claim: The image of $1 \in \operatorname{Hom}(\mathbb{D} \mathcal{F}(x), \mathbb{D} \mathcal{F}(x))$ under the composite morphism in (6.22) coincides with the image of the identity under the chain of homomorphisms (6.19). The crux of the matter is the functoriality of the fixed point formalism with respect to noncharacteristic maps - in our case, $N^{+}(t, x) \hookrightarrow N^{+}(t, x) \times H-$ a general fact which can be verified by tracing through diagrams, though not entirely without effort. The inclusion $N^{+}(t, x) \hookrightarrow N^{+}(t, x) \times H$ relates the two chains of morphisms (6.19) and (6.22), so our claim follows from the functoriality properties of the fixed point formalism.

To complete the proof, we still must identify the Lefschetz numbers (6.12) with the image of the identity under the homomorphism (6.22). The equality of the three quantities follows from an appropriate generalization of the Lefschetz fixed point theorem. Generalizations that apply in our situation have been given by Kashiwara [Ka4], [KSa] and Goresky-MacPherson [GM]. Kashiwara's fixed point formalism expresses the global Lefschetz number as a sum over local contributions corresponding to the components of the fixed point sets; in the case of isolated fixed points - which is the case of interest to us - he gives an explicit description of the local contributions. Specifically, the local contribution corresponding to an isolated fixed point $x$ is the image of the identity under the homomorphism (6.22); further, Kashiwara identifies this local contribution with Lefschetz numbers similar to (6.12), but specialized to the tangent space at $x$. Goresky-MacPherson also express the global Lefschetz number as a sum of local contributions. In the case of an isolated fixed point $x$, their local contribution coincides precisely with either of the two local Lefschetz numbers (6.12) [GM, formulas for $A_{4}$ and $A_{5}$ ]. They also establish the uniqueness of the local contributions, provided they are expressed in terms of local data near the fixed point [GM, §5.1]. In particular, their local Lefschetz numbers coincide with Kashiwara's. This gives us the conclusion we need.

\section{The CASE OF THE DisCRETE SERIES}

In this section we establish our character formulas for standard sheaves associated to certain $G_{\mathbb{R}}$-orbits $S$ and certain twisting parameters $\lambda$ : orbits $S$ attached to a compact Cartan subgroup $T_{\mathbb{R}}$ - these are necessarily open - and any regular 
anti-dominant $\lambda$. Such geometric data correspond to discrete series representations. This particular case of Theorems 5.12 and 5.24 was already established by Kashiwara in his announcement [Ka4].

Then let $T_{\mathbb{R}} \subset G_{\mathbb{R}}$ be a compact Cartan subgroup, and $S=S\left(T_{\mathbb{R}}, \tau_{x_{0}}\right)$ a $G_{\mathbb{R}}$-orbit corresponding to the datum of $T_{\mathbb{R}}$ and of a fixed point $x_{0}$ of $T_{\mathbb{R}}$, as in (6.5). Our hypotheses on $G_{\mathbb{R}}$ imply that the compact Cartan subgroup $T_{\mathbb{R}}$ is connected, hence a torus. As was argued in $\S 6$, standard sheaves associated to $S$ and any particular twisting parameter $\lambda$ correspond bijectively to $G_{\mathbb{R}}$-equivariant, irreducible, $(-\lambda-$ $\rho$ )-monodromic local systems on $S$, and those, in turn, correspond bijectively to characters $\chi: T_{\mathbb{R}} \rightarrow \mathbb{C}^{*}$ whose differentials coincide with $\lambda-\rho$. Because of the

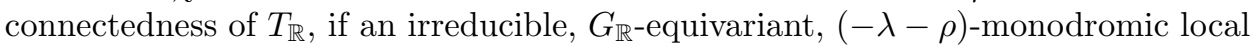
system on $S$ exists, it is unique; moreover, such a local system exists precisely when $\lambda-\rho$ is an $H$-integral weight. ${ }^{10}$ Thus, without loss of generality, we assume

$$
\lambda \in \Lambda+\rho \quad(\Lambda=\text { weight lattice of } H) .
$$

Let $j: S \hookrightarrow X$ denote the inclusion. We can appeal to (2.12)-(2.13), and conclude that $R j_{*} \mathbb{C}_{S} \in \mathrm{D}_{G_{\mathbb{R}}}(X)=\mathrm{D}_{G_{\mathbb{R}}}(X)_{-\lambda}$ is the only standard sheaf in $\mathrm{D}_{G_{\mathbb{R}}}(X)_{-\lambda}$ associated to the orbit $S$.

Recall (2.15) and the discussion below it: because of the integrality of $\lambda+\rho$, the twisted sheaf $\mathcal{O}(\lambda)$ becomes an actual sheaf on $X$ with a $G$-action, and as such coincides with the sheaf of holomorphic sections of the $G$-equivariant line bundle $\mathbf{L}_{\lambda-\rho}$ on $X$. Hence, with $\mathcal{F}=R j_{*} \mathbb{C}_{S}$, the construction (2.16)-(2.17) produces the virtual character

$$
\begin{aligned}
\Theta\left(R j_{*} \mathbb{C}_{S}\right) & =\sum_{p}(-1)^{p} \Theta\left(\operatorname{Ext}^{p}\left(\mathbb{D} R j_{*} \mathbb{C}_{S}, \mathcal{O}_{X}(\lambda)\right)\right) \\
& =\sum_{p}(-1)^{p} \Theta\left(\operatorname{Ext}^{p}\left(R j_{!} \mathbb{C}_{S}\left[2 \operatorname{dim}_{\mathbb{C}} X\right], \mathcal{O}_{X}\left(\mathbf{L}_{\lambda-\rho}\right)\right)\right) \\
& =\sum_{p}(-1)^{p} \Theta\left(\operatorname{Ext}^{p}\left(\mathbb{C}_{S}, j^{\prime} \mathcal{O}_{X}\left(\mathbf{L}_{\lambda-\rho}\right)\right)\right) \\
& =\sum_{p}(-1)^{p} \Theta\left(\operatorname{Ext}^{p}\left(\mathbb{C}_{S}, j^{*} \mathcal{O}_{X}\left(\mathbf{L}_{\lambda-\rho}\right)\right)\right) \\
& =\sum_{p}(-1)^{p} \Theta\left(\mathrm{H}^{p}\left(S, \mathcal{O}_{X}\left(\mathbf{L}_{\lambda-\rho}\right)\right)\right) .
\end{aligned}
$$

Here we have used the fact that an even shift in degrees does not affect the Euler characteristic, the adjointness of $R j$ ! and $j^{!}$, and the equality of $j^{!}$and $j^{*}$ for open embeddings.

Since $T_{\mathbb{R}}$ is a compact Cartan subgroup of the linear group $G_{\mathbb{R}}, \tau_{x_{0}}^{*} \lambda$ is the full weight lattice of the torus $T_{\mathbb{R}}$, and $\tau_{x_{0}}^{*}(\Lambda+\rho)$ the weight lattice of $T_{\mathbb{R}}$ shifted by the half-sum of the positive roots. To each regular $\mu \in \tau_{x_{0}}^{*}(\Lambda+\rho)$, Harish-Chandra [HC6] associates a discrete series representation $\pi_{\mu}$, whose character he denotes by the symbol $\Theta_{\mu}$; every discrete series character is of this type for some regular $\mu \in \tau_{x_{0}}^{*}(\Lambda+\rho)$, and $\Theta_{\mu}=\Theta_{\nu}$ if and only if the parameters $\mu, \nu$ are conjugate under the normalizer of $T_{\mathbb{R}}$ in $G_{\mathbb{R}}$. The integrality condition (7.1) on the parameter $\lambda$ in (7.2) implies that $2 \frac{(\lambda, \alpha)}{(\alpha, \alpha)}$ is an integer for each $\alpha \in \Phi$. In addition to (7.1), we now impose the condition that $\lambda$ is regular anti-dominant, in the sense that

$$
2 \frac{(\lambda, \alpha)}{(\alpha, \alpha)} \in \mathbb{Z}_{<0} \quad \text { for all } \alpha \in \Phi^{+} .
$$

\footnotetext{
${ }^{10}$ In the linear case, compact Cartan subgroups of $G_{\mathbb{R}}$ have the same weight lattice as the universal Cartan $H$; if, contrary to our assumptions, $G_{\mathbb{R}}$ fails to be linear, the phrase " $H$-integral" should be replaced by " $G_{\mathbb{R}}$-integral".
} 
For every $\lambda \in \Lambda+\rho$ which satisfies this anti-dominance condition, we define

$$
\Theta(S, \lambda)=\Theta_{\tau_{x_{0}}^{*} \lambda}
$$

Two comments are in order. First, the open $G_{\mathbb{R}}$-orbit $S$ determines the pair $\left(T_{\mathbb{R}}, x_{0}\right)$

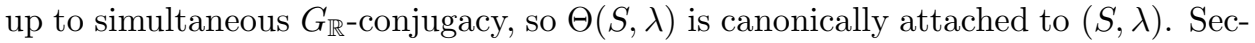
ondly, we insist on the anti-dominance condition (7.3) in the definition (7.4) because we want $\Theta(S, \lambda)$ to depend coherently on the parameter $\lambda$; see $\S 8$. This has the added advantage of making our parametrization of the discrete series, in terms of pairs $(S, \lambda)$, one-to-one.

The compact Cartan subgroup $T_{\mathbb{R}}$ lies in a maximal compact subgroup of $G_{\mathbb{R}}$; we may as well assume that it is the one we had already chosen: $T_{\mathbb{R}} \subset K_{\mathbb{R}}$. Then $K_{\mathbb{R}} / T_{\mathbb{R}}$ can be identified with the flag variety of $\mathfrak{k}$. In particular, the (real) dimensions of $K_{\mathbb{R}} / T_{\mathbb{R}}$ and $G_{\mathbb{R}} / K_{\mathbb{R}}$ are even. We set

$$
s=\frac{1}{2} \operatorname{dim} K_{\mathbb{R}} / T_{\mathbb{R}}, \quad q=\frac{1}{2} \operatorname{dim} G_{\mathbb{R}} / K_{\mathbb{R}} .
$$

Note that the virtual character $\Theta\left(R j_{*} \mathbb{C}_{S}\right)$ in (7.2) depends on the choice of $\lambda$, even though $\lambda$ does not appear in the notation: we have chosen to regard $R j_{*} \mathbb{C}_{S}$ as an object in $\mathrm{D}_{G_{\mathbb{R}}}(X)_{-\lambda}$.

7.6 Theorem. Under the hypotheses just stated, $\Theta\left(\mathrm{H}^{s}\left(S, \mathcal{O}_{X}\left(\mathbf{L}_{\lambda-\rho}\right)\right)\right)=\Theta(S, \lambda)$, and $\mathrm{H}^{p}\left(S, \mathcal{O}_{X}\left(\mathbf{L}_{\lambda-\rho}\right)\right)=0$ if $p \neq s$. In particular, $\Theta\left(R j_{*} \mathbb{C}_{S}\right)=(-1)^{s} \Theta(S, \lambda)$.

When $\lambda$ is not only anti-dominant but also "very regular", i.e., if $|(\lambda, \alpha)| \gg 0$ for all $\alpha \in \Phi$, this is the main result of [S1] in conjunction with the characterization of discrete series representations by lowest $K$-type [S2], [V]. The general case then follows from the Jantzen-Zuckerman translation principle, as in [S3], for example. Alternatively, the theorem is a consequence of the main result in [KSd].

In the remainder of this section, we shall verify the two character formulas in the present situation, i.e., with $\operatorname{rk} G_{\mathbb{R}}=\operatorname{rk} K_{\mathbb{R}}, S \subset X$ open, $\mathcal{F}=R j_{*} \mathbb{C}_{S}$, and $\lambda$ satisfying the anti-dominance condition (7.3). We first deal with the case of the integral formula (3.8).

The decomposition $\mathfrak{g}=\mathfrak{t} \oplus[\mathfrak{t}, \mathfrak{g}]$ permits us to view $\tau_{x_{0}}^{*} \lambda$ as an element of $\mathfrak{g}^{*}-$ an element of $i \mathfrak{g}_{\mathbb{R}}^{*}$, in fact, since differentials of characters of $T_{\mathbb{R}}$ are purely imaginary. Recall that $\Omega_{\lambda}$ denotes the $G$-orbit of $\tau_{x_{0}}^{*} \lambda$ in $\mathfrak{g}^{*}$. We define

$$
\Omega(S, \lambda)=G_{\mathbb{R}} \text {-orbit of } \tau_{x_{0}}^{*} \lambda \text { in } i \mathfrak{g}_{\mathbb{R}}^{*} .
$$

As in the case of $\Theta(S, \lambda)$, the notation is justified because $S$ determines $T_{\mathbb{R}}$ and $\tau_{x_{0}}$ up to simultaneous $G_{\mathbb{R}^{-}}$-conjugacy. Let us note that the union of the $\Omega(S, \lambda)$, corresponding to all the open $G_{\mathbb{R}}$-orbits in $X$, coincides with $\Omega_{\lambda} \cap i \mathfrak{g}_{\mathbb{R}}^{*}$. Recall that the complex coadjoint orbit $\Omega_{\lambda}$ comes equipped with a canonical algebraic symplectic form $\sigma_{\lambda}$. Since $\lambda$ is regular, the orbit $\Omega_{\lambda}$ has complex dimension $2 n$, where $n=\operatorname{dim}_{\mathbb{C}} X$, as before. Also recall our convention (3.6) - without the choice of $i=\sqrt{-1}-$ for the Fourier transform $\hat{\phi}$. The following result is due to Rossmann [R1].

7.8 Theorem. The two-form $-i \sigma_{\lambda}$ restricts to a real, nondegenerate form on the real submanifold $\Omega(S, \lambda) \subset \Omega_{\lambda}$. Orient $\Omega(S, \lambda)$ so as to make the top exterior power of $-i \sigma_{\lambda}$ positive. Let $\theta(S, \lambda)$ be the character on the Lie algebra corresponding to 
the discrete series character $\Theta(S, \lambda)$. Then, for $\phi \in C_{c}^{\infty}\left(\mathfrak{g}_{\mathbb{R}}\right)$,

$$
\int_{\mathfrak{g}_{\mathbb{R}}} \theta(S, \lambda) \phi d x=\frac{1}{(2 \pi i)^{n} n !} \int_{\Omega(S, \lambda)} \hat{\phi} \sigma_{\lambda}^{n}
$$

the integral on the right converges absolutely.

The definition of $\hat{\phi}$ involves the choice of Euclidean measure $d x$ on $\mathfrak{g}_{\mathbb{R}}$; we are using the same measure on the left of the above identity, of course.

We shall use the open embedding theorem of [SV4, theorem 4.2] to relate the inverse image of $\Omega(S, \lambda)$ under the twisted moment map $\mu_{\lambda}$ to the characteristic cycle of the sheaf $R j_{*} \mathbb{C}_{S}$. The crux of the matter is the following technical result. The statement involves a real valued function $f_{\lambda}$ on $S$ which we now define. The parameter $\lambda$ lies in $\Lambda+\rho$, and $\rho$ is integral, if not for $G$, then at least for some 2-fold covering of the complex group $G$. We conclude that the line bundle $\mathbf{L}_{\lambda}$ exists as an algebraic line bundle on $X$, equivariant with respect to $G$ or a 2-fold covering of $G$. By assumption, $G_{\mathbb{R}}$ acts on $S$ with compact isotropy groups. Replacing $G_{\mathbb{R}}$ by its inverse image in the 2-fold covering of $G$ if necessary, we find that $\left.\mathbf{L}_{\lambda}\right|_{S}$ admits a $G_{\mathbb{R}}$-invariant Hermitian metric $\gamma_{n c}$. Analogously, there exists a Hermitian metric $\gamma_{c}$ on $\mathbf{L}_{\lambda}$ invariant under $U_{\mathbb{R}}$ or its inverse image in the 2 -fold covering. As the quotient of two real algebraic metrics on a line bundle,

$$
f_{\lambda}=\frac{\gamma_{n c}}{\gamma_{c}}
$$

is a positive, real algebraic function on $S$. In the case of $\lambda=-2 \rho$, this function was first used in [S1] to study the Dolbeault cohomology of $G_{\mathbb{R}^{-}}$-equivariant line bundles.

7.10 Lemma. If $\lambda \in \Lambda+\rho$ satisfies the anti-dominance condition (7.3), some positive integral power $f_{\lambda}^{m}$ of the function $f_{\lambda}$ extends real algebraically to all of $X$, and this extension vanishes on the boundary of $S$. The image $\mu_{\lambda}\left(d \log f_{\lambda}\right)$ of $d \log f_{\lambda}$, viewed as a submanifold of $T^{*} S$, coincides with $\Omega(S, \lambda)$. More precisely,

$$
\mu_{\lambda}: d \log f_{\lambda} \longrightarrow \Omega(S, \lambda)
$$

is a real algebraic isomorphism which preserves or reverses orientation, depending on whether the integer $s$ is even or odd; here we orient $\Omega(S, \lambda)$ as in 7.8 and $d \log f_{\lambda} \cong S$ via the complex structure of $X$.

Before embarking on the proof - which is lengthy, though not difficult - we show how to deduce Theorem 3.8 in the current setting. We apply Lemma 3.19 with $C_{1}=d \log f_{\lambda}$, with $C_{2}=\mathrm{CC}\left(R j_{*} \mathbb{C}_{S}\right)$, and with

$$
\tilde{C}=\text { image of }(0,1) \times S \rightarrow T^{*} X, \quad(t, x) \mapsto t d \log f_{\lambda}(x) .
$$

We orient $C_{1}$ via $\left|C_{1}\right|=d \log f_{\lambda} \cong S$ and the complex structure on $S$, and orient $\tilde{C}$ by means of $|\tilde{C}| \cong(0,1) \times S$. Since $f_{\lambda}^{m}$ is real algebraic, so is $d \log f_{\lambda}$. Thus $C_{1}$ is a real algebraic cycle, and $\tilde{C}$ is a semi-algebraic chain. The support $\left|C_{1}\right|$ coincides with $\mu_{\lambda}^{-1}(\Omega(S, \lambda))$, so the values of $\mu_{\lambda}$ on $\left|C_{1}\right|$ lie in $\Omega(S, \lambda) \subset i \mathfrak{g}_{\mathbb{R}}^{*}$. It follows that the real part of $\mu$ remains bounded on $\left|C_{1}\right|$ and $|\tilde{C}|$. The open embedding theorem 4.2 of [SV4], coupled with [SV4, proposition 3.25], implies

$$
\partial \tilde{C}=d \log f_{\lambda}-\operatorname{CC}\left(R j_{*} \mathbb{C}_{S}\right)=C_{1}-C_{2} .
$$


We have verified the hypotheses of Lemma 3.19 in the present setting, hence

$$
\int_{C_{1}} \mu_{\lambda}^{*} \hat{\phi}\left(-\sigma+\pi^{*} \tau_{\lambda}\right)^{n}=\int_{C_{2}} \mu_{\lambda}^{*} \hat{\phi}\left(-\sigma+\pi^{*} \tau_{\lambda}\right)^{n} .
$$

Since $C_{1}=(-1)^{s} \mu_{\lambda}^{-1} \Omega(S, \lambda)$ by Lemma 7.10, taking into account Proposition 3.3, we find

$$
\begin{aligned}
\int_{\Omega(S, \lambda)} \hat{\phi} \sigma_{\lambda}^{n} & =\int_{\mu_{\lambda}^{-1} \Omega(S, \lambda)} \mu_{\lambda}^{*} \hat{\phi}\left(-\sigma+\pi^{*} \tau_{\lambda}\right)^{n} \\
& =(-1)^{s} \int_{\mathrm{CC}\left(R j_{*} \mathbb{C}_{S}\right)} \mu_{\lambda}^{*} \hat{\phi}\left(-\sigma+\pi^{*} \tau_{\lambda}\right)^{n},
\end{aligned}
$$

for every test function $\phi \in C_{c}^{\infty}\left(\mathfrak{g}_{\mathbb{R}}\right)$. We combine this with Theorems 7.6 and 7.8, and conclude Theorem 3.8 in the case of the discrete series:

7.15 Proposition. Under the hypotheses stated at the beginning of this section, the character formula 3.8 holds.

Proof of Lemma 7.10. The function $f_{\lambda}$ depends multiplicatively on the parameter $\lambda$; in particular, $f_{\lambda}^{m}=f_{m \lambda}$. Thus, replacing $\lambda$ by an appropriate positive integral multiple $m \lambda$, we may as well assume that the parameter $\lambda$ is integral even with respect to any particular finite quotient of $G$. This observation allows us to replace $G$ by $G / Z_{[G, G]}\left(Z_{[G, G]}=\right.$ center of the commutator subgroup of $\left.G\right)$. Because of (7.3) we can define

$$
V_{\lambda}=\text { irreducible } G \text {-module with lowest weight } \lambda \text {. }
$$

For $x \in X$, let $\mathfrak{n}_{x}=\left[\mathfrak{b}_{x}, \mathfrak{b}_{x}\right]$ denote the unipotent radical of the isotropy algebra at $x$. Because of our convention for ordering the roots, the space of $\mathfrak{n}_{x}$-invariants $V_{\lambda}^{\mathfrak{n}_{x}}$ is the lowest weight space for the action of any concrete Cartan subgroup $\mathfrak{c} \subset \mathfrak{b}_{x}$, $\mathfrak{c} \cong \mathfrak{b}_{x} / \mathfrak{n}_{x} \cong \mathfrak{h}$. As $x$ varies over $X$, the one-dimensional subspaces $V_{\lambda}^{\mathfrak{n}_{x}} \subset V_{\lambda}$ constitute a line bundle, and

$$
\mathbf{L}_{\lambda}=\text { line bundle with fiber } V_{\lambda}^{\mathfrak{n}_{x}} \text { at } x .
$$

In fact, $\mathcal{O}\left(\mathbf{L}_{\lambda}\right)$ is the Beilinson-Bernstein localization of $V_{\lambda}$ at $\lambda$. Since $U_{\mathbb{R}}$ is compact, there exists an essentially unique $U_{\mathbb{R}}$-invariant metric $h_{c}$ on $V_{\lambda}$. Renormalizing this metric, if necessary, we can make the identification

$$
\gamma_{c}=\text { restriction of } h_{c} \text { to the lines } V_{\lambda}^{\mathfrak{n}_{x}}
$$

via (7.17).

We need an analogous description of $\gamma_{n c}$. Let $\theta: \mathfrak{g} \rightarrow \mathfrak{g}$ denote the Cartan involution corresponding to the maximal compact subgroup $K_{\mathbb{R}} \subset G_{\mathbb{R}}$, and $\mathfrak{p}_{\mathbb{R}}$ the $(-1)$-eigenspace of $\theta$ in $\mathfrak{g}_{\mathbb{R}}$. Then

$$
G_{\mathbb{R}}=\exp \left(\mathfrak{p}_{\mathbb{R}}\right) \cdot K_{\mathbb{R}} \quad \text { (Cartan decomposition). }
$$

Since $K_{\mathbb{R}}$ contains the compact Cartan subgroup $T_{\mathbb{R}}$, the Cartan involution is inner; see, for example, [Hel]. Specifically, there exists

$$
r_{\theta} \in T_{\mathbb{R}} \cap[G, G] \text {, such that } \theta=\operatorname{Ad} r_{\theta} .
$$

Since we had made $[G, G]$ center free, this identity uniquely determines $r_{\theta}$. But $\theta^{-1}=\theta$, hence

$$
r_{\theta}=r_{\theta}^{-1} .
$$


We claim: the identity

$$
h_{n c}(u, v)=h_{c}\left(u, r_{\theta} v\right) \quad\left(u, v \in V_{\lambda}\right)
$$

defines a $G_{\mathbb{R}}$-invariant indefinite Hermitian form. Indeed,

$$
h_{n c}(v, u)=h_{c}\left(v, r_{\theta} u\right)=h_{c}\left(r_{\theta} v, u\right)=\overline{h_{c}\left(u, r_{\theta} v\right)}=\overline{h_{n c}(u, v)}
$$

because $r_{\theta}=r_{\theta}^{-1} \in T_{\mathbb{R}} \subset U_{\mathbb{R}}$. Given any $g \in G_{\mathbb{R}}$, we write $g=p k$ with $p \in \exp \left(\mathfrak{p}_{\mathbb{R}}\right)$ and $k \in K_{\mathbb{R}}$. The Lie algebra $\mathfrak{u}_{\mathbb{R}}$ of $U_{\mathbb{R}}$ contains $i \mathfrak{p}_{\mathbb{R}}-$ in fact, $\mathfrak{u}_{\mathbb{R}}=\mathfrak{k}_{\mathbb{R}} \oplus i \mathfrak{p}_{\mathbb{R}}[\mathrm{Hel}]$ - hence both $\mathfrak{p}_{\mathbb{R}}$ and $\exp \mathfrak{p}_{\mathbb{R}}$ operate on $V_{\lambda}$ by symmetric operators, relative to $h_{c}$. Thus

$$
\begin{aligned}
h_{n c}(g u, g v) & =h_{c}\left(p k u, r_{\theta} p k v\right)=h_{c}\left(p k u, r_{\theta} p r_{\theta}^{-1} r_{\theta} k v\right)=h_{c}\left(p k u, p^{-1} r_{\theta} k v\right) \\
& =h_{c}\left(k u, p p^{-1} r_{\theta} k v\right)=h_{c}\left(u, k^{-1} r_{\theta} k r_{\theta}^{-1} r_{\theta} v\right)=h_{c}\left(u, k^{-1} k r_{\theta} v\right) \\
& =h_{c}\left(u, r_{\theta} v\right)=h_{n c}(u, v) .
\end{aligned}
$$

This establishes our claim. The Cartan involution preserves the root space decomposition

$$
\mathfrak{g}=\mathfrak{t} \oplus\left(\oplus_{\alpha \in \Phi} \mathfrak{g}^{\alpha}\right)
$$

and each root space is contained either in $\mathfrak{k}$ or in $\mathfrak{p}=\mathfrak{k}^{\perp}$, hence $e^{\alpha}\left(r_{\theta}\right)= \pm 1$. Since $G$ is center free, the weights of $V_{\lambda}$ lie in the root lattice. We conclude that $r_{\theta}$ acts as multiplication by \pm 1 on each $\mathfrak{t}$-weight space in $V_{\lambda}$. In particular, the $G_{\mathbb{R}}$-invariant indefinite Hermitian form $h_{n c}$ is either strictly positive or strictly negative on all the lines $V_{\lambda}^{\mathfrak{n}_{x}}, x \in S$. Renormalizing $h_{n c}$ by an appropriate positive or negative factor, we get the description

$$
\gamma_{n c}=\text { restriction of } h_{n c} \text { to the lines } V_{\lambda}^{\mathfrak{n}_{x}}, x \in S,
$$

of $\gamma_{n c}$ analogous to (7.18).

The lines $V_{\lambda}^{\mathfrak{n}_{x}}$ vary algebraically with $x$, and $h_{c}$ is positive definite for every $x \in X$. It follows that the ratio $h_{n c} / h_{c}$ of the two metrics on the lines $V_{\lambda}^{\mathfrak{n}_{x}}$ is a globally defined, real algebraic function on $X$, which agrees with $f_{\lambda}$ on $S$. Now let $x$ be a point in the boundary of $S$; we claim:

$$
h_{n c} \text { vanishes on } V_{\lambda}^{\mathfrak{n}_{x}} \text {. }
$$

Since $h_{n c}$ is $G_{\mathbb{R}}$-invariant, we may replace $x$ by any $G_{\mathbb{R}}$-translate. In this way we can arrange that the point $x$ is fixed by a $\theta$-stable Cartan subalgebra $\mathfrak{c}_{\mathbb{R}}$ of $\mathfrak{g}_{\mathbb{R}}[\mathrm{Ma}]$. The corresponding Cartan subgroup $C_{\mathbb{R}} \subset G_{\mathbb{R}}$ is noncompact since otherwise $x$ would have to lie in an open $G_{\mathbb{R}^{-}}$-orbit, and consequently $\theta$ does not act as the identity on the complexified Cartan subalgebra $\mathfrak{c} \subset \mathfrak{g}$. Both $V_{\lambda}^{\mathfrak{n}_{x}}$ and $r_{\theta} V_{\lambda}^{\mathfrak{n}_{x}}$ are weight spaces of $\mathfrak{c}$, and the corresponding weights are necessarily distinct because the lowest weight of $V_{\lambda}$ is regular, and $\theta=\operatorname{Ad} r_{\theta}$ normalizes $\mathfrak{c}$ and acts as a nontrivial element of the Weyl group, as we have just seen. On the other hand, as a $\theta$-stable, $\mathfrak{g}_{\mathbb{R}}$-real Cartan subalgebra, $\mathfrak{c}$ is also $\mathfrak{u}_{\mathbb{R}}$-real. This makes the $\mathfrak{c}$-weight space decomposition of $V_{\lambda}$ perpendicular with respect to $h_{c}$. The $h_{c}$-perpendicularity of the two lines $V_{\lambda}^{\mathfrak{n}_{x}}$ and $r_{\theta} V_{\lambda}^{\mathfrak{n}_{x}}$ implies (7.23), which in turn implies the vanishing at $x$ of the natural extension of $f_{\lambda}$ to $X$. This establishes the first part of the lemma.

Both $S$ and $\Omega(S, \lambda)$ are homogenous spaces for $G_{\mathbb{R}}$, the former with isotropy subgroup $T_{\mathbb{R}}$ at $x_{0}$, the latter with isotropy subgroup $T_{\mathbb{R}}$ at $\tau_{x_{0}}^{*} \lambda$. To see this in the case of $S$, we recall (6.6a) and note that $\mathfrak{n}_{x_{0}} \cap \overline{\mathfrak{n}}_{x_{0}}=0\left(\overline{\mathfrak{n}}_{x_{0}}=\right.$ complex conjugate of $\mathfrak{n}_{x_{0}}$ with respect to $\left.\mathfrak{g}_{\mathbb{R}}\right)$ since all roots are imaginary on the Lie algebra of the 
compact Cartan $T_{\mathbb{R}}$. In the case of $\Omega(S, \lambda)$, we appeal to the regularity of $\tau_{x_{0}}^{*} \lambda$ and the equality $T_{\mathbb{R}}=G_{\mathbb{R}} \cap T$. We conclude that

$$
I: S \stackrel{\sim}{\longrightarrow} \Omega(S, \lambda), \quad I\left(g x_{0}\right)=\operatorname{Ad} g\left(\tau_{x_{0}}^{*} \lambda\right) \text { if } g \in G_{\mathbb{R}},
$$

is a diffeomorphism. To calculate $\mu_{\lambda}^{-1} \circ I$, we write

$$
\operatorname{Ad} g\left(\tau_{x_{0}}^{*} \lambda\right)=I\left(g x_{0}\right)=\mu_{\lambda}(\tilde{x}, \xi) \quad \text { with } \tilde{x} \in X \text { and } \xi \in T_{\tilde{x}}^{*} X .
$$

We identify $\mathfrak{h}^{*} \cong\left(\mathfrak{b}_{\tilde{x}} / \mathfrak{n}_{\tilde{x}}\right)^{*} \cong \mathfrak{n}_{\tilde{x}}^{\perp} / \mathfrak{b}_{\tilde{x}}^{\perp}$. Then, by definition of $\mu_{\lambda}$,

$$
\mu_{\lambda}(\tilde{x}, \xi) \in \mathfrak{n}_{\tilde{x}}^{\perp}, \quad \mu_{\lambda}(\tilde{x}, \xi) \equiv \lambda \bmod \mathfrak{b}_{\tilde{x}}^{\perp} .
$$

On the other hand,

$$
\operatorname{Ad} g\left(\tau_{x_{0}}^{*} \lambda\right) \in \mathfrak{n}_{g x_{0}}^{\perp}, \quad \operatorname{Ad} g\left(\tau_{x_{0}}^{*} \lambda\right) \equiv \lambda \bmod \mathfrak{b}_{g x_{0}}^{\perp} .
$$

Since $\lambda$ is regular, (7.25a-c) imply $g x_{0}=\tilde{x}$. We choose $u \in U_{\mathbb{R}}$ so that

$$
g x_{0}=\tilde{x}=u x_{0} .
$$

The definition of $\mu_{\lambda}$ gives the identity

$$
\mu_{\lambda}(\tilde{x}, \xi)=\operatorname{Ad} u\left(\tau_{x_{0}}^{*} \lambda\right)+\mu(\xi) .
$$

In effect, we have calculated $\mu_{\lambda}^{-1} \circ I$ :

$$
\left(\mu_{\lambda}^{-1} \circ I\right)\left(g x_{0}\right)=\left(g x_{0}, \xi\right), \text { with } \mu(\xi)=\operatorname{Ad} g\left(\tau_{x_{0}}^{*} \lambda\right)-\operatorname{Ad} u\left(\tau_{x_{0}}^{*} \lambda\right) ;
$$

here $u$ is determined by (7.26a), uniquely up to right multiplication by an element of $T_{\mathbb{R}}$. In particular, when we compose $\mu_{\lambda}^{-1} \circ I$ with the projection $\pi: T^{*} X \rightarrow X$ we obtain the identity on $S$. At this point, the remaining assertions of the lemma come down to

$$
\mu\left(\left.d \log f_{\lambda}\right|_{g x_{0}}\right)=\operatorname{Ad} g\left(\tau_{x_{0}}^{*} \lambda\right)-\operatorname{Ad} u\left(\tau_{x_{0}}^{*} \lambda\right),
$$

whenever $g \in G_{\mathbb{R}}, u \in U_{\mathbb{R}}$ and $g x_{0}=u x_{0}$ as before, and

(7.27b) $I: S \stackrel{\sim}{\longrightarrow} \Omega(S, \lambda)$ is orientation preserving if and only if $s$ is even

when $S$ and $\Omega(S, \lambda)$ are oriented as in the statement of the lemma.

We deal with (7.27a) first. In the statement of the lemma, as in (7.27a), we regard the differential $d \log f_{\lambda}$ as a real algebraic section of the cotangent bundle; this involves an explicit isomorphism $T^{*} X \cong T^{*} X^{\mathbb{R}}$, which we normalize as in [KSa, Chapter 11]. Hence, by the definition of the moment map,

$$
\left.\frac{d}{d t} \log f_{\lambda}\left(\exp (t Z) g x_{0}\right)\right|_{t=0}=2 \operatorname{Re}\left\langle\mu\left(\left.d \log f_{\lambda}\right|_{g x_{0}}\right), Z\right\rangle,
$$

for every $Z \in \mathfrak{g}$. Let $\ell$ be a generator of $V_{\lambda}^{\mathfrak{n}_{g x_{0}}}$. Then, by (7.18) and (7.22),

$$
\begin{aligned}
& \left.\frac{d}{d t} \log f_{\lambda}\left(\exp (t Z) g x_{0}\right)\right|_{t=0} \\
& \quad=\frac{h_{n c}(Z \ell, \ell)+h_{n c}(\ell, Z \ell)}{h_{n c}(\ell, \ell)}-\frac{h_{c}(Z \ell, \ell)+h_{c}(\ell, Z \ell)}{h_{c}(\ell, \ell)} .
\end{aligned}
$$

Both summands on the right are homogenous of degree zero in $\ell$, so we may use two different generators of $V_{\lambda}^{\mathfrak{n}_{g x_{0}}}$ in the two summands. Let us use $\ell=g \ell_{0}$ in the 
first instance, with $\ell_{0} \in V_{\lambda}^{\mathfrak{n}_{x_{0}}}$, and $\ell=u \ell_{0}$ in the second instance:

$$
\begin{aligned}
& \left.\frac{d}{d t} \log f_{\lambda}\left(\exp (t Z) g x_{0}\right)\right|_{t=0} \\
& \quad=\frac{h_{n c}\left(Z g \ell_{0}, g \ell_{0}\right)+h_{n c}\left(g \ell_{0}, Z g \ell_{0}\right)}{h_{n c}\left(\ell_{0}, \ell_{0}\right)}-\frac{h_{c}\left(Z u \ell_{0}, u \ell_{0}\right)+h_{c}\left(u \ell_{0}, Z u \ell_{0}\right)}{h_{c}\left(\ell_{0}, \ell_{0}\right)} .
\end{aligned}
$$

Note that $h_{n c}\left(Z g \ell_{0}, g \ell_{0}\right)=h_{n c}\left(\left(\operatorname{Ad} g^{-1} Z\right) \ell_{0}, \ell_{0}\right)$ depends only on the $\mathfrak{t}$-component of $\operatorname{Ad}^{-1} Z$ in the decomposition $\mathfrak{g}=\mathfrak{t} \oplus[\mathfrak{t}, \mathfrak{g}]$, since the $\mathfrak{t}$-weight spaces in $V_{\lambda}$ are $h_{n c}$-perpendicular. On the other hand, $Y \ell_{0}=\left\langle\tau_{x_{0}}^{*} \lambda, Y\right\rangle \ell_{0}$ if $Y \in \mathfrak{t}$. Thus

$$
\frac{h_{n c}\left(Z g \ell_{0}, g \ell_{0}\right)}{h_{n c}\left(\ell_{0}, \ell_{0}\right)}=\frac{h_{n c}\left(\left(\operatorname{Ad} g^{-1} Z\right) \ell_{0}, \ell_{0}\right)}{h_{n c}\left(\ell_{0}, \ell_{0}\right)}=\left\langle\tau_{x_{0}}^{*} \lambda,\left(\operatorname{Ad} g^{-1} Z\right)\right\rangle .
$$

Arguing similarly in the case of the other terms on the right of (7.30), we find

$$
\begin{aligned}
\left.\frac{d}{d t} \log f_{\lambda}\left(\exp (t Z) g x_{0}\right)\right|_{t=0} & =2 \operatorname{Re}\left\langle\tau_{x_{0}}^{*} \lambda,\left(\operatorname{Ad} g^{-1} Z\right)-\left(\operatorname{Ad} u^{-1} Z\right)\right\rangle \\
& =2 \operatorname{Re}\left\langle\operatorname{Ad} g\left(\tau_{x_{0}}^{*} \lambda\right)-\operatorname{Ad} u\left(\tau_{x_{0}}^{*} \lambda\right), Z\right\rangle .
\end{aligned}
$$

Taken together, (7.28) and (7.31) imply (7.27a).

We verify the orientation statement $(7.27 \mathrm{~b})$ by reducing it to the special cases of $G_{\mathbb{R}}=S U(1,1)$ and $G_{\mathbb{R}}=S U(2)$. To begin with, we need to establish the statement only at a single point - specifically, at the fixed point $x_{0} \in S$ for $T_{\mathbb{R}}$. We identify the holomorphic tangent space $T_{\lambda} \Omega_{\lambda}$ of the complex orbit at $\lambda$ with

$$
\mathfrak{g} / \mathfrak{t} \cong[\mathfrak{t}, \mathfrak{g}]=\bigoplus_{\alpha \in \Phi(\mathfrak{g}, \mathfrak{t})} \mathfrak{g}^{\alpha} .
$$

There are analogous descriptions for the real tangent space $T_{\lambda} \Omega(S, \lambda)$,

$$
\mathfrak{g}_{\mathbb{R}} / \mathfrak{t}_{\mathbb{R}} \cong T_{\lambda} \Omega(S, \lambda) \hookrightarrow \mathbb{C} \otimes_{\mathbb{R}} T_{\lambda} \Omega(S, \lambda) \cong \mathfrak{g} / \mathfrak{t},
$$

and of the real tangent space $T_{x_{0}} S^{\mathbb{R}}$ of the open orbit $S \subset X$ considered as a real manifold,

$$
\mathfrak{g}_{\mathbb{R}} / \mathfrak{t}_{\mathbb{R}} \cong T_{x_{0}} S^{\mathbb{R}} \hookrightarrow \mathbb{C} \otimes_{\mathbb{R}} T_{x_{0}} S^{\mathbb{R}} \cong \mathfrak{g} / \mathfrak{t}
$$

Via these identifications, the differential of the map $I$ at $x_{0}$ becomes the identity, and the symplectic form $\sigma_{\lambda}$ is given by the formula

$$
\sigma_{\lambda}\left(Z_{1}, Z_{2}\right)=\left\langle\lambda,\left[Z_{1}, Z_{2}\right]\right\rangle, \quad Z_{1}, Z_{2} \in[\mathfrak{t}, \mathfrak{g}] .
$$

Note that $\langle\lambda,[\mathfrak{t}, \mathfrak{g}]\rangle=0$, hence

$$
\sigma_{\lambda}\left(\mathfrak{g}^{\alpha}, \mathfrak{g}^{\beta}\right)=0 \quad \text { unless } \alpha+\beta=0 .
$$

The isomorphism $\tau_{x_{0}}^{*}: \Phi \cong \Phi(\mathfrak{g}, \mathfrak{t})$ induces a positive root system $\Phi^{+}(\mathfrak{g}, \mathfrak{t})$. Each root $\alpha \in \Phi(\mathfrak{g}, \mathfrak{t})$ is either compact or noncompact, in the sense that the subalgebra

$$
\mathfrak{g}_{\alpha}=\mathfrak{g}^{\alpha} \oplus \mathfrak{g}^{-\alpha} \oplus\left[\mathfrak{g}^{\alpha}, \mathfrak{g}^{-\alpha}\right] \cong \mathfrak{s l}(2, \mathbb{C})
$$

of $\mathfrak{g}$ intersects $\mathfrak{g}_{\mathbb{R}}$ in a copy of either $\mathfrak{s u}(2)$ or $\mathfrak{s u}(1,1)$. Let $G_{\alpha}$ denote the connected subgroup of $G$ with Lie algebra $\mathfrak{g}_{\alpha}$, and $G_{\mathbb{R}, \alpha}$ the real form $G_{\mathbb{R}} \cap G_{\alpha}$. Then $G_{\mathbb{R}, \alpha} \cdot x_{0}$, the $G_{\mathbb{R}, \alpha^{-}}$-orbit of $x_{0}$ in $S$, is isomorphic as homogenous space to $\mathbb{P}^{1}$ if $\alpha$ is compact, or to $\Delta=$ unit disc if $\alpha$ is noncompact. The three spaces $\Omega_{\lambda}, \Omega(S, \lambda)$, and $S$ split locally into products of the orbits $G_{\alpha} \cdot \lambda, G_{\mathbb{R}, \alpha} \cdot \lambda$, and $G_{\mathbb{R}, \alpha} \cdot x_{0}$, respectively, corresponding to the various positive roots $\alpha \in \Phi^{+}(\mathfrak{g}, \mathfrak{t})$. These splittings are compatible with the definition (7.24) of $I$ and with symplectic form (7.34) - note: $\lambda$ induces an anti-dominant, regular weight for each $\mathfrak{g}_{\alpha}^{*}$ by restriction. The integer 
$s$ equals the number of positive compact roots. These considerations reduce the statement (7.27b) to the special cases of $G_{\mathbb{R}}=S U(2)$ and $G_{\mathbb{R}}=S U(1,1)$. In these two cases, it can be verified by direct calculation. This completes the proof of Lemma 7.10.

We conclude this section with the verification of the fixed point formulas 5.12, $5.24,5.27$ in the case of the discrete series - i.e., under the hypotheses enunciated at the beginning of this section. Kashiwara already verified his conjecture for the discrete series (with anti-dominant $\lambda$, as in our current setting). Our argument differs from his only by replacing a reference to a lemma of $[\mathrm{OM}]$ by a short calculation.

Discrete series characters - both on the group and the Lie algebra - are uniquely determined, among all invariant eigendistributions, by a) their restriction to the elliptic set, and b) being tempered [HC5]. The fixed point formalism discussed in $\S 4$ does produce invariant eigendistributions. Thus we only need to verify those properties of the coefficients $c_{g, x}$ and $d_{E, x}$, corresponding to $\mathcal{F}=R j_{*} \mathbb{C}_{S}$, which embody the properties a) and b).

Because of 7.1, a globally well defined section $e^{\lambda-\rho}$ of $\mathbb{C}_{\lambda}$ exists on the universal Cartan $H$, and thus on all of the spaces mapping naturally to $H$. Harish-Chandra's formula for the discrete series characters on the elliptic set can be stated as follows:

$$
\Theta(S, \lambda)(g)=(-1)^{s} \sum_{x \in X^{g} \cap S} \frac{e^{\lambda-\rho}(g, x)}{\prod_{\alpha \in \Phi^{+}}\left(1-e^{-\alpha}\right)(g, x)} \quad(g \text { regular elliptic); }
$$

the appearance of $(-1)^{s}$, as opposed to $(-1)^{q}$ in Harish-Chandra's original formula, is caused by anti-dominance of $\lambda$ : in the definition of the Weyl denominator, HarishChandra uses the positive root system which makes the parameter $\lambda$ dominant. The alternating nature of the summation in Harish-Chandra's formula translates into the dependence of the denominator on the fixed point $x$. We now suppose that $g$ lies in the compact Cartan subgroup $T_{\mathbb{R}}$ chosen at the beginning of the section, and write $t$ instead of $g$ for a generic element of $T_{\mathbb{R}}^{\prime}$, as in (5.22)-(5.25). There are no real roots on $\mathfrak{t}_{\mathbb{R}}$, so we can choose $\Psi^{\prime}=\Phi^{+}$in the definition of $\mathfrak{n}^{\prime}(t, x)$. Hence $N^{\prime}(t, x)=N^{+}(t, x)$ is an open Schubert cell containing the fixed point $x$. In particular,

$$
\begin{array}{r}
\chi\left(\mathcal{H}_{N^{\prime}(t, x)}^{*}\left(\mathbb{D} R j_{*} \mathbb{C}_{S}\right)_{x}\right)=\chi\left(\mathcal{H}_{N^{\prime}(t, x)}^{*}\left(j ! \mathbb{D}_{S}\right)_{x}\right) \\
=\chi\left(\mathcal{H}^{*}\left(j_{!} \mathbb{C}_{S}[2 n]\right)_{x}\right)= \begin{cases}1 & \text { if } x \in S, \\
0 & \text { if } x \notin S .\end{cases}
\end{array}
$$

This, in conjunction with (5.25) and 7.6, establishes a).

The temperedness condition b) comes down to the vanishing of certain coefficients $c_{t, x}$ corresponding to regular semisimple $t$. We now deviate from the earlier notation of this section: to be consistent with $\S 5, T_{\mathbb{R}}$ will be an arbitrary Cartan subgroup, $t$ a regular element of $T_{\mathbb{R}}$, and $x$ a fixed point of $t$. Nonzero terms in the local expression ${ }^{11}$ (4.3) for $\Theta=(-1)^{s} \Theta\left(R j_{*} \mathbb{C}_{S}\right)$ violate temperedness precisely when they are indexed by a pair $(t, x) \in \tilde{G}_{\mathbb{R}}$ with $\left|e^{\lambda_{x}}(t)\right|>1$; note: $e^{\rho}$ is defined up to sign on $\tilde{G}$, and hence so is $e^{\lambda}=e^{\lambda-\rho} e^{\rho}$. Hence, in view of (5.25), the condition b) is equivalent to

$$
\chi\left(\mathcal{H}_{\{x\}}^{*}\left(\left.\left(\mathbb{D} R j_{*} \mathbb{C}_{S}\right)\right|_{N^{\prime \prime}(t, x)}\right)\right)=0 \quad \text { if } \quad\left|e^{\lambda_{x}}(t)\right|>1 .
$$

\footnotetext{
${ }^{11} \mathrm{With} \zeta=0$, and re-expressed in terms of the constants $d_{t, x}$ of (5.25).
} 
The definition (5.17) of $N^{\prime \prime}(t, x)$ depends on the choice of the subset $\Psi^{\prime \prime}$ in $\Phi^{+}$. The conditions (5.15) allow us to pick

$$
\Psi^{\prime \prime}=\left\{\alpha \in \Phi^{+} \mid \alpha \text { is real valued on } \mathfrak{t}_{\mathbb{R}} \text { and } e^{\alpha}(t)>1\right\} .
$$

To verify the temperedness condition b), we only need to show that

$$
N^{\prime \prime}(t, x) \cap S=\emptyset \quad \text { if } \quad\left|e^{\lambda_{x}}(t)\right|>1,
$$

since then $\left.j ! \mathbb{C}_{S}\right|_{N^{\prime \prime}(t, x)}=0$, hence

$$
\mathcal{H}_{\{x\}}^{*}\left(\left.\left(\mathbb{D} R j_{*} \mathbb{C}_{S}\right)\right|_{N^{\prime \prime}(t, x)}\right)=\mathcal{H}_{\{x\}}^{*}\left(\left.\left(j ! \mathbb{C}_{S}[2 n]\right)\right|_{N^{\prime \prime}(t, x)}\right)=0 .
$$

The condition (7.40) holds vacuously when the Cartan subgroup $T_{\mathbb{R}}$ containing $t$ is compact. Let us assume, then, that $T_{\mathbb{R}}$ is a noncompact Cartan subgroup of a group $G_{\mathbb{R}}$ which does contain some compact Cartan. In this situation the fixed points of $t \in T_{\mathbb{R}}^{\prime}$ cannot lie in open $G_{\mathbb{R}}$-orbits. Thus the nonvacuous case of (7.40) is a consequence of:

7.41 Lemma. Let $x \in X$ be a point not lying in any open $G_{\mathbb{R}}$-orbit and $t \in G_{\mathbb{R}} a$ regular semisimple element fixing $x$, such that $\left|e^{\lambda_{x}}(t)\right| \geq 1$. Then $N^{\prime \prime}(t, x) \cap S=\emptyset$, provided $\Psi^{\prime \prime}$ is chosen as in (7.39).

Proof. We replace $\lambda$ by a suitable positive integral multiple so that the function $f_{\lambda}$ of Lemma 7.10 extends to all of $X$. Let $\zeta \in \mathfrak{n}^{\prime \prime}(t, x)$ be such that $(\exp \zeta) x \in S$. Because of the choice of $\Psi$, this implies $\operatorname{Ad}\left(t^{-n}\right) \zeta \rightarrow 0$ as $n \rightarrow+\infty$. We now use the notation of the proof of 7.10 :

$$
\begin{aligned}
(\exp \zeta) x \in S & \Longrightarrow f_{\lambda}((\exp \zeta) x) \neq 0 \\
& \Longrightarrow h_{n c} \neq 0 \text { on } V_{\lambda}^{\mathfrak{n}_{\exp \zeta x}}=\exp \zeta V_{\lambda}^{\mathfrak{n}_{x}} \\
& \Longrightarrow h_{n c}\left(\exp \zeta v_{0}, \exp \zeta v_{0}\right) \neq 0 \text { for } v_{0} \neq 0 \text { in } V_{\lambda}^{\mathfrak{n}_{x}} \\
& \Longrightarrow h_{n c}\left(t^{-n} \exp \zeta v_{0}, t^{-n} \exp \zeta v_{0}\right) \neq 0 ;
\end{aligned}
$$

here we are using the $G_{\mathbb{R}^{-}}$invariance of $h_{n c}$. But

$$
\begin{aligned}
& h_{n c}\left(t^{-n} \exp \zeta v_{0}, t^{-n} \exp \zeta v_{0}\right) \\
& =h_{n c}\left(\exp \left(\operatorname{Ad}\left(t^{-n}\right) \zeta\right) t^{-n} v_{0}, \exp \left(\operatorname{Ad}\left(t^{-n}\right) \zeta\right) t^{-n} v_{0}\right) \\
& =\left|e^{\lambda_{x}}\left(t^{-n}\right)\right|^{2} h_{n c}\left(\exp \left(\operatorname{Ad}\left(t^{-n}\right) \zeta\right) v_{0}, \exp \left(\operatorname{Ad}\left(t^{-n}\right) \zeta\right) v_{0}\right)
\end{aligned}
$$

Since $\operatorname{Ad}\left(t^{-n}\right) \zeta \rightarrow 0$ and $h_{n c}\left(v_{0}, v_{0}\right)=0$ - recall: $x$ does not lie in an open orbit, hence $h_{n c}=0$ on $V_{\lambda}^{\mathfrak{n}_{x}}$ - this is possible only if $\left|e^{\lambda_{x}}\left(t^{-n}\right)\right| \rightarrow \infty$ as $n \rightarrow+\infty$. In other words, the hypothesis $(\exp \zeta) x \in S$ forces $\left|e^{\lambda_{x}}(t)\right|<1$.

The two statements (7.37) and (7.40) now imply the fixed point formula 5.24, and hence also 5.12, for the discrete series characters $\Theta(S, \lambda)$. In the case of the characters $\theta(S, \lambda)$ on the Lie algebra, the argument is virtually identical. Thus:

7.42 Proposition. Under the hypotheses stated at the beginning of this section, the character formulas 5.12, 5.24, and 5.27 hold. 


\section{Coherent COntinuation}

Both of our character formulas are compatible with the representation-theoretic process of coherent continuation [S3]. In the case of the integral formula 3.8 this is not so easy to see a priori, though it follows easily from the formula once it has been proved. In this section, we shall use the process of coherent continuation to establish our character formulas for the coherent continuations of all discrete series characters. In effect, we shall remove the positivity condition (7.3) as hypothesis from Propositions 7.15 and 7.42 .

Let us recall the notion of coherent continuation. A family of invariant eigendistributions $\Theta(\lambda)$ parametrized by a coset $\lambda_{0}+\Lambda$ of the weight lattice $\Lambda$ depends coherently on $\lambda$ provided

a) $\mathcal{Z}(\mathfrak{g})$ operates on $\Theta(\lambda)$ according to the infinitesimal character $\chi_{\lambda}$ for every $\lambda \in \lambda_{0}+\Lambda$;

b) the coefficients $c_{g, x}=c_{g, x}(\lambda)$ in the local expressions (4.3) for the $\Theta(\lambda)$ satisfy $c_{g, x}(\lambda)=e^{\lambda-\lambda_{0}}(g) c_{g, x}\left(\lambda_{0}\right)$.

Note that $\lambda-\lambda_{0}$ is an integral weight, so $e^{\lambda-\lambda_{0}}(g)$ is well defined. The condition b) can be stated differently, as follows. Let $\phi$ be a finite-dimensional character. Then

$$
\phi=\sum_{\mu \in \Lambda} n_{\mu}(\phi) e^{\mu}
$$

with only finitely many $n_{\mu}(\phi) \neq 0$. On any concrete Cartan, this formula has an obvious meaning; since it is symmetric under the Weyl group, it makes sense also to state it in universal terms, as above. If the family $\Theta(\lambda)$ satisfies (8.1a), the second condition $(8.1 \mathrm{~b})$ is equivalent to

$$
\phi \theta(\lambda)=\sum_{\mu \in \Lambda} n_{\mu}(\phi) \Theta(\lambda+\mu),
$$

for every $\lambda \in \lambda_{0}+\Lambda$ and every finite-dimensional character $\phi$ [S3].

Let $\Theta(\lambda)$ be a coherent family parametrized by $\lambda_{0}+\Lambda$. If $\lambda_{0}$ is regular, the single member $\Theta\left(\lambda_{0}\right)$ of the family determines all the others - this follows immediately from (8.1b), coupled with the uniqueness of the coefficients $c_{g, x}(\lambda)$ in the case of regular parameter $\lambda$. Thus:

8.4 Observation. Two coherent families parametrized by the same coset $\lambda_{0}+\Lambda$ coincide as soon as they agree at a single regular parameter $\lambda \in \lambda_{0}+\Lambda$.

In the definition (2.17), we have attached the invariant eigendistribution $\Theta(\mathcal{F})$ to $\mathcal{F} \in \mathrm{D}_{G_{\mathbb{R}}}(X)_{-\lambda}$ and the datum of a specific $\lambda$, though the dependence on $\lambda$ does not come out in the notation - recall that $\mathrm{D}_{G_{\mathbb{R}}}(X)_{-\lambda}$ depends only on the image of $\lambda$ in $\mathfrak{h}^{*} / \Lambda$. In the present section, we need to make the dependence on $\lambda$ explicit. Thus, for $\lambda \in \lambda_{0}+\Lambda$ and $\mathcal{F} \in \mathrm{D}_{G_{\mathbb{R}}}(X)_{-\lambda_{0}}$ we shall write $\Theta_{\lambda}(\mathcal{F})$ for the invariant eigendistribution (2.17) corresponding to the representative $\lambda$ of the coset $\lambda_{0}+\Lambda$.

8.5 Theorem. The invariant eigendistributions $\Theta_{\lambda}(\mathcal{F}), \lambda \in \lambda_{0}+\Lambda$, constitute a coherent family.

This is a standard fact. As specific reference let us mention [SW], where the coherence is proved for standard sheaves; that is enough, of course, in view of 
Lemma 6.4. Alternatively, one can use the corresponding fact about the BeilinsonBernstein construction - see [Mi], for example - and carry it over to our setting via the main result in $[\mathrm{KSd}]$.

Kashiwara's fixed point formalism, as discussed in $\S 5$, associates another eigendistribution to the datum of a $\lambda \in \mathfrak{h}^{*}$ and $\mathcal{F} \in \mathrm{D}_{G_{\mathbb{R}}}(X)_{-\lambda}$, thus a family $\tilde{\Theta}_{\lambda}(\mathcal{F})$ parametrized by the $\lambda$-coset of the weight lattice. In effect, $\tilde{\Theta}_{\lambda}(\mathcal{F})$ is the invariant eigendistribution described by the right hand side of the equation in Theorem 5.12.

8.6 Lemma. The family $\tilde{\Theta}_{\lambda}(\mathcal{F})$ is coherent

Proof. The character cycle $c(\mathcal{F})$ is a cycle of top degree with values in the local system $\mathbb{C}_{-\lambda}$, which is completely determined by the section $c:(g, x) \mapsto c_{g, x}$ of the dual local system $\mathbb{C}_{\lambda}$ over $\tilde{G}_{\mathbb{R}}^{\prime}-$ cf. (4.12). The coherence condition (8.1b) for the family $\tilde{\Theta}_{\lambda}(\mathcal{F})$ is equivalent to the following statement: when $\lambda$ is replaced by the translate $\lambda+\mu$ by some $\mu \in \Lambda$, the section $c:(g, x) \mapsto c_{g, x}(\lambda)$ gets multiplied by $e^{\mu}$. This multiplicative behavior of $c$ is clear from the construction; the dependence of the character cycle on the specific parameter $\lambda$, rather than on the coset $\lambda+\Lambda$, appears at exactly one point, the passage from $\phi^{\prime}$ to $\phi^{\prime \prime}$ in (5.7)-(5.8) which is multiplicative in the sense mentioned earlier.

The general principle 8.4, Theorem 8.5, and Lemma 8.6 allow us to remove the positivity hypothesis (7.3) in Proposition 7.42. For later reference, we state

8.7 Proposition. The character formulas 5.12, 5.24, and 5.27 are satisfied by every standard sheaf associated to an open $G_{\mathbb{R}}$-orbit, provided the group $G_{\mathbb{R}}$ contains a compact Cartan subgroup.

According to 8.4 the invariant eigendistributions (7.2) constitute a coherent family $\Theta_{\lambda}\left(R j_{*} \mathbb{C}_{S}\right)$, parametrized by $\lambda \in \Lambda-\rho$. Let us write $\theta_{\lambda}\left(R j_{*} \mathbb{C}_{S}\right)$ for the corresponding family on the Lie algebra. The conditions (8.1) and (8.2) have obvious analogues on the Lie algebra. Thus it makes sense to talk about coherent families of invariant eigendistributions on the Lie algebra. The coherence of $\Theta_{\lambda}\left(R j_{*} \mathbb{C}_{S}\right)$ implies coherence also for the family $\theta_{\lambda}\left(R j_{*} \mathbb{C}_{S}\right)$. Unlike the $\Theta_{\lambda}\left(R j_{*} \mathbb{C}_{S}\right)$, which are defined only for $\lambda \in \Lambda-\rho$, the $\theta_{\lambda}\left(R j_{*} \mathbb{C}_{S}\right)$ can be given meaning for any $\lambda \in \mathbb{R} \otimes_{\mathbb{Z}} \Lambda$, as follows. The integral on the left in (7.14) converges for every regular $\lambda \in \mathbb{R} \otimes_{\mathbb{Z}} \Lambda$, and thus defines a family of invariant eigendistributions

$$
\phi \longmapsto \int_{\Omega(S, \lambda)} \hat{\phi} \sigma_{\lambda}^{n} \quad\left(\phi \in C_{c}^{\infty}\left(\mathfrak{g}_{\mathbb{R}}\right)\right),
$$

parametrized by the regular set in $\mathbb{R} \otimes_{\mathbb{Z}} \Lambda$. According to Rossmann's Theorem 7.8 , this distribution coincides with the discrete series character $\theta_{\lambda}\left(R j_{*} \mathbb{C}_{S}\right)$ when $\lambda \in \Lambda-\rho$ satisfies the anti-dominance condition (7.3):

$$
\begin{array}{r}
\int_{\mathfrak{g}_{\mathbb{R}}} \phi \theta_{\lambda}\left(R j_{*} \mathbb{C}_{S}\right) d x=\frac{1}{(2 \pi i)^{n} n !} \int_{\Omega(S, \lambda)} \hat{\phi} \sigma_{\lambda}^{n} \\
\text { for } \lambda \in \Lambda-\rho \text { regular anti-dominant. }
\end{array}
$$

Since the right hand side is well defined for any regular anti-dominant $\lambda \in \mathbb{R} \otimes_{\mathbb{Z}} \Lambda$, so is the family $\theta_{\lambda}\left(R j_{*} \mathbb{C}_{S}\right)$. At this point, then, the family is well defined for all $\lambda \in \mathbb{R} \otimes_{\mathbb{Z}} \Lambda$ which either lie in $\Lambda-\rho$, or are anti-dominant regular. The following result is due to Harish-Chandra [HC5, lemma 32]. 
8.10 Lemma. The coefficients $d_{E, x}$ in the local expressions (4.4) for the invariant eigendistributions (8.8) depend only on the Weyl chamber in which $\lambda$ lies.

Because of the equality (8.9), we conclude that the coefficients in the local expressions for the $\theta_{\lambda}\left(R j_{*} \mathbb{C}_{S}\right)$ are also independent of $\lambda$. Thus we can coherently continue the $\theta_{\lambda}\left(R j_{*} \mathbb{C}_{S}\right)$ from any anti-dominant regular $\lambda_{0} \in \mathbb{R} \otimes_{\mathbb{Z}} \Lambda$ to $\lambda_{0}+\Lambda$, and therefore to all of $\mathbb{R} \otimes_{\mathbb{Z}} \Lambda$. In effect, this family is coherent in the strongest possible sense: defined for all $\lambda \in \mathbb{R} \otimes_{\mathbb{Z}} \Lambda$, with coefficients $d_{E, x}$ independent of $\lambda$. The constancy of the $d_{E, x}$ implies that the values of the function $\theta_{\lambda}\left(R j_{*} \mathbb{C}_{S}\right)$ on the regular set in $\mathfrak{g}_{\mathbb{R}}$ depend real analytically on $\lambda$. This makes the family of distributions $\theta_{\lambda}\left(R j_{*} \mathbb{C}_{S}\right)$ weakly analytic: the integral of the family against any test function is real analytic in the parameter $\lambda$.

There is a second family attached to the datum of the sheaf $\mathcal{F}=R j_{*} \mathbb{C}_{S}$, namely the right hand side of the equation in Theorem 3.8. Let us denote this family by $\tilde{\theta}_{\lambda}\left(R j_{*} \mathbb{C}_{S}\right)$. Proposition 7.15 asserts:

$$
\theta_{\lambda}\left(R j_{*} \mathbb{C}_{S}\right)=\tilde{\theta}_{\lambda}\left(R j_{*} \mathbb{C}_{S}\right) \text { when } \lambda \in \Lambda-\rho \text { is regular anti-dominant. }
$$

The definition of $\tilde{\theta}_{\lambda}\left(R j_{*} \mathbb{C}_{S}\right)$ involves integration over the characteristic cycle of the sheaf $R j_{*} \mathbb{C}_{S}$ and makes sense for every $\lambda \in \mathfrak{h}^{*}$, not just for $\lambda \in \Lambda-\rho$. The coadjoint orbit $\Omega(S, \lambda)$ as defined in (7.7) also has meaning for every $\lambda \in \mathbb{R} \otimes_{\mathbb{Z}} \Lambda$. Our proof of the identity (7.14) uses the fact that a positive integral multiple of $\lambda$ satisfies the positivity condition (7.3) - the integrality of $\lambda-\rho$ plays no role. Hence (7.14) remains valid for every anti-dominant regular $\lambda \in \mathbb{Q} \otimes_{\mathbb{Z}} \Lambda$. Combining this with (8.9), we conclude

$$
\theta_{\lambda}\left(R j_{*} \mathbb{C}_{S}\right)=\tilde{\theta}_{\lambda}\left(R j_{*} \mathbb{C}_{S}\right) \text { for } \lambda \in \mathbb{Q} \otimes_{\mathbb{Z}} \Lambda \text { regular anti-dominant. }
$$

Proposition 3.7 asserts that the family $\tilde{\theta}_{\lambda}\left(R j_{*} \mathbb{C}_{S}\right), \lambda \in \mathfrak{h}^{*}$, is weakly holomorphic in $\lambda$, in the sense that its value on any particular test function depends holomorphically on $\lambda$. In particular, it is weakly (real) analytic when restricted to $\mathbb{R} \otimes_{\mathbb{Z}} \Lambda$. Two weakly analytic families which coincide on a large enough set must coincide, hence, by (8.12):

8.13 Proposition. The character formula 3.8 is satisfied by every standard sheaf associated to an open $G_{\mathbb{R}}$-orbit, provided the group $G_{\mathbb{R}}$ contains a compact Cartan subgroup.

\section{INDUCTION}

In the previous two sections we proved our character formulas for standard sheaves associated to open orbits of groups $G_{\mathbb{R}}$ which contain a compact Cartan subgroup. We shall now extend the validity to standard sheaves attached to a larger class of orbits - roughly speaking orbits which fiber over a closed orbit in a generalized flag variety, with fibers of the type we have considered so far. In representation theoretic terms, we extend the validity of the character formulas by the process of parabolic induction. This has a counterpart on the level of standard sheaves, as we shall explain next.

Let $S=S\left(T_{\mathbb{R}}, \tau_{x_{0}}\right)=G_{\mathbb{R}} \cdot x_{0}$ be a $G_{\mathbb{R}^{-}}$orbit attached to a concrete Cartan subgroup $T_{\mathbb{R}} \subset G_{\mathbb{R}}$ and fixed point $x_{0}$ of $T_{\mathbb{R}}$, as in (6.5). The isomorphism $\tau_{x_{0}}: \mathfrak{t} \rightarrow$ $\mathfrak{h}$ determined by the fixed point $x_{0}$ pulls back the universal positive root system $\Phi^{+}$to a positive root system $\Phi_{S}^{+}(\mathfrak{g}, \mathfrak{t})$ in $\Phi(\mathfrak{g}, \mathfrak{t})$, the root system for $(\mathfrak{g}, \mathfrak{t})$. We call $\alpha \in \Phi(\mathfrak{g}, \mathfrak{t})$ real, imaginary, or complex depending on whether $\alpha=\bar{\alpha}(=$ complex 
conjugate of $\alpha), \alpha=-\bar{\alpha}$, or $\alpha \neq \pm \bar{\alpha}$. The following two properties of $\Phi_{S}^{+}(\mathfrak{g}, \mathfrak{t})$ are equivalent:

a) for every complex root $\alpha \in \Phi_{S}^{+}(\mathfrak{g}, \mathfrak{t})$, the root $\bar{\alpha}$ is also positive;

b) for every complex simple root $\alpha \in \Phi_{S}^{+}(\mathfrak{g}, \mathfrak{t})$, the root $\bar{\alpha}$ is positive.

Though phrased in terms of $T_{\mathbb{R}}$ and $x_{0}$, both a) and b) are really properties of the orbit $S$. The preceding statements are easy to verify - see [S4, lemma 6.14] for example. ${ }^{12}$ We shall call the orbit $S$ "maximally real" if it satisfies the equivalent conditions $(9.1 \mathrm{a}, \mathrm{b})$. It is these orbits that will be considered in the current section.

Let us suppose, then, that the orbit $S=S\left(T_{\mathbb{R}}, \tau_{x_{0}}\right)=G_{\mathbb{R}} \cdot x_{0}$ is maximally real. As explained in $\S 6$, standard sheaves attached to the orbit $S$ are determined by the following sets of data: a linear function $\lambda \in \mathfrak{h}^{*}$ and a character $\chi: T_{\mathbb{R}} \rightarrow \mathbb{C}^{*}$, such that $d \chi=\tau_{x_{0}}^{*}(\lambda-\rho)$; the pair $(\lambda, \chi)$ induces an irreducible, $G_{\mathbb{R}}$-equivariant, $(-\lambda-\rho)$-monodromic local system $\mathcal{L}_{\chi}$ on $S$. The direct image $\mathcal{F}=R j_{*} \mathcal{L}_{\chi}$ via the embedding $j: S \rightarrow X$ is the standard sheaf we shall work with.

We need to assemble various pieces of known structural information. To begin with, the Cartan subgroup $T_{\mathbb{R}}$ has a direct product decomposition

$$
T_{\mathbb{R}}=C_{\mathbb{R}} \cdot A_{\mathbb{R}}, \quad \text { with } C_{\mathbb{R}} \text { compact and } A_{\mathbb{R}} \text { connected and split. }
$$

The fact that $T_{\mathbb{R}}$ is fixed by an anti-holomorphic involution of the complex torus $T$ implies

$$
\begin{gathered}
C_{\mathbb{R}}=C_{\mathbb{R}}^{0} \cdot F \quad \text { (direct product), } \\
\text { with } F \subset G_{\mathbb{R}} \cap \exp \left(i \mathfrak{a}_{\mathbb{R}}\right), F \cong \mathbb{Z} / 2 \mathbb{Z} \times \cdots \times \mathbb{Z} / 2 \mathbb{Z} .
\end{gathered}
$$

In particular,

$$
\begin{aligned}
\chi & =\chi_{C} \cdot \chi_{A}, & & \text { with } \chi_{C}: C_{\mathbb{R}} \rightarrow \mathbb{C}^{*} \text { and } \chi_{A}: A_{\mathbb{R}} \rightarrow \mathbb{C}^{*}, \\
\chi_{C} & =\chi_{C}^{0} \cdot \chi_{F}, & & \text { with } \chi_{C}^{0}: C_{\mathbb{R}}^{0} \rightarrow \mathbb{C}^{*} \text { and } \chi_{F}: F \rightarrow \mathbb{C}^{*} .
\end{aligned}
$$

Since $C_{\mathbb{R}}^{0}$ and $A_{\mathbb{R}}$ are connected, the compatibility conditions

$$
d \chi_{C}^{0}=\left.\tau_{x_{0}}^{*}(\lambda-\rho)\right|_{\mathfrak{c}_{\mathbb{R}}}, \quad d \chi_{A}=\left.\tau_{x_{0}}^{*}(\lambda-\rho)\right|_{\mathfrak{a}_{\mathbb{R}}}
$$

completely determine $\chi_{C}^{0}$ and $\chi_{A}$. Following standard notation, we write the centralizer of $\mathfrak{a}$ as a direct product

$$
Z_{G}(\mathfrak{a})=M \cdot A \quad \text { (direct product), }
$$$$
\text { with } M \text { connected and defined over } \mathbb{R} \text {, }
$$

and correspondingly,

$$
\begin{aligned}
Z_{G_{\mathbb{R}}}(\mathfrak{a})= & M_{\mathbb{R}} \cdot F \cdot A_{\mathbb{R}} \quad \text { (direct product), } \\
& \text { where } M_{\mathbb{R}}=M \cap G_{\mathbb{R}} .
\end{aligned}
$$

We should warn the reader that $A_{\mathbb{R}}$ was defined to be the identity component of $A \cap G_{\mathbb{R}} ;$ in fact, $A \cap G_{\mathbb{R}}=A_{\mathbb{R}} \cdot F$. Because of our hypothesis (9.1)

$$
\mathfrak{v}=\bigoplus\left\{\mathfrak{g}^{-\alpha}\left|\alpha \in \Phi_{S}^{+}(\mathfrak{g}, \mathfrak{t}), \alpha\right|_{\mathfrak{a}} \neq 0\right\}
$$

\footnotetext{
12 [S4] treats the case of $K$-orbits, but also supplies the translation via Matsuki duality between $K$-orbits and $G_{\mathbb{R} \text {-orbits. }}$
} 
is a subalgebra of $\mathfrak{n}_{x_{0}}$, defined over $\mathbb{R}$, normalized by $M A$ but linearly disjoint from $\mathfrak{m} \oplus \mathfrak{a} ;$ it is the nilradical of the parabolic subalgebra

$$
\mathfrak{p}=\mathfrak{m} \oplus \mathfrak{a} \oplus \mathfrak{v} \quad \text { (semidirect product) } .
$$

Thus $P$, the normalizer of $\mathfrak{p}$ in $G$, is a parabolic subgroup, defined over $\mathbb{R}$, with Levi decomposition

$$
P=M \cdot A \cdot V \quad \text { (semidirect product). }
$$

Here $V \subset G$ denotes the connected subgroup with Lie algebra $\mathfrak{v}$; its group of real points $V_{\mathbb{R}}=V \cap G_{\mathbb{R}}$ is also connected. Unlike the complex parabolic $P$,

$$
P_{\mathbb{R}}={ }_{\text {def }} P \cap G_{\mathbb{R}}=M_{\mathbb{R}} \cdot A_{\mathbb{R}} \cdot F \cdot V_{\mathbb{R}}
$$

is not connected in general. In fact, the decomposition (9.7c) is topologically direct, so $F$ can be identified with the component group of $P_{\mathbb{R}}$.

The $G$-conjugates of $\mathfrak{p}$ constitute the underlying set of a generalized flag variety $Y$, the base of a $G$-equivariant fibration

$$
X \longrightarrow Y \quad \text { with fiber } X_{M} \cong \text { flag variety of } M \text {. }
$$

We identify $X_{M}$ concretely with the fiber through the point $x_{0}$. Then (9.8) induces a $G_{\mathbb{R}}$-equivariant fibration of orbits

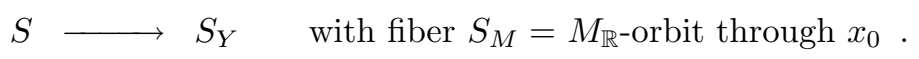

The $G_{\mathbb{R}}$-orbit $S_{Y} \subset Y$ can be identified with $G_{\mathbb{R}} / P_{\mathbb{R}}$, hence is a compact real form of

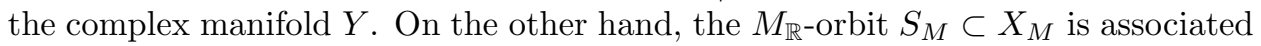
to the compact Cartan subgroup $C_{\mathbb{R}}^{0} \subset M_{\mathbb{R}}$, in the sense that $C_{\mathbb{R}}^{0}$ has $x_{0}$ as fixed point, hence is open. Since $M_{\mathbb{R}}$ stabilizes $X_{M}$,

$$
\mathcal{F}_{M}=\left.\mathcal{F}\right|_{X_{M}}
$$

is an object in $\mathrm{D}_{M_{\mathbb{R}}}\left(X_{M}\right)_{-\lambda_{M}}$, the twisted $M_{\mathbb{R}}$-equivariant derived category with twisting parameter $\lambda_{M}={ }_{\text {def }}$ restriction of $\lambda$ to the universal Cartan $\mathfrak{h}_{M}$ for $M$. Two comments are in order. We use the point $x_{0}$ to identify $\mathfrak{h} \cong \mathfrak{t}$ and $\mathfrak{h}_{M} \cong \mathfrak{c}$, and this allows us to regard $\mathfrak{h}_{m}$ as lying in $\mathfrak{h}$; the same choice of $x_{0}$ in the description (2.9) of the enhanced flag variety provides a distinguished embedding $\hat{X}_{M} \hookrightarrow \hat{X}$ compatible with the embedding $X_{M} \hookrightarrow X$ and the embedding of groups $M_{\mathbb{R}} \times H_{M} \hookrightarrow G_{\mathbb{R}} \times H$. The category $\mathrm{D}_{G_{\mathbb{R}}}(X)_{-\lambda}$ is built from $(-\lambda-\rho)$-monodromic sheaves, whereas the definition of $\mathrm{D}_{M_{\mathbb{R}}}\left(X_{M}\right)_{-\lambda_{M}}$ involves $\left(-\lambda_{M}-\rho_{M}\right)$-monodromic sheaves. Thus, to see that restriction from $X$ to $X_{M}$ maps $\mathrm{D}_{G_{\mathbb{R}}}(X)_{-\lambda}$ to $\mathrm{D}_{M_{\mathbb{R}}}\left(X_{M}\right)_{-\lambda_{M}}$, we need to know

$$
\rho_{M}=\text { restriction of } \rho \text { to } \mathfrak{h}_{M} \text { via } \mathfrak{h}_{M} \subset \mathfrak{h}
$$

this follows from our hypothesis (9.1). Tracing through the definitions, one finds:

9.12 Lemma. The restricted sheaf $\mathcal{F}_{M}$ is the standard sheaf associated to the open orbit $S_{M} \subset X_{M}$ and the data $\left(\lambda_{M}, \chi_{C}^{0}\right)$.

The character $\Theta(\mathcal{F})$ and its $M$-analogue $\Theta_{M}\left(\mathcal{F}_{M}\right)$ are related by parabolic induction. Recall the notion of normalized ${ }^{13}$ parabolic induction,

$$
\left(\tau, \chi_{F}, \nu\right) \mapsto I_{P_{\mathbb{R}}}^{G_{\mathbb{R}}}\left(\tau \otimes \chi_{F} \otimes e^{\nu}\right),
$$

\footnotetext{
${ }^{13}$ I.e., with built-in $\rho$-shift.
} 
which associates an admissible representation of $G_{\mathbb{R}}$, of finite length, to any triple $\left(\tau, \chi_{F}, \nu\right)$ consisting of an admissible $M_{\mathbb{R}}$-representation $\tau$ of finite length, a character $\chi_{F}: F \rightarrow \mathbb{C}^{*}$, and a linear function $\nu \in \mathfrak{a}^{*}$. The induction functor $I_{P_{\mathbb{R}}}^{G_{\mathbb{R}}}$ can also be applied to virtual representations, and hence to virtual characters.

9.14 Proposition. $\Theta(\mathcal{F})=I_{P_{\mathbb{R}}}^{G_{\mathbb{R}}}\left(\Theta_{M} \cdot \chi_{F} \cdot e^{\nu}\right)$, with $\Theta_{M}=\Theta_{M}\left(\mathcal{F}_{M}\right), \chi_{F}, \nu=\left.\lambda\right|_{\mathfrak{a}}$ as in (9.3), and $m=\operatorname{dim}_{\mathbb{C}} Y$.

In view of 9.12, when $\lambda_{M}$ is regular anti-dominant with respect to $M$, this is a statement about standard representations, which can be found in [SW]; alternatively - still in the anti-dominant situation - this follows from the duality theorem of [HMSW1]. Parabolic induction is compatible with coherent continuation - see, for example, [HS] - so the anti-dominance assumption can be dropped. Note that the standard sheaf $\mathcal{F}$ is the direct image of a locally constant sheaf on the orbit $S$, in degree zero; dually, $\mathbb{D} \mathcal{F}$ is concentrated in degree $-\left(m+2 \operatorname{dim}_{\mathbb{C}} X_{L}\right)$. On the other hand, the sheaf of hyperfunctions on $S_{Y}$ is the local cohomology sheaf along $Y$ of $\mathcal{O}_{Y}$, in degree $m$, so the sign changes one might expect cancel.

We should remark that the restriction operation $\mathrm{D}_{G_{\mathbb{R}}}(X)_{-\lambda} \rightarrow \mathrm{D}_{M_{\mathbb{R}}}\left(X_{M}\right)_{-\lambda_{M}}$ as in (9.10) factors,

$$
\mathrm{D}_{G_{\mathbb{R}}}(X)_{-\lambda} \rightarrow \mathrm{D}_{(M A)_{\mathbb{R}}}\left(X_{M A}\right)_{-\lambda} \rightarrow \mathrm{D}_{M_{\mathbb{R}}}\left(X_{M}\right)_{-\lambda_{M}} .
$$

The first of these arrows has a right adjoint, "sweeping out" along the orbit $S_{Y} \subset Y$, which is closed. On the level of representations, this corresponds to parabolic induction, whether or not the sheaf in question is a standard sheaf. We have chosen to go down all the way to $\mathrm{D}_{M_{\mathbb{R}}}\left(X_{M}\right)_{-\lambda_{M}}$ for technical reasons only: the group $M_{\mathbb{R}}$ has a compact Cartan subgroup, so that the discussion of $\S \S 7,8$ applies directly.

There exists an explicit formula for the induced character $I_{P_{\mathbb{R}}}^{G_{\mathbb{R}}}\left(\Theta_{M} \cdot \chi_{F} \cdot e^{\nu}\right)$, in terms of the inducing data $[\mathrm{Hi}]$, which we shall recall later. As one consequence of this formula, the restriction of the induced character to a small neighborhood of the identity depends only on $\Theta_{M}$ and $\nu$, not on $\chi_{F}$. Every connected component of $\mathfrak{g}_{\mathbb{R}}^{\prime}$, the set of regular semisimple elements in $\mathfrak{g}_{\mathbb{R}}$, meets any neighborhood of 0 . It follows that the pullback of the induced character to the Lie algebra does not depend on $\chi_{F}$. Accordingly, we suppress the symbol $\chi_{F}$ in the formula

$$
\theta(\mathcal{F})=I_{P_{\mathbb{R}}}^{G_{\mathbb{R}}}\left(\theta_{M} \cdot e^{\nu}\right) \quad\left(\theta_{M}=\theta_{M}\left(\mathcal{F}_{M}\right), \nu=\left.\lambda\right|_{\mathfrak{a}}\right),
$$

which is the Lie algebra analogue of 9.14 .

9.17 Proposition. The character formula 3.8 is satisfied by every standard sheaf associated to a maximally real orbit.

Proof. In effect, we must show that the character formula 3.8 is satisfied by the sheaf $\mathcal{F}=R j_{*} \mathcal{L}_{\chi}$ constructed at the beginning of this section, since every standard sheaf associated to a maximally real orbit is of this form. Just as in the group case, parabolic induction of characters on the level of the Lie algebra is given by an explicit formula. ${ }^{14}$ This formula can be applied to any invariant eigendistribution on $\mathfrak{m}_{\mathbb{R}}$, whether or not it comes from a character on the group $M_{\mathbb{R}}$. Moreover, when the induction process is applied to a holomorphic family of invariant eigendistributions on $\mathfrak{m}_{\mathbb{R}}$, the resulting invariant eigendistribution on $\mathfrak{g}_{\mathbb{R}}$ depends holomorphically on the parameter of the inducing family, and also on the parameter $\nu$. In $\S 8$ we

\footnotetext{
${ }^{14}$ Indeed, the formula on the Lie algebra can be deduced from that on the group.
} 
showed that $\theta_{M}=\theta_{M}\left(\mathcal{F}_{M}\right)$ - which is specified by the parameter $\left.\lambda\right|_{\mathfrak{c}}$ and an open

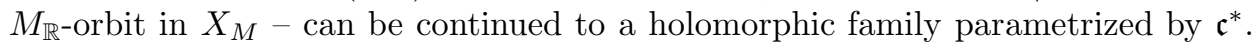
It follows that the induced character (9.16) depends holomorphically on $\lambda$. The characteristic cycle $\mathrm{CC}(\mathcal{F})$ depends only on the orbit $S$, so the family of invariant eigendistributions

$$
\phi \mapsto \frac{1}{(2 \pi i)^{n} n !} \int_{\mathrm{CC}(\mathcal{F})} \mu_{\lambda}^{*} \hat{\phi}\left(-\sigma+\pi^{*} \tau_{\lambda}\right)^{n} \quad\left(\lambda \in \mathfrak{h}^{*}\right)
$$

is holomorphic in the parameter $\lambda$ - cf. Proposition 3.7. Hence it suffices to prove the statement of the proposition under the following hypotheses:

$$
\text { a) } \lambda \text { is regular; } \quad \text { b) } \nu \in i \mathfrak{a}_{\mathbb{R}}^{*} \text {, }
$$

though we cannot assume that $\left.\lambda\right|_{\mathfrak{c}}$ is integral.

Let us describe the characteristic cycle $\operatorname{CC}(\mathcal{F})$ in terms of $\operatorname{CC}\left(\mathcal{F}_{M}\right)$. Locally near any point in $Y$, the fibration (9.8) is trivial; thus locally over $Y$ the sheaf $\mathcal{F}=R j_{*} \mathcal{L}_{\chi}$ is the exterior product of two sheaves: the direct images of the constant sheaves $\mathbb{C}_{S_{M}}$ and $\mathbb{C}_{S_{Y}}$ under, respectively, the open embedding $S_{M} \hookrightarrow X_{M}$ and the closed embedding $S_{Y} \hookrightarrow Y$. Correspondingly, $\operatorname{CC}(\mathcal{F})$ is - again locally over $Y$ the product of the characteristic cycle of the two sheaves. The orientation of the product cycle is independent of the order of the product, since both factors are evendimensional cycles. The orientation of the characteristic cycle of the direct image of $\mathbb{C}_{S_{M}}$ was pinned down in $\S 7$. We orient the conormal bundle $T_{S_{Y}}^{*} Y$ by means of the negative of the imaginary part of the holomorphic symplectic structure on $T^{*} Y$. We claim: $T_{S_{Y}}^{*} Y$, oriented as above, coincides with the characteristic cycle of the direct image of $\mathbb{C}_{S_{Y}}$. In effect, this is a statement about $\mathbb{R}^{m} \subset \mathbb{C}^{m}$; our conventions, as set up in [SV4], reduce this further to the case of $\mathbb{R} \subset \mathbb{C}$, where it can be checked directly.

We may as well suppose that the Cartan subgroup $T_{\mathbb{R}} \subset G_{\mathbb{R}}$ is invariant under the Cartan involution. The Levi component $M A$ of $P$ is then also invariant under the Cartan involution. Thus $M \cap U_{\mathbb{R}}$ - the intersection of $M$ with the compact real form $U_{\mathbb{R}} \subset G$ which was used to define the twisted moment map $\mu_{\lambda}$ - is a compact real form of $M$. We use it to define the twisted moment map

$$
\mu_{M, \lambda-\nu}: T^{*} X_{M} \longrightarrow \Omega_{M, \lambda-\nu}
$$

for $M$ and the twisting parameter $\left.\lambda\right|_{\mathfrak{c}}$, which we tacitly identify with $\lambda-\nu$; here $\Omega_{M, \lambda-\nu} \subset \mathfrak{m}^{*}$ denotes the $M$-orbit of $\left.\lambda\right|_{\mathfrak{c}}=\lambda-\nu$.

Next, we associate a cycle $C$ in $\Omega_{\lambda}$ to the cycle $\mu_{M, \lambda-\nu} \operatorname{CC}\left(\mathcal{F}_{M}\right)$ in $\Omega_{M, \lambda-\nu}$; the cycle $C$ will turn out to be the $\mu_{\lambda}$-image of $\operatorname{CC}(\mathcal{F})$. As a set,

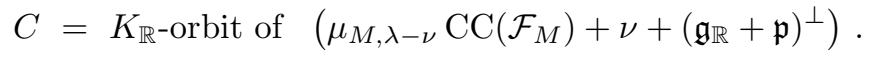

Here $\left(\mathfrak{g}_{\mathbb{R}}+\mathfrak{p}\right)^{\perp}$ refers to the annihilator in $\mathfrak{g}^{*}$ of $\left(\mathfrak{g}_{\mathbb{R}}+\mathfrak{p}\right)^{\perp}$, relative to the real part of the pairing $\mathfrak{g} \times \mathfrak{g}^{*} \rightarrow \mathbb{C}$; under the isomorphism $\mathfrak{g}^{*} \cong \mathfrak{g}$ induced by the Killing form, $\left(\mathfrak{g}_{\mathbb{R}}+\mathfrak{p}\right)^{\perp}$ corresponds to $i \mathfrak{v}_{\mathbb{R}}$. Locally near $\lambda, C$ splits into a product $C \cong \mu_{M, \lambda-\nu} \mathrm{CC}\left(\mathcal{F}_{M}\right) \times C^{\prime}$, where $C^{\prime}$ is a submanifold of $\mathfrak{m}^{\perp} \cap i \mathfrak{g}_{\mathbb{R}}^{*}$, passing through $\nu$ (recall $(9.19 \mathrm{~b}) !)$, with tangent space $(\mathfrak{m}+\mathfrak{a})^{\perp} \cap i \mathfrak{g}_{\mathbb{R}}^{*}$ at $\nu$. Note that $(\mathfrak{m}+\mathfrak{a})^{\perp} \cap i \mathfrak{g}_{\mathbb{R}}^{*}$ corresponds to $i \mathfrak{v}_{\mathbb{R}} \oplus i\left(\mathfrak{v}_{\text {opp }}\right)_{\mathbb{R}}$. Once this tangent space is oriented, its $K_{\mathbb{R}}$-translates become consistently oriented, providing an orientation of $C^{\prime}$. Finally, we orient

$$
T_{\nu} C^{\prime} \cong(\mathfrak{m}+\mathfrak{a})^{\perp} \cap i \mathfrak{g}_{\mathbb{R}}^{*}
$$


by means of the imaginary part of the holomorphic symplectic form of $\Omega_{\lambda}$. This form is nondegenerate on the image of $(\mathfrak{m}+\mathfrak{a})^{\perp}$ in $T_{\lambda} \Omega_{\lambda}$, and defined over $\mathbb{R}$ relative to the natural real structure of $(\mathfrak{m}+\mathfrak{a})^{\perp}$; therefore its imaginary part does specify an orientation of the space (9.22). Since $\mu_{M, \lambda-\nu} \operatorname{CC}\left(\mathcal{F}_{M}\right)$ is a cycle, the orientation of $C^{\prime}$ now determines $C$ as a cycle.

9.23 Lemma. $\mu_{\lambda} \operatorname{CC}(\mathcal{F})=C$.

We shall prove the lemma after completing the proof of Proposition 9.17. Because of the hypothesis (9.19a), we can rewrite the integral (9.18) and its $M$-analogue as integrals over the cycles $\mu_{\lambda}(\mathrm{CC}(\mathcal{F})) \subset \Omega_{\lambda}$, respectively $\mu_{M, \lambda-\nu}\left(\mathrm{CC}\left(\mathcal{F}_{M}\right)\right) \subset$ $\Omega_{M, \lambda-\nu}$, as in (3.9). The integral over $\mu_{M, \lambda-\nu}\left(\operatorname{CC}\left(\mathcal{F}_{M}\right)\right)$ represents the virtual character $\theta_{M}\left(\mathcal{F}_{M}\right)$ - that is, the main result of the previous section. We need to show that the integral over the cycle $\mu_{\lambda}(\mathrm{CC}(\mathcal{F}))$ represents the virtual character (9.16). Lemma 9.23 reduces the problem to the following assertion: if a virtual character $\theta_{M}$ on the Lie algebra $\mathfrak{m}_{\mathbb{R}}$ is represented as an integral of the type (3.9) over the cycle $C_{M}$ in $\Omega_{M, \lambda-\nu}$, then the induced character $I_{P_{\mathbb{R}}}^{G_{\mathbb{R}}}\left(\theta_{M} \cdot e^{\nu}\right)$ is represented by the integral over the cycle $C$ defined below (9.21), with the unspecified cycle $C_{M}$ playing the role of $\mu_{M, \lambda-\nu}\left(\mathrm{CC}\left(\mathcal{F}_{M}\right)\right)$. This last assertion is established by Rossmann [R2, §2], who generalizes an argument of Duflo [D1]. Rossmann specifies the orientation of the "induced cycle" in $\Omega_{\lambda}$ only implicitly; a careful examination of his proof shows that he uses the symplectic form of $\Omega_{\lambda}$, exactly as we have done in the description of $C$. At this point the proof of Proposition 9.17 is complete, except for the verification of 9.23 .

Proof of Lemma 9.23. The two cycles are $K_{\mathbb{R}}$-invariant, as is the moment map $\mu_{\lambda}$, and $K_{\mathbb{R}}$ acts transitively on $S_{Y}$. It therefore suffices to identify the two cycles over a single point in $S_{Y}$. The embedding $(\mathfrak{m} \oplus \mathfrak{a})^{*} \hookrightarrow \mathfrak{g}^{*}$ induced by the Killing form which was used to identify $X_{M}$ with a specific fiber of $X \rightarrow Y$ can also be used to embed $T^{*} X_{M} \hookrightarrow T^{*} X$ compatibly with $X_{M} \hookrightarrow X$ and $M A$-equivariantly. The twisted moment maps $\mu_{\lambda}$ and $\mu_{M, \lambda-\nu}$ fit into a commutative diagram

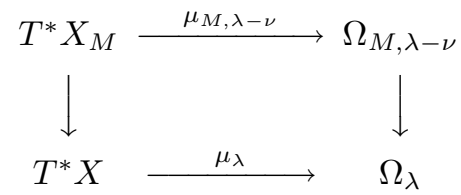

in which the right vertical arrow is induced by $\mathfrak{m}^{*} \hookrightarrow \mathfrak{g}^{*}$, followed by translation by $\nu$. Over the base point $y_{0}$ in $S_{Y}$, our passage from $\operatorname{CC}\left(\mathcal{F}_{M}\right)$ to $\operatorname{CC}(\mathcal{F})$ as explained above amounts to adding the inverse image in $T^{*} X$ of the conormal space $\left(T_{S_{Y}}^{*} Y\right)_{y}$ to $\operatorname{CC}\left(\mathcal{F}_{M}\right)$ and taking the $K_{\mathbb{R}}$-saturation. Via the twisted moment map $\mu_{\lambda}$, the inverse image of $\left(T_{S_{Y}}^{*} Y\right)_{y_{0}}$ maps isomorphically to the $\nu$-translate of $\left(\mathfrak{g}_{\mathbb{R}}+\mathfrak{p}\right)^{\perp}$, so the passage from $\mu_{M, \lambda-\nu}\left(\mathrm{CC}\left(\mathcal{F}_{M}\right)\right)$ to $C$ is the isomorphic $\mu_{\lambda}$-image of the passage from $\operatorname{CC}\left(\mathcal{F}_{M}\right)$ to $\operatorname{CC}(\mathcal{F})$, except possibly for the orientation. We used the symplectic form of $\Omega_{\lambda}$ to orient $\left(\mathfrak{g}_{\mathbb{R}}+\mathfrak{m}+\mathfrak{a}\right)^{\perp}$ and the symplectic form of $T^{*} Y$ to orient $T_{S_{Y}}^{*} Y$. We also regarded $X$, locally over $y_{0}$, as isomorphic to the product $X_{M} \times Y$; this allows us to regard $T^{*} Y$ as a factor of $T^{*} X$ and to restrict the symplectic form of $T^{*} X$ to $T^{*} Y$, where it must agree with its symplectic form. In other words, the symplectic form of $T^{*} X$ can be used to orient $T_{S_{Y}}^{*} Y$. Proposition 3.3 relates the symplectic form of $\Omega_{\lambda}$ to that of $T^{*} X$ : they correspond to each other via $\mu_{\lambda}$, except for a sign change and the addition of the two-form $\pi^{*} \tau_{\lambda}$. Since 
that form is pulled back from the base, it can be checked that $\left(-\sigma+\pi^{*} \tau_{\lambda}\right)^{m}$ agrees with $(-\sigma)^{m}$ on $T_{S_{Y}}^{*} Y$ - what matters here is that the (real) $2 m$-manifold $T_{S_{Y}}^{*} Y$ is the conormal bundle of a (real) $m$-manifold. Since we had oriented $T_{S_{Y}}^{*} Y$ by the imaginary part of $-\sigma$, we conclude that the two cycles $\mu_{\lambda} \operatorname{CC}(\mathcal{F})$ and $C$ agree, as asserted by the lemma.

In effect, we have shown that the integral formula 3.8 is compatible with parabolic induction. We need to do the same for the fixed point formula:

9.25 Proposition. The character formulas 5.12, 5.24, and 5.27 are satisfied by every standard sheaf associated to a maximally real orbit.

Proof. Because of Proposition 6.2, we only need to show that one of the two formulas in Theorem 5.24 for the $c_{t, x}$ is compatible with parabolic induction - that not only implies the compatibility with induction of the formula 5.12, but also of 5.27 , which is really a special case of 5.24 .

We begin by recalling the explicit formula for the character of an induced representation in terms of the inducing character [Hi]. Let $\Theta_{M}, \chi_{F}$, and $\nu \in \mathfrak{a}^{*}$ be inducing data as in 9.14. Then $I_{P_{\mathbb{R}}}^{G_{\mathbb{R}}}\left(\Theta_{M} \cdot \chi_{F} \cdot e^{\nu}\right)$ is supported on the union of the $G_{\mathbb{R}^{-}}$conjugates of $Z_{G_{\mathbb{R}}}(\mathfrak{a})=M_{\mathbb{R}} \cdot F \cdot A_{\mathbb{R}}$, so it suffices to describe this induced character on any Cartan subgroup of $G_{\mathbb{R}}$ which is contained in $M_{\mathbb{R}} \cdot F \cdot A_{\mathbb{R}}$. Since we have used the symbol $T_{\mathbb{R}}$ for the specific Cartan subgroup used in describing the orbit $S$, let us write $\tilde{T}_{\mathbb{R}}$ for a typical Cartan subgroup contained in $M_{\mathbb{R}} \cdot F \cdot A_{\mathbb{R}}$. Then, for any regular $t \in \tilde{T}_{\mathbb{R}}$,

$$
\begin{aligned}
& \left(I_{P_{\mathbb{R}}}^{G_{\mathbb{R}}}\left(\Theta_{M} \cdot \chi_{F} \cdot e^{\nu}\right) \prod_{\alpha \in \Phi^{+}}\left|e^{\alpha / 2}-e^{-\alpha / 2}\right|\right)(t) \\
& \quad=\sum_{g \in R / \tilde{T}_{\mathbb{R}}}\left(\left(\Theta_{M} \cdot \chi_{F} \cdot e^{\nu}\right) \prod_{\alpha \in \Phi_{M}^{+}}\left|e^{\alpha / 2}-e^{-\alpha / 2}\right|\right)\left(g t g^{-1}\right),
\end{aligned}
$$

where $R=\left\{g \in G_{\mathbb{R}} \mid g \tilde{T}_{\mathbb{R}} g^{-1} \subset M A\right\}$.

In this formula, the particular choices of positive root system $\Phi^{+}$for the pair $(\mathfrak{g}, \tilde{\mathfrak{t}})$ and $\Phi_{M}^{+}$for $(\mathfrak{m} \oplus \mathfrak{a}, \tilde{\mathfrak{t}})$ do not matter since we are taking absolute values. On the right in (9.26), and in various formulas below, we evaluate the functions $\Theta_{M}, \chi_{F}$, and $e^{\nu}$ at points $g t g^{-1}$, with $g \in R$ and $t \in \tilde{T}_{\mathbb{R}}$, in which case $g t g^{-1}$ lies in the direct product $M_{\mathbb{R}} \cdot F \cdot A_{\mathbb{R}}$; thus $\Theta_{M}\left(g t g^{-1}\right)$ denotes the value of $\Theta_{M}$ at the $M_{\mathbb{R}}$-component of $g t g^{-1}$, and with the analogous convention in the other two cases.

Let us re-write both sides of (9.26) in terms of the constants $c_{t, x}$ as in (4.3). With $t \in \tilde{T}_{\mathbb{R}}$ regular and $\zeta \in \tilde{\mathfrak{t}}_{\mathbb{R}}$ sufficiently small,

$$
I_{P_{\mathbb{R}}}^{G_{\mathbb{R}}}\left(\Theta_{M} \cdot \chi_{F} \cdot e^{\nu}\right)(t \exp (\zeta))=\sum_{x \in X^{\tilde{T}_{\mathbb{R}}}} \frac{c_{t, x} e^{\lambda_{x}(\zeta)-\rho_{x}(\zeta)}}{\prod_{\alpha \in \Phi^{+}}\left(1-e^{-\alpha_{x}}\right)(t \exp (\zeta))} .
$$

On the other hand,

$$
\Theta_{M}(t \exp (\zeta))=\sum_{x \in X_{M}^{\widetilde{T}_{R}}} \frac{c_{t, x}^{M} e^{(\lambda-\nu)_{x}(\zeta)-\rho_{M, x}(\zeta)}}{\prod_{\alpha \in \Phi_{M}^{+}}\left(1-e^{-\alpha_{x}}\right)(t \exp (\zeta))},
$$


hence

$$
\begin{aligned}
& \left(\Theta_{M} \cdot \chi_{F} \cdot e^{\nu}\right)\left(g t \exp (\zeta) g^{-1}\right) \\
& =\sum_{x \in X_{M}^{g \tilde{T}_{\mathbb{R}}-1}} \frac{e^{\nu}\left(g t g^{-1}\right) \chi_{F}\left(g t g^{-1}\right) c_{g t g^{-1}, x}^{M} e^{\lambda_{x}\left(g \zeta g^{-1}\right)-\rho_{M, x}\left(g \zeta g^{-1}\right)}}{\prod_{\alpha \in \Phi_{M}^{+}}\left(1-e^{-\alpha_{x}}\right)\left(g t g^{-1} \exp \left(g \zeta g^{-1}\right)\right)} .
\end{aligned}
$$

Unlike in (9.26), $\Phi^{+}$and $\Phi_{M}^{+}$in (9.27)-(9.29) refer to the universal positive root systems. The notation $c_{g t g^{-1}, g x}^{M}$ is slightly misleading, since $g t g^{-1}$ may lie in $M_{\mathbb{R}}$. $A_{\mathbb{R}} \cdot F$ rather than $M_{\mathbb{R}}$ so we need to take the $M_{\mathbb{R}}$-component of $g t g^{-1}$. Note that $\chi_{F}$ is constant on connected components, which explains why we can omit the factor $\chi_{F}\left(g \zeta g^{-1}\right)$. We should remark that $\nu$ is used in two different but compatible senses. On the one hand, as a linear function on $\mathfrak{a}$, on the other as a linear function on the universal Cartan $\mathfrak{h}$, identified with the universal Cartan of $\mathfrak{m} \oplus \mathfrak{a}$, so that $\mathfrak{a} \subset \mathfrak{h}$. When we use a fixed point $x$ of $\tilde{T}_{\mathbb{R}}$ in $X_{M}$ to identify $\mathfrak{h} \cong \tilde{\mathfrak{t}}$, the restriction of $\lambda_{x}$ to $\mathfrak{a} \subset \tilde{\mathfrak{t}}$ agrees with $\nu=\nu_{x}$.

As $g$ ranges over $R / \tilde{T}_{\mathbb{R}}$ and $x$ over the fixed points of $g \tilde{T}_{\mathbb{R}} g^{-1}$ in $X_{M}$, the translates $g^{-1} x$ range over the fixed points of $\tilde{T}_{\mathbb{R}}$ in $X$ which lie in $p^{-1} S_{Y}$, the inverse image of the closed orbit $S_{Y} \subset Y$. Given such a fixed point $z=g^{-1} x$, the original fixed point $x$ and $g \in G_{\mathbb{R}}$ are determined by $z$ up to left translation, respectively left multiplication, by an element of $G_{\mathbb{R}} \cap M A=M_{\mathbb{R}} \cdot F \cdot A_{\mathbb{R}}$. Note also that $\lambda_{g x}\left(g \zeta g^{-1}\right)=\lambda_{x}(\zeta)$, with similar identities for $\rho_{M, x}, \alpha_{x}$, etc. Thus, substituting (9.29) into the quantity on the right in (9.26), we find

$$
\begin{aligned}
& \left(I_{P_{\mathbb{R}}}^{G_{\mathbb{R}}}\left(\Theta_{M} \cdot \chi_{F} \cdot e^{\nu}\right) \prod_{\alpha \in \Phi^{+}}\left|e^{\alpha / 2}-e^{-\alpha / 2}\right|\right)(t \exp (\zeta)) \\
& =\sum_{x \in X^{\tilde{T}_{\mathbb{R}} \cap p^{-1} S_{Y}}} e^{\nu}\left(g t g^{-1}\right) \chi_{F}\left(g t g^{-1}\right) c_{g t g^{-1}, g x}^{M} e^{\lambda_{x}(\zeta)-\rho_{M, x}(\zeta)} \\
& \quad \times\left|e^{\rho_{M, x}}(t \exp (\zeta))\right| \prod_{\alpha \in \Phi_{M}^{+}} \frac{\left|1-e^{-\alpha_{x}}\right|}{\left(1-e^{-\alpha_{x}}\right)}(t \exp (\zeta)) ;
\end{aligned}
$$

in this formula, the $g=g(x) \in G_{\mathbb{R}}$ are chosen so that $g^{-1} x \in X_{M}$, in which case it is unique up to left multiplication by some $m \in M_{\mathbb{R}} \cdot F \cdot A_{\mathbb{R}}$ - that makes the various terms involving $g$ well defined. We continue by substituting (9.27) into the left hand side of (9.30),

$$
\begin{aligned}
& \sum_{x \in X^{\tilde{T}_{\mathbb{R}}}} c_{t, x} e^{\lambda_{x}(\zeta)-\rho_{x}(\zeta)}\left|e^{\rho_{x}}(t \exp (\zeta))\right| \prod_{\alpha \in \Phi^{+}} \frac{\left|1-e^{-\alpha_{x}}\right|}{\left(1-e^{-\alpha_{x}}\right)}(t \exp (\zeta)) \\
& =\sum_{x \in X^{\tilde{T}_{\mathbb{R}} \cap p^{-1} S_{Y}}} e^{\nu}\left(g t g^{-1}\right) \chi_{F}\left(g t g^{-1}\right) c_{g t g^{-1}, g x}^{M} e^{\lambda_{x}(\zeta)-\rho_{M, x}(\zeta)} \\
& \quad \times\left|e^{\rho_{M, x}}(t \exp (\zeta))\right| \prod_{\alpha \in \Phi_{M}^{+}} \frac{\left|1-e^{-\alpha_{x}}\right|}{\left(1-e^{-\alpha_{x}}\right)}(t \exp (\zeta)) .
\end{aligned}
$$

Comparing the two sides in (9.31), we obtain the following re-statement of the induced character formula (9.26) in terms of the $c_{t, x}$. First,

$$
c_{t, x}=0 \quad \text { unless } x \in p^{-1} S_{Y}
$$


in words, the induced character is supported on the union of the conjugates of the inducing subgroup $P_{\mathbb{R}}$. Secondly,

$$
\begin{gathered}
c_{t, x}=(-1)^{k_{x}} e^{\nu}\left(g t g^{-1}\right) \frac{\chi_{F}\left(g t g^{-1}\right)}{\left|e^{\left(\rho_{x}-\rho_{M, x}\right)}(t)\right|} c_{g t g^{-1}, g x}^{M} \text { if } x \in X^{\tilde{T}_{\mathbb{R}}} \cap p^{-1} S_{Y}, \\
\text { with } k_{x}=\#\left\{\alpha \in \Phi^{+}-\Phi_{M}^{+} \mid 0<e^{\alpha_{x}}(t)<1\right\} \text { and } \quad g^{-1} x \in X_{M} .
\end{gathered}
$$

We should explain that nonreal roots occur in pairs, so the quotient of the products on the two sides of (9.31) is \pm 1 , with the sign governed by the number of positive real roots such that $e^{-\alpha_{x}}(t)>1$, i.e., by the number $k_{x}$. Also, $\left|e^{\left(\rho_{x}-\rho_{M, x}\right)}(\exp \zeta)\right|=$ $e^{\left(\rho_{x}-\rho_{M, x}\right)}(\exp \zeta)$ since $\rho_{x}-\rho_{M, x}$ is real valued.

To complete the proof, we must show that the relation (9.32) between the $c_{t, x}$ and $c_{t, x}^{M}$ is consistent with one of the two formulas in (9.24) when we express $c_{t, x}$ in terms of $\mathcal{F}$ and $c_{t, x}^{M}$ in terms of $\mathcal{F}_{M}$. The sheaf $\mathcal{F}$ is supported on $p^{-1}\left(S_{Y}\right)$ so any quantity attached to $\mathcal{F}$ by a local construction - such as either of the two Lefschetz numbers in (5.24) - vanish at points not in $p^{-1}\left(S_{Y}\right)$. Thus we only need to check the consistency of $(9.32 \mathrm{~b})$. Let us reduce the problem to the case when the fixed point $x$ lies in $X_{M}$, so that we can choose $g=e$; the legitimacy of this reduction reflects the fact that the choice of the base point $y_{0}$ over which the reference fiber $X_{M}$ lies is arbitrary.

After the reduction, we are dealing with a point $x \in X_{M}$ fixed by the Cartan subgroup $\tilde{T}_{\mathbb{R}} \subset M_{\mathbb{R}} A_{\mathbb{R}} F$ and a regular element $t \in \tilde{T}_{\mathbb{R}}$. The open Schubert cell $N^{+}(t, x)$ defined in (5.17) splits naturally into a product

$$
\begin{aligned}
& V^{+} \times N_{M}^{+}(t, x) \stackrel{\sim}{\rightarrow} N^{+}(t, x), \quad(v, \tilde{x}) \mapsto v \cdot \tilde{x}, \\
& \text { with } \quad V^{+}=\exp \left(\oplus_{\alpha \in \Phi^{+-} \Phi_{M}^{+}} \mathfrak{g}^{\alpha_{x}}\right), \quad N_{M}^{+}(t, x)=N^{+}(t, x) \cap X_{M} .
\end{aligned}
$$

The group $V^{+}$is the opposite to the unipotent radical $V$ of $P$; its orbit $V^{+} \cdot y_{0}$ is the open Schubert cell in $Y$ around $y_{0}$. Note that $V_{\mathbb{R}}^{+} \cdot y_{0}$, the open Schubert cell in $S_{Y} \cong G_{\mathbb{R}} / P_{\mathbb{R}}$, coincides with the intersection of the complex Schubert cell $V^{+} \cdot y_{0}$ with the $G_{\mathbb{R}}$-orbit $S_{Y}$. Thus the $S \cap N^{+}(t, x)$ splits into a product

$$
S \cap N^{+}(t, x) \cong V_{\mathbb{R}}^{+} \times\left(S_{M} \cap N_{M}^{+}(t, x)\right),
$$

compatibly with (9.33). The choice of the base point $y_{0}$ allows us to regard $A$ as a subgroup of the universal Cartan $H$, in fact, $H \cong A \times H_{M}$. This splitting, too, is compatible with (9.33), as is the splitting

$$
N^{+}(t, x) \times H \cong\left(V^{+} \times A\right) \times\left(N_{M}^{+}(t, x) \times H_{M}\right)
$$

of $N^{+}(t, x) \times H$, the inverse image of $N^{+}(t, x)$ in the enhanced flag variety $\hat{X}$. The monodromy data defining the sheaf $\mathcal{F}$ split into a product corresponding to the splitting (9.35), of the two characters

$$
\begin{aligned}
\chi_{A} \cdot \chi_{F} & : A \cap G_{\mathbb{R}} \longrightarrow \mathbb{C}^{*}, \\
\chi_{C}^{0} & : C_{\mathbb{R}}^{0} \longrightarrow \mathbb{C}^{*} ;
\end{aligned}
$$

recall that $A \cap G_{\mathbb{R}}=A_{\mathbb{R}} \cdot F$. The monodromic sheaf $\mathcal{F}$, regarded as a sheaf on $N^{+}(t, x) \times H$, decomposes into an exterior product of monodromic sheaves on the two factors, with monodromy data (9.36). According to 9.12, one of these is the $\left(-\lambda_{M}-\rho_{M}\right)$-monodromic sheaf $\mathcal{F}_{M}$, which corresponds to the character $\chi_{C}^{0}$, though now we think of it as a sheaf on $N_{M}^{+}(t, x) \times H_{M}$. The other is an $A \cap G_{\mathbb{R}}$-equivariant, 
$A$-monodromic sheaf $\mathcal{F}_{V}$ on $V^{+}$, regarded as a sheaf on $V^{+} \times A$. This sheaf $\mathcal{F}_{V}$ is

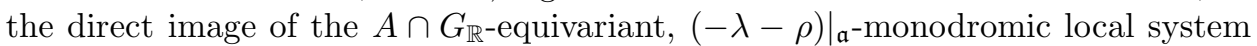
$\mathcal{L}_{\chi, V}$ on $V_{\mathbb{R}}^{+}$associated to the character $\left.\chi\right|_{A \cap G_{\mathbb{R}}}=\chi_{A} \cdot \chi_{F}$. Note that

$$
d\left(\chi_{A} \cdot \chi_{F}\right)=d\left(\left.\chi\right|_{A \cap G_{\mathbb{R}}}\right)=\left.(d \chi)\right|_{\mathfrak{a}}=\nu-\left(\rho_{x}-\rho_{M, x}\right),
$$

provided $x \in X_{M}$ - recall (9.11) and the definition of $\nu$ in 9.14 .

Kashiwara's fixed point formalism is functorial with respect to exterior products of sheaves. Let us argue that the subspaces $N^{\prime}(t, x), N^{\prime \prime}(t, x)$ of $N^{+}(t, x)$ defined in (5.17) split compatibly with the product decompositions (9.33)-(9.34); what makes this true is the fact that both are orbits through $x$ of subgroups of $\exp \left(\mathfrak{n}^{+}(t, x)\right)$ whose Lie algebras are sums of root spaces - any such group is the semidirect product of its intersections with $V^{+}$and $\exp \left(\mathfrak{n}_{M}^{+}(t, x)\right)$. Recall the passage $\mathcal{F} \rightsquigarrow$ $\mathcal{F}(x)$ described in (5.19), with now $\tilde{T}_{\mathbb{R}}$ taking the place of $T_{\mathbb{R}}$. We use the analogous notation for $\mathcal{F}_{M}$ and $\mathcal{F}_{V}$. The Künneth isomorphism

$$
\mathcal{H}_{N^{\prime}(t, x)}^{*}(\mathbb{D} \mathcal{F}(x))_{x} \cong \mathcal{H}_{V^{+} \cap \exp \mathfrak{n}^{\prime}(t, x)}^{*}\left(\mathbb{D} \mathcal{F}_{V}(e)\right)_{e} \otimes \mathcal{H}_{N_{M}^{\prime}(t, x)}^{*}\left(\mathbb{D} \mathcal{F}_{M}(x)\right)_{x}
$$

gives us the first Lefschetz number in Theorem 5.24 as the product of the two Lefschetz numbers

$$
\begin{gathered}
\sum(-1)^{i} \operatorname{tr}\left(\phi_{t}: \mathcal{H}_{V^{\prime}}^{i}\left(\mathbb{D} \mathcal{F}_{V}(e)\right)_{e} \rightarrow \mathcal{H}_{V^{\prime}}^{i}\left(\mathbb{D} \mathcal{F}_{V}(e)\right)_{e} \otimes \mathbb{C}_{\nu}\right), \\
\sum(-1)^{i} \operatorname{tr}\left(\phi_{t}: \mathcal{H}_{N_{M}^{\prime}(t, x)}^{i}\left(\mathbb{D} \mathcal{F}_{M}(x)\right)_{x} \rightarrow \mathcal{H}_{N_{M}^{\prime}(t, x)}^{i}\left(\mathbb{D} \mathcal{F}_{M}(x)\right)_{x} \otimes \mathbb{C}_{\lambda_{M}}\right),
\end{gathered}
$$

with $V^{\prime}=V^{+} \cap \exp \mathfrak{n}^{\prime}(t, x)$. Here $\mathbb{C}_{\nu}$ is the local system on $A$ with generating section $e^{\nu-\rho+\rho_{M}}-$ recall (9.11) and the notation $\nu=\left.\lambda\right|_{\mathfrak{a}}-$ and $\mathbb{C}_{\lambda_{M}}$ the local system on $H_{M}$ with generating section $e^{\lambda_{M}-\rho_{M}}$. Their exterior product, we remark, is the local system $\mathbb{C}_{\lambda}$ on $H$.

In the previous section we proved the special instance of Theorem 5.24 involving the group $M_{\mathbb{R}}$ and the sheaf $\mathcal{F}_{M}$. In effect, this identifies the second of the two Lefschetz numbers as the constant $c_{t, x}^{M}$ in $(9.32 \mathrm{~b})$. The constant $c_{t, x}$ on the left in (9.32b) corresponds to the induced character (9.27); because of Proposition 9.14, $c_{t, x}$ is the constant that corresponds to $\Theta(\mathcal{F})$. Thus, to complete the proof, we must show:

$$
\begin{gathered}
(-1)^{k_{x}} e^{\nu}(t) \frac{\chi_{F}(t)}{\left|e^{\left(\rho_{x}-\rho_{M, x}\right)}(t)\right|} \\
=\sum(-1)^{i} \operatorname{tr}\left(\phi_{t}: \mathcal{H}_{V^{\prime}}^{i}\left(\mathbb{D} \mathcal{F}_{V}(e)\right)_{e} \rightarrow \mathcal{H}_{V^{\prime}}^{i}\left(\mathbb{D} \mathcal{F}_{V}(e)\right)_{e} \otimes \mathbb{C}_{\nu}\right) ;
\end{gathered}
$$

as was pointed out, we are free to assume $g=e$ in (9.32b), so $g$ no longer appears in (9.40). The first of the two Lefschetz numbers in (9.39) only depends on the component of $t$ in $A \cap G_{\mathbb{R}}$, so we assume $t \in A \cap G_{\mathbb{R}}$ from now on.

The standard sheaf $\mathcal{F}$ was constructed as the direct image of the twisted local system $\mathcal{L}_{\chi}$ on the $G_{\mathbb{R}^{-}}$orbit $S \subset X$. As was remarked earlier, $\mathcal{F}_{V}$ is the direct image of the local system $\mathcal{L}_{\chi, V}$ under the closed embedding $V_{\mathbb{R}}^{+} \hookrightarrow V^{+}$. The operations of Verdier duality and local cohomology along a closed subspace commute with direct image under a closed embedding. This allows us to reduce (9.40) to a statement about the action of $t$ on the space $V_{\mathbb{R}}^{+}$and the twisted local system $\mathcal{L}_{\chi, V}$ :

$$
\begin{gathered}
(-1)^{k_{x}} e^{\nu}(t) \frac{\chi_{F}(t)}{\left|e^{\left(\rho_{x}-\rho_{M, x}\right)}(t)\right|} \\
=\sum(-1)^{i} \operatorname{tr}\left(\phi_{t}: \mathcal{H}_{V_{\mathbb{R}}^{\prime}}^{i}\left(\mathbb{D} \mathcal{L}_{\chi, V}\right)_{e} \rightarrow \mathcal{H}_{V_{\mathbb{R}}^{\prime}}^{i}\left(\mathbb{D} \mathcal{L}_{\chi, V}\right)_{e} \otimes \mathbb{C}_{\nu}\right) ;
\end{gathered}
$$


here $V_{\mathbb{R}}^{\prime}$ denotes the intersection $V^{\prime} \cap V_{\mathbb{R}}^{+}=V_{\mathbb{R}}^{+} \cap \mathfrak{n}^{\prime}(t, x)$ and $\phi_{t}$ the morphism induced by the $\phi$ corresponding to $\mathbb{D} \mathcal{L}_{\chi, V}$. Let $\mathcal{L}_{\chi, V}^{*}=\mathcal{H}$ om $\left(\mathcal{L}_{\chi, V}, \mathbb{C}_{V_{\mathbb{R}}^{+}}\right)$denote the local system dual to $\mathcal{L}_{\chi, V}$. Then

$$
\mathbb{D} \mathcal{L}_{\chi, V}=\mathcal{L}_{\chi, V}^{*} \otimes \mathbb{D}_{V_{\mathbb{R}}^{+}},
$$

as equivariant twisted sheaves. Correspondingly, the quantity on the right of (9.41) becomes a product of two numbers. First, the action of $t$ via $\phi$ on the stalk of $\mathcal{L}_{\chi, V}^{*}$ at the fixed point $e$ : the structure of an $\left(A \cap G_{\mathbb{R}}\right)$-equivariant, $\left.(-\lambda-\rho)\right|_{\mathfrak{a}}$-monodromic sheaf on $\mathcal{L}_{\chi, V}^{*}$ gives us a morphism $\phi: t^{*} \mathcal{L}_{\chi, V}^{*} \rightarrow \mathcal{L}_{\chi, V}^{*} \otimes \mathbb{C}_{\nu}$, in complete analogy to (5.22); here, as in $\S 5$, we think of $t$ as variable in $A \cap G_{\mathbb{R}}$. At the fixed point $e$, $\phi$ induces a morphism of stalks $\phi:\left(\mathcal{L}_{\chi, V}^{*}\right)_{e} \rightarrow\left(\mathcal{L}_{\chi, V}^{*}\right)_{e} \otimes \mathbb{C}_{\nu}$. The sheaf $\mathcal{L}_{\chi, V}^{*}$ has a one-dimensional stalk at $e$, and any section of $\mathbb{C}_{\nu}$ has a numerical value at any particular $t \in A \cap G_{\mathbb{R}}$; thus $t$ acts on $\left(\mathcal{L}_{\chi, V}^{*}\right)_{e}$ by a scalar. We claim:

(9.43) via $\phi, t \in A \cap G_{\mathbb{R}}$ acts on $\left(\mathcal{L}_{\chi, V}^{*}\right)_{e}$ as the scalar $e^{\nu}(t) \frac{\chi_{F}(t)}{\left|e^{\left(\rho_{x}-\rho_{M, x}\right)}(t)\right|}$.

Secondly, we apply the fixed point formalism to the dualizing sheaf $\mathbb{D}_{V_{\mathbb{R}}^{+}}$. When we

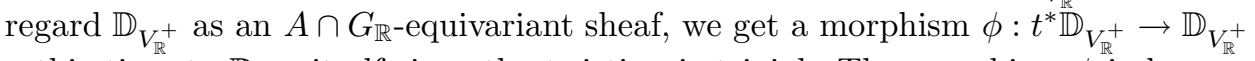
- this time to $\mathbb{D}_{V_{\mathbb{R}}^{+}}$itself since the twisting is trivial. The morphism $\phi$ induces a morphism $\phi_{t}$ on the stalk of the local cohomology sheaf at the fixed point $e$, in analogy to (5.23). We claim:

$$
\sum(-1)^{i} \operatorname{tr}\left(\phi_{t}: \mathcal{H}_{V^{\prime}}^{i}\left(\mathbb{D}_{V_{\mathbb{R}}^{+}}\right)_{e} \rightarrow \mathcal{H}_{V^{\prime}}^{i}\left(\mathbb{D}_{V_{\mathbb{R}}^{+}}\right)_{e}\right)=(-1)^{k_{x}}
$$

Together, (9.43)-(9.44) will imply (9.41), so we only need to verify these two assertions.

To clarify the reason for (9.43)-(9.44), let us look more generally at the case of a Lie group $L$ acting transitively on a manifold $M$. An $L$-equivariant local system $\mathcal{E}$ on $M$ is determined by the datum of a representation $\tau$ of the component group $\pi_{0}\left(L_{m}\right)$, of the isotropy group $L_{m}$ at some $m \in M$. On the other hand, the formalism of equivariant sheaves involves a distinguished isomorphism $\phi: a^{*} \mathcal{E} \rightarrow$ $p^{*} \mathcal{E}$ on $L \times M$. Since $L_{m}$ fixes $m$, both $\phi$ and its inverse $\phi^{-1}: p^{*} \mathcal{E} \rightarrow a^{*} \mathcal{E}$ induce maps $\phi, \phi^{-1}: L_{m} \rightarrow \operatorname{Aut}\left(\mathcal{E}_{m}\right)$, which are related to $\tau$ by

$$
\phi^{-1}(\ell)=\tau(\ell), \quad \text { for } \ell \in L_{m} .
$$

Indeed, $\phi_{\ell, m}$, for $(\ell, m) \in L \times M$, maps $\mathcal{E}_{\ell m}$ to $\mathcal{E}_{m}$, hence the cocycle condition on $\phi$ becomes

$$
\phi_{\ell_{2} \ell_{1}, m}^{-1}=\phi_{\ell_{2}, \ell_{1} m}^{-1} \circ \phi_{\ell_{1}, m}^{-1}
$$

whereas $\phi$ has the wrong variance to be multiplicative. The correspondence (9.45) is obtained by restricting $\phi^{-1}$ to $L_{m} \times\{m\}$.

We apply this discussion to the case $L=G_{\mathbb{R}} \times \mathfrak{h}$ and $M=\hat{S}$, the inverse image in $\hat{X}$ of the orbit $S$ to which we had associated the local system $\mathcal{L}_{\chi}$. Since we are treating $\mathcal{L}_{\chi}$ as a $G_{\mathbb{R}} \times \mathfrak{h}$-equivariant sheaf, we shall follow the conventions of (5.5)-(5.8) and use the symbol $\phi^{\prime}$ for the map which relates $\epsilon^{*} a^{*} \mathcal{L}_{\chi}^{*}$ to $\epsilon^{*} p^{*} \mathcal{L}_{\chi}^{*}$ :

$$
\phi^{\prime}: \epsilon^{*} a^{*} \mathcal{L}_{\chi}^{*} \rightarrow \epsilon^{*} p^{*} \mathcal{L}_{\chi}^{*},
$$

as in (5.6a). Then, as was argued in $\S 5$, the product $e^{\lambda-\rho} \phi^{\prime}$ descends to a map

$$
\phi=e^{\lambda-\rho} \phi^{\prime}: a^{*} \mathcal{L}_{\chi}^{*} \rightarrow p^{*} \mathcal{L}_{\chi}^{*} .
$$


In the construction of the local system $\mathcal{L}_{\chi}$ before the statement of Lemma 6.9 the action of the component group $\pi_{0}\left(\left(G_{\mathbb{R}} \times \mathfrak{h}\right)_{\hat{X}_{0}}\right)$ on the stalk of $\mathcal{L}_{\chi}$ at $\hat{x}^{0}$ was written as $\psi$, and was related to the character $\chi$ to which $\mathcal{L}_{\chi}$ is associated by the formula

$$
\psi=\tilde{\chi} e^{-(\lambda-\rho)},
$$

where $\tilde{\chi}$ is the lifting of $\chi$ from $T_{\mathbb{R}}$ to $\left(G_{\mathbb{R}}\right)_{x_{0}}-$ cf. (6.6a) and (6.8). According to (9.45), specialized to the present situation, $\psi$ coincides with the inverse of the character $\phi^{\prime}$, but the one corresponding to $\mathcal{L}_{\chi}$ rather than its dual as in (9.47a). Thus, with $\phi^{\prime}$ as in (9.47a), we get the equality $\psi=\left(\phi^{\prime}\right)^{*}\left(=\phi^{\prime}\right.$, when $\phi^{\prime}$ is viewed as a character on the fixed point set), hence

$$
\chi=\left.\left(\psi e^{\lambda-\rho}\right)\right|_{T_{\mathbb{R}}}=\left.\left(\phi^{\prime} e^{\lambda-\rho}\right)\right|_{T_{\mathbb{R}}}=\left.\phi\right|_{T_{\mathbb{R}}} .
$$

We restrict this identity further to $A \cap G_{\mathbb{R}}=A \cap T_{\mathbb{R}}$ and observe that

$$
\chi(t)=e^{\nu}(t) \frac{\chi_{F}(t)}{\left|e^{\left(\rho_{x}-\rho_{M, x}\right)}(t)\right|} \quad \text { for } t \in A \cap G_{\mathbb{R}} ;
$$

note: $\left.d \chi\right|_{\mathfrak{a}_{\mathbb{R}}}=\left.(\lambda-\rho)\right|_{\mathfrak{a}_{\mathbb{R}}}=\nu-\left.\left(\rho-\rho_{M}\right)\right|_{\mathfrak{a}_{\mathbb{R}}}$, from which (9.50) follows when $t \in\left(A \cap G_{\mathbb{R}}\right)^{0}$. But $\chi_{F}=\left.\chi\right|_{F}$, so (9.50) follows for every $t \in A \cap G_{\mathbb{R}}$. At this point, (9.49)-(9.50) imply (9.43).

The verification of (9.44) is simpler since the equivariant sheaf $\mathbb{D}_{V_{\mathbb{R}}^{+}}$is untwisted. In fact, (9.44) can be deduced directly from [KSa, 9.6.14]; however, the present instance of Kashiwara-Schapira's result is elementary so we shall argue directly. Concretely,

$$
\mathcal{H}_{V^{\prime}}^{*}\left(\mathbb{D}_{V_{\mathbb{R}}^{+}}\right)=o r_{V^{\prime} \cap V_{\mathbb{R}}^{+}}\left[\operatorname{dim}_{\mathbb{R}} V^{\prime} \cap V_{\mathbb{R}}^{+}\right]
$$

is the orientation sheaf of $V^{\prime} \cap V_{\mathbb{R}}^{+}$in degree $-\left(\operatorname{dim}_{\mathbb{R}} V^{\prime} \cap V_{\mathbb{R}}^{+}\right)$. The exponential map maps $\mathfrak{v}_{\mathbb{R}}^{+} \cap \mathfrak{n}^{\prime}(t, x)$ homeomorphically and $\tilde{T}_{\mathbb{R}^{-}}$equivariantly onto $V^{\prime} \cap V_{\mathbb{R}}^{+}$. Recall the condition (5.15) on the subset $\Psi^{\prime} \subset \Phi^{+}$. For $\alpha \in \Psi^{\prime}, \alpha \notin \Phi_{M}$, we have the following possibilities:

a) $\alpha \in \Psi^{\prime}, \alpha=-\bar{\alpha} \Longrightarrow \mathfrak{g}^{\alpha} \cap \mathfrak{v}_{\mathbb{R}}^{+} \cap \mathfrak{n}^{\prime}(t, x)=0$;

b) $\alpha \in \Psi^{\prime}, \alpha=\bar{\alpha} \Longrightarrow \operatorname{dim}_{\mathbb{R}}\left(\mathfrak{g}^{\alpha} \cap \mathfrak{v}_{\mathbb{R}}^{+} \cap \mathfrak{n}^{\prime}(t, x)\right)=1$;

c) $\alpha, \bar{\alpha} \in \Psi^{\prime}, \alpha \neq \pm \bar{\alpha} \Longrightarrow \operatorname{dim}_{\mathbb{R}}\left(\left(\mathfrak{g}^{\alpha} \oplus \mathfrak{g}^{\bar{\alpha}}\right) \cap \mathfrak{v}_{\mathbb{R}}^{+} \cap \mathfrak{n}^{\prime}(t, x)\right)=2$;

d) $\alpha \in \Psi^{\prime}, \bar{\alpha} \notin \Psi^{\prime}, \alpha \neq \pm \bar{\alpha} \Longrightarrow\left(\mathfrak{g}^{\alpha} \oplus \mathfrak{g}^{\bar{\alpha}}\right) \cap \mathfrak{v}_{\mathbb{R}}^{+} \cap \mathfrak{n}^{\prime}(t, x)=0 ;$

moreover, $\mathfrak{v}_{\mathbb{R}}^{+} \cap \mathfrak{n}^{\prime}(t, x)$ splits into the direct sum of the intersections appearing on the right in (9.52). Thus

$$
\operatorname{dim}_{\mathbb{R}}\left(\mathfrak{v}_{\mathbb{R}}^{+} \cap \mathfrak{n}^{\prime}(t, x)\right) \equiv \#\left\{\alpha \in \Psi^{\prime} \mid \alpha=\bar{\alpha}\right\} \quad(\bmod 2) .
$$

In the situation (9.52c), $t$ acts on $\mathfrak{g}^{\alpha} \oplus \mathfrak{g}^{\bar{\alpha}}$ with eigenvalues $e^{\alpha}(t), e^{\bar{\alpha}}(t)$, and hence preserves the orientation of $\left(\mathfrak{g}^{\alpha} \oplus \mathfrak{g}^{\bar{\alpha}}\right) \cap \mathfrak{v}_{\mathbb{R}}^{+} \cap \mathfrak{n}^{\prime}(t, x)$. On the other hand, in the situation (9.52b), $t$ preserves or reverses the orientation of $\mathfrak{g}^{\alpha} \cap \mathfrak{v}_{\mathbb{R}}^{+} \cap \mathfrak{n}^{\prime}(t, x)$ depending on the sign of $e^{\alpha}(t)$. Hence

$$
t \text { preserves or reverses the orientation of } \mathfrak{v}_{\mathbb{R}}^{+} \cap \mathfrak{n}^{\prime}(t, x)
$$
depending on the parity of $\#\left\{\alpha \in \Psi^{\prime} \mid \alpha=\bar{\alpha}, e^{\alpha}(t)<0\right\}$. 
Finally, since complex roots in $\Phi-\Phi_{M}$ occur in pairs, and since $t$ must be regular,

$$
\begin{aligned}
k_{x} & =\#\left\{\alpha \in \Phi^{+}-\Phi_{M}^{+} \mid 0<e^{\alpha}(t)<1\right\} \\
& \equiv \#\left\{\alpha \in \Psi^{\prime} \mid \alpha=\bar{\alpha}, e^{\alpha}(t)>0\right\} \quad(\bmod 2) .
\end{aligned}
$$

In view of $(9.51), \phi_{t}: \mathcal{H}_{V^{\prime}}^{*}\left(\mathbb{D}_{V_{\mathbb{R}}^{+}}\right)_{e} \rightarrow \mathcal{H}_{V^{\prime}}^{*}\left(\mathbb{D}_{V_{\mathbb{R}}^{+}}\right)_{e}$ is the geometric action of $t$ on the orientation of $\mathfrak{v}_{\mathbb{R}}^{+} \cap \mathfrak{n}^{\prime}(t, x)$ (in degree $\left.-\operatorname{dim}_{\mathbb{R}} \mathfrak{v}_{\mathbb{R}}^{+} \cap \mathfrak{n}^{\prime}(t, x)\right)$. At this point (9.53)(9.55) imply (9.44), and that completes the proof.

\section{INTERTWINING FUNCTORS}

So far, we have verified the main theorems for all standard sheaves associated to maximally real orbits. In the present section we shall use the mechanism of intertwining functors to extend the results to standard sheaves attached to all orbits; as was argued in $\S 6$, this will complete our proofs of Theorems $3.8,5.12,5.24$, and 5.27 .

We begin by recalling certain facts about the orbit structure. Let $T_{\mathbb{R}} \subset G_{\mathbb{R}}$ be a Cartan subgroup, $x_{0} \in X$ a fixed point of $T_{\mathbb{R}}$, and $\tau_{x_{0}}: \mathfrak{t} \rightarrow \mathfrak{h}$ the corresponding isomorphism. As was mentioned in $\S 6$, these data determine a $G_{\mathbb{R}}$-orbit

$$
S_{0}=S\left(T_{\mathbb{R}}, \tau_{x_{0}}\right),
$$

and the correspondence between pairs $\left(T_{\mathbb{R}}, \tau_{x_{0}}\right)$ becomes bijective when $T_{\mathbb{R}}$ is taken modulo $G_{\mathbb{R}}$-conjugacy and, once $T_{\mathbb{R}}$ is specified, $\tau_{x_{0}}$ modulo $N_{G_{\mathbb{R}}}\left(T_{\mathbb{R}}\right)$-conjugacy. Let us consider a particular orbit $S_{0}$ as in (10.1). The isomorphism $\tau_{x_{0}}$ identifies the universal root system $\Phi$ with the concrete root system $\Phi(\mathfrak{g}, \mathfrak{t})$. We use this identification to transfer the universal positive root system $\Phi^{+}$to a positive root system $\Phi_{S_{0}}^{+}(\mathfrak{g}, \mathfrak{t})$ in $\Phi(\mathfrak{g}, \mathfrak{t})$; this positive root system does depend only on the orbit, since $S_{0}$ determines $\left(T_{\mathbb{R}}, \tau_{x_{0}}\right)$ up to conjugacy. Recall the notions of real, imaginary, and complex root, which were defined in $\S 9$. The integer

$$
c\left(S_{0}\right)=\#\left\{\alpha \in \Phi^{+} \mid \tau_{x_{0}}^{*} \alpha \text { is complex and } \overline{\tau_{x_{0}}^{*} \alpha} \in-\Phi_{S_{0}}^{+}(\mathfrak{g}, \mathfrak{t})\right\}
$$

measures the extent to which $S_{0}$ fails to be maximally real: if $c\left(S_{0}\right)=0$, it is maximally real, so we do not need to deal with this case any further.

Let us suppose then that $c\left(S_{0}\right)>0$. According to (9.1), there must exist at least one simple root $\alpha \in \Phi^{+}$such that $\tau_{x_{0}}^{*} \alpha \in \Phi_{S_{0}}^{+}(\mathfrak{g}, \mathfrak{t})$ is complex and has a negative complex conjugate $\overline{\tau_{x_{0}}^{*} \alpha} \in-\Phi_{S_{0}}^{+}(\mathfrak{g}, \mathfrak{t})$. Let us fix such a simple root $\alpha$. It determines a $G$-equivariant fibration

$$
X \stackrel{\pi}{\longrightarrow} X_{\alpha} \quad \text { with fiber } \mathbb{P}^{1}
$$

over the generalized flag variety $X_{\alpha}$. The Cartan subgroup $T_{\mathbb{R}}$ has exactly two fixed points on the fiber $\pi^{-1}\left(\pi\left(x_{0}\right)\right)$; these correspond to the fixed points of a Cartan subgroup of the group $S L(2, \mathbb{C})$ acting on $\mathbb{P}^{1}$. One of the two fixed points is $x_{0}$. We call the second fixed point $x_{1}$. Let $S_{1}$ be the $G_{\mathbb{R}}$-orbit through $x_{1}$. Then

$$
\begin{aligned}
& \text { a) } \pi^{-1}\left(\pi\left(S_{0}\right)\right)=S_{0} \cup S_{1} ; \\
& \text { b) } \tau_{x_{1}} \circ \tau_{x_{0}}^{-1}: \mathfrak{h} \rightarrow \mathfrak{h} \text { is reflection about the root } \alpha ; \\
& \text { c) } c\left(S_{1}\right)=c\left(S_{0}\right)-1,
\end{aligned}
$$

as is shown in [HMSW2], [S4], for example.

Let $\mathcal{F}_{0} \in \mathrm{D}_{G_{\mathbb{R}}}(X)_{-\lambda}$ be a standard sheaf attached to the orbit $S_{0}$. We had argued in $\S 6$ that $\mathcal{F}_{0}$ is the direct image $R j_{0 *} \mathcal{L}_{\chi_{0}}$ of an irreducible, $G_{\mathbb{R}}$-equivariant 
local system $\mathcal{L}_{\chi_{0}}$ on the orbit $S_{0}$. This local system corresponds to the datum of a character

$$
\chi_{0}: T_{\mathbb{R}} \longrightarrow \mathbb{C}^{*}, \quad \text { with } d \chi_{0}=\tau_{x_{0}}^{*}(\lambda-\rho) .
$$

We define a new character $\chi_{1}$ by the formula

$$
\chi_{1}=e^{-\alpha_{x_{1}}} \chi_{0}: T_{\mathbb{R}} \longrightarrow \mathbb{C}^{*},
$$

where $\alpha_{x_{1}}=\tau_{x_{1}}^{*} \alpha$, as before. Then, because of (10.4b),

$$
d \chi_{1}=-\tau_{x_{1}}^{*} \alpha+\tau_{x_{0}}^{*}(\lambda-\rho)=\tau_{x_{1}}^{*}\left(-\alpha+s_{\alpha}(\lambda-\rho)\right),
$$

hence

$$
d \chi_{1}=\tau_{x_{1}}^{*}\left(s_{\alpha} \lambda-\rho\right) .
$$

Since $\chi_{1}$ satisfies this condition, it determines an irreducible $G_{\mathbb{R}}$-equivariant local system $\mathcal{L}_{\chi_{1}}$ on $S_{1}$, whose direct image is a standard sheaf $\mathcal{F}_{1} \in \mathrm{D}_{G_{\mathbb{R}}}(X)_{-s_{\alpha} \lambda}$ attached to the orbit $S_{1}$. We shall argue by induction on the integer (10.2), and thus assume

$$
\text { Theorems 3.8, 5.12, 5.24, and } 5.27 \text { are satisfied by the sheaf } \mathcal{F}_{1} \text {. }
$$

We shall deduce the validity of these theorems for $\mathcal{F}_{0}$, which will then imply the theorems in full generality.

The two standard sheaves $\mathcal{F}_{0}, \mathcal{F}_{1}$ are related geometrically by an intertwining functor. Intertwining functors were introduced by Beilinson-Bernstein [BB2] in the context of $\mathcal{D}$-modules. Their formalism carries over readily to the setting of constructible (untwisted) sheaves on $X$ - see [SV4]. In the case of twisted sheaves on $X$, the construction of intertwining functors takes place on the generalized flag variety; this was worked out by Beilinson-Bernstein in an earlier, preprint version of [BB3]. Here we shall discuss their construction, in slightly modified form, in the case we need: the intertwining functor corresponding to the simple root $\alpha$.

Following the conventions in [SV4], we let $Y_{\alpha}$ denote the variety of pairs $\left(x^{\prime}, x^{\prime \prime}\right)$ in $X \times X$ in relative position $s_{\alpha}$, and $p, q$ for the natural projections

$$
X \stackrel{p}{\longleftarrow} Y_{\alpha} \stackrel{q}{\longrightarrow} X
$$

to the two factors. Both are fibrations with fiber $\mathbb{C}$. We shall put (10.8) into a commutative diagram

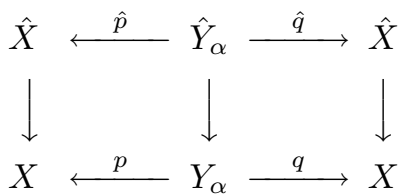

with certain properties that we shall explain next. Recall that the two outer vertical arrows exhibit $\hat{X}$ as principal $H$-bundle over $X$. To begin with,

$$
\hat{Y}_{\alpha} \longrightarrow Y_{\alpha} \text { is a principal } H \text {-bundle . }
$$

Thus $H$ operates on the three spaces in the top row in (10.9). With respect to these actions,

$$
\hat{p}(h \cdot \hat{y})=h \cdot \hat{p}(\hat{y}) \quad \text { and } \quad \hat{q}(h \cdot \hat{y})=s_{\alpha}(h) \cdot \hat{q}(\hat{y})
$$

for all $\hat{y} \in \hat{Y}_{\alpha}$ and $h \in H$. Lastly, 
These are the formal properties that will matter to us.

We shall not phrase our construction of the commutative diagram (10.9) in invariant terms, though this could be done along the lines of the discussion of the enhanced flag variety in $\S 2$. We make the identifications

$$
X=G / T N, \quad \hat{X}=G / N,
$$

where $T$ is a concrete Cartan subgroup, $N$ a maximal unipotent subgroup normalized by $T$. By letting $N$ correspond to the negative roots as usual, we obtain an explicit identification

$$
H \cong T
$$

between the concrete Cartan $T$ and the universal Cartan $H$. In the construction of the diagram (10.9), we shall think of (10.11b) as an equality, and accordingly shall identify the root system $\Phi(\mathfrak{g}, \mathfrak{t})$ with the universal root system $\Phi$.

We choose a particular representative $s$ for the Weyl reflection about the simple root $\alpha$,

$$
s \in N_{G}(T), \quad \text { so that } \operatorname{Ad} s \text { induces } s_{\alpha} \in W,
$$

and set

$$
\mathfrak{n}^{\alpha}=\mathfrak{n} \cap(\operatorname{Ad} s) \mathfrak{n}=\bigoplus_{\beta \in \Phi^{+}, \beta \neq \alpha} \mathfrak{g}^{-\beta}, \quad N^{\alpha}=\exp \left(\mathfrak{n}^{\alpha}\right) .
$$

The Cartan subgroup $T$ normalizes $N^{\alpha}=N \cap s N s^{-1}$. The group $T N^{\alpha}$ is precisely the simultaneous stabilizer of the identity coset and its $s$-translate in $G / T N=X$, so $G / T N^{\alpha}$ is the $G$-orbit through $(e B, s B) \in G / T N \times G / T N=X \times X-$ in other words, $G / T N^{\alpha}$ is the variety $Y_{\alpha}$ of pairs $\left(x^{\prime}, x^{\prime \prime}\right)$ in $X \times X$ in relative position $s_{\alpha}$ :

$$
Y_{\alpha}=G / T N^{\alpha}
$$

in terms of this identification, the projections $p, q: Y_{\alpha}=G / T N^{\alpha} \rightarrow G / T N=X$ are given by

$$
p\left(g T N^{\alpha}\right)=g T N, \quad q\left(g T N^{\alpha}\right)=g s T N .
$$

At this point it is a simple matter to finish the construction of the commutative diagram (10.9). We set

$$
\begin{gathered}
\hat{Y}_{\alpha}=G / N^{\alpha}, \\
\hat{p}\left(g N^{\alpha}\right)=g N, \quad \hat{q}\left(g N^{\alpha}\right)=g s N .
\end{gathered}
$$

The commutativity of the diagram and the properties (10.10a-c) are readily verified.

The Weyl reflection $s_{\alpha}$ induces an intertwining functor $I_{\alpha}$ from the (untwisted) bounded derived category of sheaves on $X$ to itself; for the discussion of the untwisted case, we shall rely on $[\mathrm{SV} 4, \S 7]$. Analogously we define a twisted intertwining functor

$$
\hat{I}_{\alpha}: \mathrm{D}_{G_{\mathbb{R}}}(X)_{-\lambda} \longrightarrow \mathrm{D}_{G_{\mathbb{R}}}(X)_{-s_{\alpha} \lambda}, \quad \hat{I}_{\alpha}(\mathcal{F})=R \hat{q}_{*} \hat{p}^{*}(\mathcal{F})[1] .
$$

By definition, $\hat{I}_{\alpha}$ operates on the category of $G_{\mathbb{R}} \times \mathfrak{h}$-equivariant sheaves. Because of (10.10b), it sends $(-\lambda-\rho)$-monodromic sheaves to $s_{\alpha}(-\lambda-\rho)$-monodromic sheaves. But $s_{\alpha}(-\lambda-\rho)$ differs from $-s_{\alpha} \lambda-\rho$ by the root $\alpha$, and the monodromicity condition depends only on the twisting parameter modulo the weight lattice. Thus $\hat{I}_{\alpha}$ affects the twisting as claimed in (10.16). 
10.17 Lemma. The intertwining functor $\hat{I}_{\alpha}$ maps the standard sheaf $\mathcal{F}_{1}$ to $\mathcal{F}_{0}[1]$, the standard sheaf $\mathcal{F}_{0}$ with a shift in degree.

Proof. By equivariance, $\hat{I}_{\alpha}\left(\mathcal{F}_{1}\right)$ is supported on a union of $G_{\mathbb{R}} \times H$-orbits in $\hat{X}$. Every such orbit is the inverse image $\hat{S}$ of a unique $G_{\mathbb{R}}$-orbit $S \subset X$. Let then $j: \hat{S} \hookrightarrow \hat{X}$ be the inclusion of an orbit. We shall show

$$
j ! \hat{I}_{\alpha}\left(\mathcal{F}_{1}\right)= \begin{cases}0 & \text { if } \hat{S} \neq \hat{S}_{0} \\ \mathcal{L}_{\chi_{0}}[1] & \text { if } \hat{S}=\hat{S}_{0}\end{cases}
$$

Let us argue first that (10.18) implies the conclusion of the lemma. We apply Verdier duality, which reverses the roles of stars and shrieks: to establish that $\mathcal{G}=\mathbb{D} \hat{I}_{\alpha}\left(\mathcal{F}_{1}\right)$ is the lower shriek extension (i.e., extension by zero) of a sheaf $\mathcal{L}$ on the orbit $\hat{S}_{0}$, it manifestly suffices to check that it restricts to $\mathcal{L}$ on $S_{0}$, and that its restriction to all the other orbits is zero.

To verify (10.18), we put the inclusions $j: \hat{S} \hookrightarrow \hat{X}, j_{1}: \hat{S}_{1} \hookrightarrow \hat{X}$ into the commutative diagram

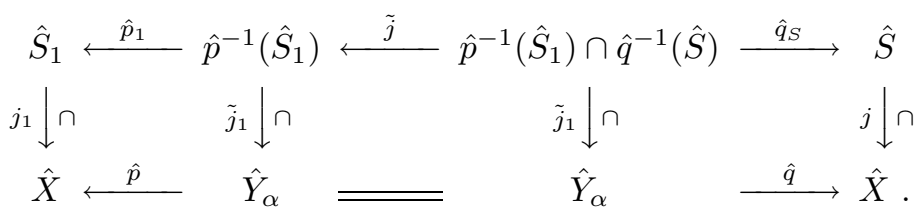

We need to identify

$$
j ! \hat{I}_{\alpha}\left(\mathcal{F}_{1}\right)=j^{!} R \hat{q}_{*} \hat{p}^{*} R j_{1 *}\left(\mathcal{L}_{\chi_{1}}\right)[1] .
$$

First we apply base change in the square on the left:

$$
\hat{p}^{*} R j_{1_{*}}\left(\mathcal{L}_{\chi_{1}}\right)=R \tilde{j}_{1 *} \hat{p}_{1}^{*}\left(\mathcal{L}_{\chi_{1}}\right) .
$$

Next, we consider the subdiagram with arrows $\tilde{j}, \hat{q} \circ \tilde{j}_{1}, \hat{q}_{S}$, and $j$, which is a Cartesian square. Thus, by base change,

$$
j^{!} R\left(\hat{q} \circ \tilde{j}_{1}\right)_{*}\left(\hat{p}_{1}^{*} \mathcal{L}_{\chi_{1}}\right)=R \hat{q}_{S_{*}} \tilde{j}^{!}\left(\hat{p}_{1}^{*} \mathcal{L}_{\chi_{1}}\right) .
$$

Combining (10.20a-c), we find

$$
j ! \hat{I}_{\alpha}\left(\mathcal{F}_{1}\right)=R \hat{q}_{S_{*}} \tilde{j}^{!}\left(\hat{p}_{1}^{*} \mathcal{L}_{\chi_{1}}\right)[1] .
$$

We claim:

$$
\hat{q}: \hat{p}^{-1} \hat{S}_{1} \longrightarrow \hat{S}_{0} \quad \text { is a }\left(G_{\mathbb{R}} \times H\right) \text {-equivariant isomorphism, }
$$

relative to the natural action of $G_{\mathbb{R}}$ on the two spaces, the natural action of $H$ on $\hat{p}^{-1} \hat{S}_{1}$, and the action of $H$ on $\hat{S}_{0}$ obtained by composing the natural action with the Weyl reflection $s_{\alpha}-$ cf. (10.10b). Let us assume this for the moment.

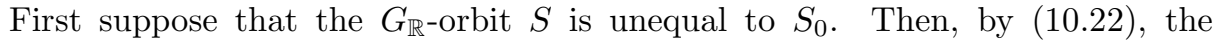
intersection $\hat{p}^{-1}\left(\hat{S}_{1}\right) \cap \hat{q}^{-1}(\hat{S})$ is empty. This intersection appears in the diagram (10.19). Hence, in (10.21), the right hand side is the direct image of a sheaf on the empty set - the zero sheaf. Conclusion: $j ! \hat{I}_{\alpha}\left(\mathcal{F}_{1}\right)=0$ when $S \neq S_{0}$.

Now we suppose that $S=S_{0}$. Then, in (10.19), $\hat{p}^{-1}\left(\hat{S}_{1}\right) \cap \hat{q}^{-1}\left(\hat{S}_{0}\right)$ coincides with $\hat{p}^{-1}\left(\hat{S}_{1}\right), \tilde{j}$ reduces to the identity, and $\hat{q}_{S}$ is the isomorphism (10.22). Thus, in the present situation,

$$
j ! \hat{I}_{\alpha}\left(\mathcal{F}_{1}\right)=\hat{q}_{S *} \hat{p}_{1}^{*} \mathcal{L}_{\chi_{1}}[1]
$$


is - up to a shift in degree - the pullback to $\hat{S}_{0} \simeq \hat{p}^{-1}\left(\hat{S}_{1}\right)$ of a $\left(G_{\mathbb{R}} \times \mathfrak{h}\right)$-equivariant local system under the $\left(G_{\mathbb{R}} \times \mathfrak{h}\right)$-equivariant map $\hat{p}_{1}$. As explained in $\S 6$, these equivariant local systems are specified by characters of the Cartan subgroup $T_{\mathbb{R}}$, which fixes the two base points $x_{0}, x_{1}$. The correspondence between equivariant local systems and characters involves the identification between the complexification $T$ of $T_{\mathbb{R}}$ and the universal Cartan $H$. The two identifications $T \cong H$ determined by the base points $x_{0}, x_{1}$ are related by the Weyl reflection $s_{\alpha}$. On the other hand, the isomorphism $\hat{S}_{0} \simeq \hat{p}^{-1}\left(\hat{S}_{1}\right)$ is $H$-equivariant only when the $H$-action on one of the two spaces is twisted by $s_{\alpha}$. These two occurrences of $s_{\alpha}$ cancel. It follows that $j ! \hat{I}_{\alpha}\left(\mathcal{F}_{1}\right)[-1]$ is the equivariant local system attached to the character $\chi_{1}=e^{-\alpha} \chi_{0}$; note: our labeling of the twists involves the shift by $\rho$, and $s_{\alpha} \rho=\rho-\alpha$ - this accounts for the factor $e^{-\alpha}$ in the relation between $\chi_{0}$ and $\chi_{1}$.

This completes the proof of the lemma, except the verification of (10.22). The orbits $\hat{S}_{0}, \hat{S}_{1}$ fiber as principal $H$-bundles over the $G_{\mathbb{R}}$-orbits $S_{0}$ and $S_{1}$, respectively. Thus (10.22) follows formally from (10.10b) and

$$
q: p^{-1} S_{1} \stackrel{\sim}{\longrightarrow} S_{0} \text { is a } G_{\mathbb{R}} \text {-equivariant isomorphism . }
$$

This assertion, in turn, reduces to the following statement: let $F \cong \mathbb{P}^{1}$ be the fiber of the fibration (10.3) which contains $x_{0}$ and $x_{1}$; then

$$
\begin{aligned}
& \text { a) } F \cap S_{1}=\left\{x_{1}\right\} ; \\
& \text { b) } F \cap S_{0}=F-\left\{x_{1}\right\} .
\end{aligned}
$$

This, in effect, is a paraphrase of (10.4).

10.26 Lemma. $\Theta\left(\mathcal{F}_{1}\right)=-\Theta\left(\mathcal{F}_{0}\right)$.

This fact is established, explicitly or implicitly, in various places. See for example [HMSW2], [S4].

The discussion in this section up to this point is relevant to the proof both of the integral formula 3.8 and the fixed point theorems 5.12, 5.24, and 5.27. We now turn specifically to the integral formula. In [SV4] we remarked that the (untwisted) intertwining functors on the bounded derived category of (semi-algebraically) constructible sheaves,

$$
I_{w}: \mathrm{D}_{c}^{b}(X) \longrightarrow \mathrm{D}_{c}^{b}(X), \quad w \in W
$$

induce an action of $W$ on $\mathcal{L}^{+}(X)$, the group of $\mathbb{R}^{+}$-conical, semi-algebraic Lagrangian cycles in $T^{*} X$ so that the map CC : $\mathrm{D}_{c}^{b}(X) \rightarrow \mathcal{L}^{+}(X)$ becomes $W$ equivariant.

10.28 Lemma. $\operatorname{CC}\left(\hat{I}_{\alpha} \mathcal{F}\right)=I_{\alpha} \operatorname{CC}(\mathcal{F})$, for any $\lambda \in \mathfrak{h}^{*}$ and $\mathcal{F} \in \mathrm{D}_{G_{\mathbb{R}}}(X)_{\lambda}$.

Proof. We think of $\mathcal{F}$ as an element of the $K$-group of $\mathrm{D}_{c}^{b}\left(S h_{X, \lambda}\right)$. This $K$-group is generated by locally constant twisted sheaves on contractible closed subsets of $X$. For sheaves of this special type, the assertion of the lemma follows from the properties of the diagram (10.9).

Recall the definition of the group $\mathrm{H}_{2 n}^{\text {inf }}\left(T_{G_{\mathbb{R}}}^{*} X, \mathbb{Z}\right)$, which contains the characteristic cycle $\operatorname{CC}\left(\mathcal{F}_{1}\right)$, and of the differential forms $\sigma, \tau_{\lambda}$ in $\S 3$.

10.29 Lemma. For all $\phi \in C_{c}^{\infty}\left(\mathfrak{g}_{\mathbb{R}}\right)$ and $\lambda \in \mathfrak{h}^{*}$,

$$
\int_{I_{\alpha} \mathrm{CC}\left(\mathcal{F}_{1}\right)} \mu_{\lambda}^{*} \hat{\phi}\left(-\sigma+\pi^{*} \tau_{\lambda}\right)^{n}=\int_{\mathrm{CC}\left(\mathcal{F}_{1}\right)} \mu_{s_{\alpha} \lambda}^{*} \hat{\phi}\left(-\sigma+\pi^{*} \tau_{s_{\alpha} \lambda}\right)^{n}
$$


Before giving the proof, let us remark that Lemmas 10.17, 10.28, and 10.29 imply

$$
\begin{aligned}
& \frac{1}{(2 \pi i)^{n} n !} \int_{\mathrm{CC}\left(\mathcal{F}_{0}\right)} \mu_{\lambda}^{*} \hat{\phi}\left(-\sigma+\pi^{*} \tau_{\lambda}\right)^{n}= \\
& =-\frac{1}{(2 \pi i)^{n} n !} \int_{\mathrm{CC}\left(\mathcal{F}_{1}\right)} \mu_{s_{\alpha} \lambda}^{*} \hat{\phi}\left(-\sigma+\pi^{*} \tau_{s_{\alpha} \lambda}\right)^{n}
\end{aligned}
$$

for any $\phi \in C_{c}^{\infty}\left(\mathfrak{g}_{\mathbb{R}}\right)$. The induction hypothesis identifies the right hand side of (10.30) as the integral of $\phi$ against the distribution $-\theta\left(\mathcal{F}_{1}\right)$. Thus, by 10.26 ,

$$
\frac{1}{(2 \pi i)^{n} n !} \int_{\mathrm{CC}\left(\mathcal{F}_{0}\right)} \mu_{\lambda}^{*} \hat{\phi}\left(-\sigma+\pi^{*} \tau_{\lambda}\right)^{n}=\int_{\mathfrak{g}_{\mathbb{R}}} \theta\left(\mathcal{F}_{0}\right) \phi d x
$$

This is the assertion of Theorem 3.8 for the sheaf $\mathcal{F}_{0}$, thus completing our inductive proof of the theorem.

Proof of Lemma 10.29. By Proposition 3.7, we may as well assume that $\lambda$ is regular. According to [SV4, theorem 9.1], the $W$-action (10.27) agrees with the geometric action described in [SV4, §8], which was originally defined by Rossmann [R3]. The path

$$
\gamma(t)=t s_{\alpha} \lambda, \quad 0 \leq t \leq 1,
$$

satisfies the hypothesis of [SV4, lemma 9.4]. Thus

$$
I_{\alpha} \mathrm{CC}\left(\mathcal{F}_{1}\right)=\lim _{t \rightarrow 0^{+}}\left(\mu_{t \lambda}^{-1} \circ \mu_{t s_{\alpha} \lambda}\right) \mathrm{CC}\left(\mathcal{F}_{1}\right)=-\partial C+\left(\mu_{\lambda}^{-1} \circ \mu_{s_{\alpha} \lambda}\right) \operatorname{CC}\left(\mathcal{F}_{1}\right),
$$

where $C$ is the semi-algebraic chain

$$
C=\left\{\left(\mu_{t \lambda}^{-1} \circ \mu_{t s_{\alpha} \lambda}\right) \mathrm{CC}\left(\mathcal{F}_{1}\right) \mid 0 \leq t \leq 1\right\}
$$

for the relation between the boundary of the chain (10.32b) and the limit (10.32a) and the convention for orienting the chain, see [SV4, §3]. The chain $C$ satisfies the hypothesis of Lemma 3.19 because the curve (10.23) is compact. We conclude

$$
\begin{gathered}
\int_{I_{\alpha} \operatorname{CC}\left(\mathcal{F}_{1}\right)} \mu_{\lambda}^{*} \hat{\phi}\left(-\sigma+\pi^{*} \tau_{\lambda}\right)^{n}=\int_{\left(\mu_{\lambda}^{-1} \circ \mu_{s_{\alpha} \lambda}\right) \operatorname{CC}\left(\mathcal{F}_{1}\right)} \mu_{\lambda}^{*} \hat{\phi}\left(-\sigma+\pi^{*} \tau_{\lambda}\right)^{n} \\
=\int_{\operatorname{CC}\left(\mathcal{F}_{1}\right)} \mu_{s_{\alpha} \lambda}^{*} \hat{\phi}\left(\mu_{\lambda}^{-1} \circ \mu_{s_{\alpha} \lambda}\right)^{*}\left(-\sigma+\pi^{*} \tau_{\lambda}\right)^{n}
\end{gathered}
$$

The complex coadjoint orbit $\Omega_{\lambda}$ and the canonical symplectic form $\sigma_{\lambda}$ on it depend only on the $W$-orbit of $\lambda$, not on $\lambda$ itself. Hence, by Proposition 3.3,

$$
\mu_{\lambda}^{*} \sigma_{\lambda}=-\sigma+\pi^{*} \tau_{\lambda} \text { and } \mu_{s_{\alpha} \lambda}^{*} \sigma_{\lambda}=-\sigma+\pi^{*} \tau_{s_{\alpha} \lambda}
$$

which implies $\left(\mu_{\lambda}^{-1} \circ \mu_{s_{\alpha} \lambda}\right)^{*}\left(-\sigma+\pi^{*} \tau_{\lambda}\right)=-\sigma+\pi^{*} \tau_{s_{\alpha} \lambda}$. In view of (10.33), this completes the proof of Lemma 10.29.

Let us carry out the induction step for the fixed point formulas 5.24; we had argued earlier that 5.24 implies also Theorems 5.12 and 5.27. According to the induction hypothesis (10.7), we know the assertion of 5.24 for the particular sheaf $\mathcal{F}_{1}$. We must establish it also for $\mathcal{F}_{0}=\hat{I}_{\alpha} \mathcal{F}_{1}[-1]$. As in the statement of 5.24 we fix a regular semisimple element $t \in G_{\mathbb{R}}{ }^{\prime}$ and a fixed point $x \in X$ of $t$. Then $x$ is a fixed point also of the Cartan subgroup $\tilde{T}_{\mathbb{R}}=N_{G_{\mathbb{R}}}(t)$. Let us denote the sections $c_{t, x}$ corresponding to $\mathcal{F}_{0}, \mathcal{F}_{1}$ by $c_{t, x}\left(\mathcal{F}_{0}\right), c_{t, x}\left(\mathcal{F}_{1}\right)$. Note that $\mathcal{F}_{0} \in \mathrm{D}_{G_{\mathbb{R}}}(X)_{-\lambda}$, whereas $\mathcal{F}_{1} \in \mathrm{D}_{G_{\mathbb{R}}}(X)_{-s_{\alpha} \lambda}$. Hence $c_{t, x}\left(\mathcal{F}_{0}\right)$ is a (local) section of $\mathbb{C}_{\lambda}$ - i.e., a multiple of a 
branch of the multiple valued function $e^{\lambda_{x}-\rho_{x}}$ - and $c_{t, x}\left(\mathcal{F}_{1}\right)$ is a section of $\mathbb{C}_{s_{\alpha} \lambda}-$ i.e., a multiple of a branch of the multiple valued function $e^{s_{\alpha} \lambda_{x}-\rho_{x}}$. Comparing the local expressions (4.11) for the two invariant eigendistributions $\Theta\left(\mathcal{F}_{0}\right)$ and $\Theta\left(\mathcal{F}_{1}\right)$, and taking into account Lemma 10.26, we find

$$
e^{\alpha_{x}} c_{t, x}\left(\mathcal{F}_{0}\right)=c_{t, s_{\alpha} x}\left(\mathcal{F}_{1}\right) \quad \text { for all } x \in X^{\tilde{T}_{\mathbb{R}}}
$$

Here $s_{\alpha}$ is shorthand notation for the point obtained as follows: we identify the abstract Weyl group $W$ with the concrete Weyl group $W(G, \tilde{T})$ via $\tau_{x}$; via this identification, $W$ permutes the fixed points of $\tilde{T}$.

Theorem 5.24 expresses the two constants $c_{t, x}\left(\mathcal{F}_{0}\right)$ and $c_{t, s_{\alpha} x}\left(\mathcal{F}_{1}\right)$ in $(10.35)$ as fixed point expressions applied not to the sheaves $\mathcal{F}_{0}$ and $\mathcal{F}_{1}$ themselves, but rather their duals $\mathcal{G}_{0}=\mathbb{D} \mathcal{F}_{0}, \mathcal{G}_{1}=\mathbb{D} \mathcal{F}_{1}$. Let us denote the fixed point expressions in the second line of 5.24 by

$$
\begin{gathered}
d_{t, x}\left(\mathcal{G}_{0}\right)=\sum(-1)^{i} \operatorname{tr}\left(\phi_{t}: \mathcal{H}_{\{x\}}^{i}\left(\left.\mathcal{G}_{0}(x)\right|_{N^{\prime \prime}(t, x)}\right) \rightarrow \mathcal{H}_{\{x\}}^{i}\left(\left.\mathcal{G}_{0}(x)\right|_{N^{\prime \prime}(t, x)}\right) \otimes \mathbb{C}_{\lambda}\right), \\
d_{t, x}\left(\mathcal{G}_{1}\right)=\sum(-1)^{i} \operatorname{tr}\left(\phi_{t}: \mathcal{H}_{\{x\}}^{i}\left(\left.\mathcal{G}_{1}(x)\right|_{N^{\prime \prime}(t, x)}\right) \rightarrow \mathcal{H}_{\{x\}}^{i}\left(\left.\mathcal{G}_{1}(x)\right|_{N^{\prime \prime}(t, x)}\right) \otimes \mathbb{C}_{s_{\alpha} \lambda}\right) ;
\end{gathered}
$$

note that these constants have a different meaning from the $d_{t, x}$ in $\S 5$. Our induction hypothesis is contained in the equation

$$
d_{t, x}\left(\mathcal{G}_{1}\right)=c_{t, x}\left(\mathcal{F}_{1}\right)
$$

for all regular $t$ and all fixed points $x$ of $t$. The conclusion we want is

$$
d_{t, x}\left(\mathcal{G}_{0}\right)=c_{t, x}\left(\mathcal{F}_{0}\right)
$$

again for all $t$ and $x$. Dualizing the relation $\hat{I}_{\alpha} \mathcal{F}_{1}=\mathcal{F}_{0}[1]-$ cf. (10.16) and Lemma 10.17 - we find

$$
\hat{J}_{\alpha} \mathcal{G}_{1}=\mathcal{G}_{0}[-1], \quad \text { where } \hat{J}_{\alpha}=R \hat{q}_{!} \hat{p}^{*}[1] .
$$

In view of (10.35) and (10.37), the inductive conclusion (10.36b) will follow from the identity

$$
e^{\alpha_{x}} d_{t, x}\left(\hat{J}_{\alpha} \mathcal{G}\right)=-d_{t, s_{\alpha} x}(\mathcal{G})
$$

for $\mathcal{G}=\mathcal{G}_{1}$; the shift by $[-1]$ accounts for the minus sign. Our remaining task is to verify (10.38) for an arbitrary twisted sheaf $\mathcal{G} \in \mathrm{D}_{G_{\mathbb{R}}}(X)_{\lambda}$.

10.39 Lemma. In the $K$-group $K\left(\mathrm{D}_{G_{\mathbb{R}}}(X)_{\lambda}\right)$, the square $\hat{J}_{\alpha} \circ \hat{J}_{\alpha}$ of the operator $\hat{J}_{\alpha}$ coincides with the identity.

In the untwisted case this follows from [SV4, (7.14)]. The argument there does not obviously carry over to the twisted case, so we argue ab initio.

Proof. With $\hat{p}$ and $\hat{q}$ as in (10.9), $\hat{J}_{\alpha} \circ \hat{J}_{\alpha}=R \hat{q}_{!} \hat{p}^{*} R \hat{q}_{!} \hat{p}^{*}[2]$. Applying base change in the fiber square

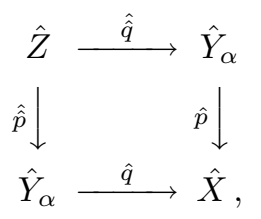


we can rewrite the previous identity as $\hat{J}_{\alpha} \circ \hat{J}_{\alpha}=R \hat{q}_{!} R \hat{\hat{q}}_{!} \hat{p}^{*} \hat{p}^{*}[2]$. Let $\hat{\pi}_{1}, \hat{\pi}_{2}$ denote the projections from $\hat{X} \times \hat{X}$ to the two factors, and $\pi: \hat{Z} \rightarrow \hat{X} \times \hat{X}$ given by $\hat{\pi}(\hat{z})=(\hat{p}(\hat{\hat{p}}(\hat{z})), \hat{q}(\hat{\hat{q}}(\hat{z})))$. Then

$$
\hat{J}_{\alpha} \circ \hat{J}_{\alpha}=R \hat{q}_{!} R \hat{\hat{q}}_{!} \hat{\hat{p}}^{*} \hat{p}^{*}[2]=R \hat{\pi}_{2 !} R \hat{\pi}_{!} \hat{\pi}^{*} \hat{\pi}_{1}^{*}[2] .
$$

The projection formula for $\pi: \hat{Z} \rightarrow \hat{X} \times \hat{X}$ asserts that $R \hat{\pi}_{!} \hat{\pi}^{*} \mathcal{S}=\left(R \hat{\pi}_{!} \mathbb{C}_{\hat{Z}}\right) \otimes \mathcal{S}$, for any sheaf $\mathcal{S}$ on $\hat{X} \times \hat{X}$. We use this formula to transform (10.41) into

$$
\left(\hat{J}_{\alpha} \circ \hat{J}_{\alpha}\right)(\mathcal{G})=R \hat{\pi}_{2 !}\left(\left(R \hat{\pi}_{!} \mathbb{C}_{\hat{Z}}\right) \otimes \hat{\pi}_{1}^{*}(\mathcal{G})\right)[2] .
$$

The geometry of the various fibrations gives

$$
\hat{\pi}^{-1}\left(\hat{x}_{1}, \hat{x}_{2}\right)= \begin{cases}\mathbb{C} & \text { if } \hat{x}_{1}=\hat{x}_{2}, \\ \mathbb{C}^{*} & \text { if } \hat{x}_{1} \text { and } \hat{x}_{2} \text { are in position } \alpha, \\ \emptyset & \text { otherwise }\end{cases}
$$

In the $K$-group, this gives the equality $R \hat{\pi}_{!} \mathbb{C}_{\hat{Z}}[2]=\mathbb{C}_{\Delta \hat{X}}$, and this equality persists even in the $K$-group of the $G_{\mathbb{R}}$-equivariant derived category. Thus $\left(\hat{J}_{\alpha} \circ \hat{J}_{\alpha}\right)(\mathcal{G})=$ $R \hat{\pi}_{2 !}\left(\left(R \hat{\pi}_{!} \mathbb{C}_{\hat{Z}}\right) \otimes \hat{\pi}_{1}^{*}(\mathcal{G})\right)[2]=R \hat{\pi}_{2 !}\left(\mathbb{C}_{\Delta \hat{X}} \otimes \hat{\pi}_{1}^{*}(\mathcal{G})\right)=\mathcal{G}$ in $K\left(D_{G_{\mathbb{R}}}(X)_{\lambda}\right)$.

Lemma 10.39 allows us to treat the points $x$ and $s_{\alpha} x$ in a symmetric fashion. Thus, without loss of generality, we assume

$$
\left|\alpha_{x}(t)\right| \leq 1 .
$$

Recall: the submanifolds $N^{\prime \prime}(t, x), N^{\prime \prime}\left(t, s_{\alpha} x\right)$ depended on the choice of $\Psi^{\prime \prime} \subset \Phi^{+}$. At $x$, we make the minimal choice

$$
\Psi^{\prime \prime}(x)=\left\{\beta \in \Phi^{+}|| \beta_{x}(t) \mid>1\right\},
$$

and at $s_{\alpha} x$, we also include $\alpha$ itself even if (10.44) is an equality, i.e.,

$$
\Psi^{\prime \prime}\left(s_{\alpha} x\right)=\Psi^{\prime \prime}(x) \cup\{\alpha\} .
$$

Because $\alpha$ is simple, and because of (10.44), this choice is consistent with (5.15b).

In defining the morphism (5.22), we think of $t \in \tilde{T}_{\mathbb{R}}$ as variable, and this is the reason for the appearance of the factor $\mathbb{C}_{\lambda}$. Now that we regard $t$ as fixed, we can evaluate sections of $\mathbb{C}_{\lambda}$ - which was constructed as a subsheaf of $\mathcal{O}_{\tilde{G}}$ - at $t$, giving us morphisms

$$
\phi\left(\mathcal{G}, s_{\alpha} x\right): t^{*} \mathcal{G}\left(s_{\alpha} x\right) \rightarrow \mathcal{G}\left(s_{\alpha} x\right), \quad \phi\left(\hat{J}_{\alpha} \mathcal{G}, x\right): t^{*}\left(\hat{J}_{\alpha} \mathcal{G}\right)(x) \rightarrow\left(\hat{J}_{\alpha} \mathcal{G}\right)(x) .
$$

The two sheaves are defined on different (open) Schubert cells, so we cannot relate the two morphisms directly. Our choices (10.45) imply that $N^{\prime \prime}\left(t, s_{\alpha} x\right)$ fibers over $N^{\prime \prime}(t, x)$ with fiber $\mathbb{C}$; for each $z \in N^{\prime \prime}(t, x)$, the inverse image in $N^{\prime \prime}\left(t, s_{\alpha} x\right)$ consists of all points in relative position $\alpha$ relative to $z$. Differently put, in the diagram (10.9), $p$ maps the inverse image $q^{-1}\left(N^{\prime \prime}(t, x)\right)$ isomorphically onto $N^{\prime \prime}\left(t, s_{\alpha} x\right)$. Thus, for economy of notation, we write the fibration as $q: N^{\prime \prime}\left(t, s_{\alpha} x\right) \rightarrow N^{\prime \prime}(t, x)$.

10.47 Lemma. The sheaves $\left.\left(\hat{J}_{\alpha} \mathcal{G}\right)(x)\right|_{N^{\prime \prime}(t, x)}$ and $R q_{!}\left(\left.\mathcal{G}\left(s_{\alpha} x\right)\right|_{N^{\prime \prime}\left(t, s_{\alpha} x\right)}\right)[1]$ are canonically isomorphic, so that

$$
\left.e^{\alpha_{x}}(t) \phi\left(\hat{J}_{\alpha} \mathcal{G}, x\right)\right|_{N^{\prime \prime}(t, x)}=R q_{!}\left(\left.\phi\left(\mathcal{G}, s_{\alpha} x\right)\right|_{N^{\prime \prime}\left(t, s_{\alpha} x\right)}\right)[1] .
$$


Proof. We consider the commutative diagram

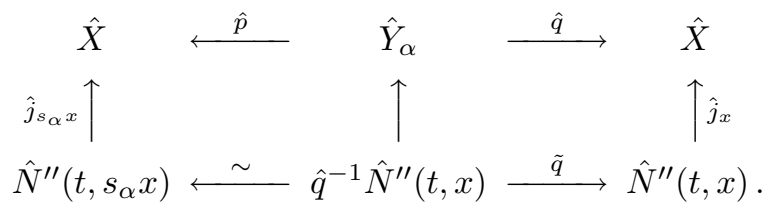

By base change in the right square,

$$
\hat{j}_{x}^{*} \hat{J}_{\alpha} \mathcal{G}=\hat{j}_{x}^{*} R \hat{q}_{!} \hat{p}^{*} \mathcal{G}[1] \simeq R \tilde{q}_{!} \hat{j}_{s_{\alpha} x}^{*} \mathcal{G}[1]
$$

in $\mathrm{D}_{\tilde{T}_{\mathbb{R}}}\left(N^{\prime \prime}(t, x)\right)_{s_{\alpha} \lambda}$. In $\S 5$, we constructed the morphisms $(10.46)$ from the $\tilde{T}_{\mathbb{R}^{-}}$ equivariant structure of the two sheaves on the two open Schubert cells. Restricting these morphisms to (the $\tilde{T}_{\mathbb{R}}$-invariant subsets) $N^{\prime \prime}\left(t, s_{\alpha} x\right)$ and $N^{\prime \prime}(t, x)$ is equivalent to applying the construction directly to the restricted sheaves $j_{s_{\alpha} x}^{*} \mathcal{G}$ and $j_{x}^{*} \hat{J}_{\alpha} \mathcal{G}$. Thus (10.49) relates the restricted morphisms. The construction of the first of the two morphisms (10.46) involves multiplication by $e^{\lambda_{s_{\alpha} x}-\rho_{s_{\alpha} x}}$, that of the second, multiplication by $e^{\left(s_{\alpha} \lambda\right)_{x}-\rho_{x}}$ - in both cases, these expressions are considered as functions on $\mathfrak{h} \cong \tilde{\mathfrak{t}}$; cf. (5.7)-(5.8). This accounts for the factor $e^{\alpha_{x}}(t)$ in the statement of the lemma: the value, at $t$, of the quotient of the two exponential expressions,

$$
e^{\left(s_{\alpha} \lambda\right)_{x}-\rho_{x}-\left(\lambda_{s_{\alpha} x}-\rho_{s_{\alpha} x}\right)}=e^{\alpha_{x}},
$$

which is well defined on all of $\tilde{T}_{\mathbb{R}}$.

Let us reformulate the remaining problem, stripping away the inessential aspects of our situation. We consider a complex vector space $V$ - in our case, $N^{\prime \prime}(t, x)$ identified with its Lie algebra via exp - and a linear transformation

$$
f: \mathbb{C} \times V \rightarrow \mathbb{C} \times V, \quad f(\mathbb{C}) \subset \mathbb{C}, \quad f(V) \subset V,
$$

such that

a) all eigenvalues $\zeta$ of $f$ on $V$ satisfy $|\zeta|>1$,

b) the eigenvalue of $f$ on $\mathbb{C}$ has absolute value $\geq 1$.

In the situation at hand, $\mathbb{C} \times V$ is $N^{\prime \prime}\left(t, s_{\alpha} x\right)$, again identified with its Lie algebra, and $f$ is the map induced by $t$; the hypotheses $(10.50 \mathrm{~b})$ are satisfied because of (10.45). Next, we consider a constructible sheaf $\mathcal{E} \in \mathrm{D}^{b}(\mathbb{C} \times V)$ together with the datum of a morphism

$$
\phi: f^{*} \mathcal{E} \rightarrow \mathcal{E},
$$

namely $\mathcal{E}=\left.\mathcal{G}\left(s_{\alpha} x\right)\right|_{N^{\prime \prime}\left(t, s_{\alpha} x\right)}$ and $\phi=\left.\phi\left(\mathcal{G}, s_{\alpha} x\right)\right|_{N^{\prime \prime}\left(t, s_{\alpha} x\right)}$. Let $q: \mathbb{C} \times V \rightarrow V$ be the projection. Then $R q_{!} \mathcal{E} \in \mathrm{D}^{b}(V)$ and $R q_{!} \phi: f^{*}\left(R q_{!} \mathcal{E}\right) \rightarrow R q ! \mathcal{E}$.

10.52 Lemma. Under the hypotheses just mentioned,

$\sum(-1)^{i} \operatorname{tr}\left(R q ! \phi: \mathrm{H}_{\{0\}}^{i}(R q ! \mathcal{E}) \rightarrow \mathrm{H}_{\{0\}}^{i}(R q ! \mathcal{E})\right)=\sum(-1)^{i} \operatorname{tr}\left(\phi: \mathrm{H}_{\{0\}}^{i}(\mathcal{E}) \rightarrow \mathrm{H}_{\{0\}}^{i}(\mathcal{E})\right)$.

We note that this completes the induction step for the fixed point theorems 5.12, 5.24, and 5.27: the present lemma, combined with Lemma 10.47 implies the identity (10.38). 
Proof of 10.52. We compactify $q$ to $\bar{q}: \mathbb{P}^{1} \times V \rightarrow V$ and identify $V \cong\{\infty\} \times V$. We write $j: \mathbb{C} \times V \hookrightarrow \mathbb{P}^{1} \times V$ for the open embedding, $\bar{k}: V \cong\{\infty\} \times V \hookrightarrow \mathbb{P}^{1} \times V$ for the closed embedding, $\bar{i}: \mathbb{P}^{1} \cong \bar{q}^{-1}(0) \hookrightarrow \mathbb{P}^{1} \times V$ for the natural inclusion, and $i$ for the restricted map $i: \mathbb{C} \hookrightarrow \mathbb{C} \times V$. Thus,

$$
R q ! \mathcal{E}=R \bar{q}_{!} j_{!} \mathcal{E}=R \bar{q}_{*} j_{!} \mathcal{E}
$$

and, by applying base change to the projection $\bar{q}$, we get

$$
\begin{aligned}
& \sum(-1)^{i} \operatorname{tr}\left(R q ! \phi: \mathrm{H}_{\{0\}}^{i}(R q ! \mathcal{E}) \rightarrow \mathrm{H}_{\{0\}}^{i}(R q ! \mathcal{E})\right) \\
& =\sum(-1)^{i} \operatorname{tr}\left(\phi: \mathrm{H}^{i}\left(\mathbb{P}^{1}, \bar{i}^{!} j_{!} \mathcal{E}\right) \rightarrow \mathrm{H}^{i}\left(\mathbb{P}^{1}, \bar{i}^{!} j_{\mathfrak{L}} \mathcal{E}\right)\right) .
\end{aligned}
$$

The Grothendieck-Lefschetz fixed point formula expresses the right hand side of (10.54) as a sum of two terms associated to the fixed points 0 and $\infty$ of $f$ on $\mathbb{P}^{1} \cong \bar{q}^{-1}(0)$; see $[\mathrm{KSa}, \S 9.6]$ for an exposition of this formalism. We use the fixed point formula of $[\mathrm{GM}]$ to compute these contributions. We will deal with the point 0 first. According to (10.50b) we can view the map $f$ (restricted to $\mathbb{P}^{1}$ ) as expanding around 0 . Theorem 4.7 in $[\mathrm{GM}]$ gives the fixed point contribution from 0 in five different ways. One of these, involving the complex which Goresky-MacPherson call $\mathbf{A}_{4}$, "shrieks" the sheaf $i^{!} j_{!} \mathcal{E}$ to the contacting directions - in our case, this means taking local cohomology at $\{0\}$ - then restricts the resulting sheaf to the fixed point - which, in our case, is a vacuous operation. Hence

$$
\begin{gathered}
\sum(-1)^{i} \operatorname{tr}\left(\phi: \mathrm{H}_{\{0\}}^{i}\left(\mathbb{P}^{1}, \bar{i}^{!} j ! \mathcal{E}\right) \rightarrow \mathrm{H}_{\{0\}}^{i}\left(\mathbb{P}^{1}, \bar{i}^{!} j ! \mathcal{E}\right)\right) \\
=\sum(-1)^{i} \operatorname{tr}\left(\phi: \mathrm{H}_{\{0\}}^{i}\left(\mathbb{C}, i^{!} \mathcal{E}\right) \rightarrow \mathrm{H}_{\{0\}}^{i}\left(\mathbb{C}, i^{\prime} \mathcal{E}\right)\right) \\
=\sum(-1)^{i} \operatorname{tr}\left(\phi: \mathrm{H}_{\{0\}}^{i}(\mathcal{E}) \rightarrow \mathrm{H}_{\{0\}}^{i}(\mathcal{E})\right)
\end{gathered}
$$

i.e., the right hand side of the identity (10.52), is the contribution of the fixed point 0 to the global trace (10.54). We had argued initially that this global trace coincides with the left hand side in 10.52. To finish the proof of the proposition, we need to show that the fixed point contribution at $\infty$ in (10.54) is zero. The map $f: \mathbb{P}^{1} \rightarrow \mathbb{P}^{1}$ contracts around infinity, so "shrieking" to the contracting directions becomes vacuous, and restricting to the fixed point means taking the stalk. Thus

$$
\sum(-1)^{i} \operatorname{tr}\left(\phi: \mathrm{H}^{i}\left((\bar{i} ! j ! \mathcal{E})_{\{\infty\}}\right) \rightarrow \mathrm{H}^{i}\left((\bar{i} ! j ! \mathcal{E})_{\{\infty\}}\right)\right)
$$

is the fixed point contribution at infinity. This is also the fixed point contribution at $(\infty, 0)$ for the pair $\left(j ! \mathcal{E}, j_{!} \phi\right)$ and the map $\bar{f}: \mathbb{P}^{1} \times V \rightarrow \mathbb{P}^{1} \times V$ induced by $f$; to see this, note that around $(\infty, 0), \bar{f}$ is contracting only in the direction of $\mathbb{P}^{1}$. We now use one of the other formulas in $[\mathrm{GM}]$ to get an alternate expression for the fixed point contribution at $(\infty, 0)$. The complex $\mathbf{A}_{5}$ in $[\mathrm{GM}]$ is obtained by restricting the sheaf $j ! \mathcal{E}$ to the expanding directions - in our case $\{\infty\} \times V$ - then "shrieking" to the fixed point. But $j_{!} \mathcal{E}$ is the sheaf $\mathcal{E}$ extended by 0 along $\{\infty\} \times V$. In the present setting, then, the entire complex $\mathbf{A}_{5}$ reduces to 0, so (10.56) vanishes.

\section{REFERENCES}

[A] M.Atiyah, Characters of semisimple Lie groups, lecture notes (1976), reprinted in Collected works of Michael Atiyah, vol 4, pp 491-557, Springer 1988. MR 91h:01094d

[B] A.Beilinson, Localization of representations of reductive Lie algebras, Proceedings of the International Congress of Mathematicians, Vol. 1, 2 (Warsaw, 1983), PWN, Warsaw, 1984, pp. 699-710. MR 86m:17007

[BB1] A.Beilinson and J.Bernstein, Localisations de $\mathfrak{g}-$ modules, C. R. Acad. Sci. Paris 292 (1981), 15-18. MR 82k:14015 
[BB2] A.Beilinson and J.Bernstein, A generalization of Casselman's submodule theorem, in: Representation Theory of Reductive Groups, Progress in Mathematics, Vol 40, Birkhäuser, Boston, 1983, pp. 35-52. MR 85e:22024

[BB3] A.Beilinson and J.Bernstein, Proof of Jantzen's conjecture, Advances in Soviet Mathematics 16 (1993), 1-50. MR 95a:2202

[BL] J.Bernstein and V.Lunts, Equivariant sheaves and functors, Lecture Notes in Mathematics 1578, Springer, 1994. MR 95k:55012

[BM] E.Bierstone and P.D.Milman, Semi-analytic and subanalytic sets, Publications Math. IHES 67 (1988), 5-42. MR 89k:32011

[BV] N.Berline and M.Vergne, Classes caracteristiques equivariantes. Formule de localisation en cohomologie equivariante, C. R. Acad. Sci. Paris Sér. I Math. 295 (1982), 539-541. MR 83m:58002

[C] J-Y.Charbonnel, Sur les semi-caracteres des groupes de Lie resolubles connexes, J. Funct. Anal. 41 (1981), 175-203. MR 83c:22011

[D1] M.Duflo, Representations of the fundamental series of a semisimple Lie group, Funkcional. Anal. i Priložen 4 (1970), 38-42 (Russian). MR 45:6975

[D2] M.Duflo, Théorie de Mackey pour les groupes de Lie algèbriques, Acta Math. 149 (1982), 153-213. MR 85h:22022

[FS] A.I.Fomin and N.N.Šhapovalov, A certain property of the characters of irreducible representations of real semisimple Lie groups, Funkcional. Anal. i Priložen 8 (1974), 87-88 (Russian). MR 51:3357

[GM] M. Goresky and R. MacPherson, Local contribution to the Lefschetz fixed point formula, Inventiones Math. 111 (1993), 1-33. MR 94b:55009

[GS] P. Griffiths and W. Schmid, Locally homogeneous complex manifolds, Acta Math. 123 (1969), 253-302. MR 41:4587

[HC1] Harish-Chandra, On some applications of the universal enveloping algebra of a semisimple Lie algebra., Trans. AMS 70 (1951), 28-96. MR 13:428c

[HC2] Harish-Chandra, Representations of a semisimple Lie group on a Banach space I, Trans. AMS 75 (1953), 185-243. MR 15:100f

[HC3] Harish-Chandra, Representations of a semisimple Lie group III, Trans. AMS 76 (1954), 234-253. MR 16:11e

[HC4] Harish-Chandra, Invariant Eigendistributions on a Semisimple Lie Group, Trans. AMS 119 (1965), 457-508. MR 31:4862d

[HC5] Harish-Chandra, Discrete series for semisimple Lie groups I, Acta Math. 113 (1965), 241-318. MR 36:2744

[HC6] Harish-Chandra, Discrete series for semisimple Lie groups II, Acta Math. 116 (1966), 1-111. MR 36:2745

[HC7] Harish-Chandra, Harmonic analysis on real reductive groups $I$, the theory of the constant term, Jour. Func. Analysis 19 (1975), 104-204. MR 53:3201

[Hec] H.Hecht, Characters of some representations of Harish-Chandra, Math. Annalen 219 (1976), 213-226. MR 55:573

[HMSW1] H.Hecht, D.Miličić, W.Schmid and J.Wolf, Localization and standard modules for real semisimple Lie groups I: The duality theorem, Invent. Math. 90 (1987), 297-332. MR 89e: 22025

[HMSW2] H.Hecht, D.Miličić, W.Schmid and J.Wolf, Localisation and standard modules for real semisimple Lie groups II: Irreducibility, vanishing theorems and classification, preprint.

[HS] H.Hecht and W.Schmid, Characters, asymptotics, and $\mathfrak{n}$-homology of Harish-Chandra modules, Acta Math. 151 (1983), 49-151. MR 84k:22026

[Hel] S.Helgason, Differential Geometry, Lie Groups, and Symmetric Spaces, Academic Press, 1978. MR 80k:53081

[Hi] T.Hirai, The characters of some induced representations of Lie groups, J. Math. Kyoto Univ. 8 (1968), 313-363. MR 39:354

[Hn1] H.Hironaka, Resolution of singularities of an algebraic variety over a field of characteristic zero: I, II, Ann. of Math. 79 (1964), 109-326. MR 33:7333

[Hn2] H.Hironaka, Subanalytic sets, Number Theory, Algebraic Geometry, and Commutative Algebra, Kinokuniya, Tokyo, 1973, pp. 453-493. MR 51:13275 
[HK] R.Hotta and M.Kashiwara, The invariant holonomic system on a semisimple Lie algebra, Inventiones Math. 75 (1984), 327-358. MR 87i:22041

[Ka1] M.Kashiwara, The Riemann-Hilbert problem for holonomic systems, Publ. RIMS, Kyoto University 20 (1984), 319 - 365. MR 86j:58142

[Ka2] M.Kashiwara, Index theorem for constructible sheaves, Astérisque 130 (1985), 193 209. MR 87f:58160

[Ka3] M.Kashiwara, Open problems in group representation theory, Proceedings of Taniguchi Symposium held in 1986, RIMS preprint 569, Kyoto University, 1987.

[Ka4] M.Kashiwara, Character, character cycle, fixed point theorem, and group representations, Advanced Studies in Pure Mathematics, vol. 14, Kinokuniya, Tokyo, 1988, pp. 369-378. MR 91h:22029

[Ka5] M.Kashiwara, The invariant holonomic system on a semisimple Lie group, Algebraic analysis, Vol. I, Academic Press, Boston, 1988, pp. 277-286. MR 90k:22021

[Ka6] M.Kashiwara, D-modules and Representation Theory of Lie Groups, RIMS preprint 940, Kyoto University, 1993.

[KSa] M.Kashiwara and P.Schapira, Sheaves on manifolds, Springer, 1990.

[KSd] M.Kashiwara and W.Schmid, Quasi-equivariant D-modules, equivariant derived category, and representations of reductive Lie groups, Lie Theory and Geometry, in Honor of Bertram Kostant, Progress in Mathematics, Birkhäuser, 1994, pp. 457-488.

[Ki1] A.A.Kirillov, Characters of unitary representations of Lie groups, Funkcional. Anal. i Priložen 2 (1968), 40-55 (Russian). MR 38:4615

[Ki2] A.A.Kirillov, Characters of unitary representations of Lie groups. Reduction theorems, Funkcional. Anal. i Priložen 3 (1969), 36-47 (Russian). MR 40:1538

[Ki3] A.A.Kirillov, Elements of the Theory of Representations, Springer, 1976. MR 54:447

[Ma] T.Matsuki, Orbits on affine symmetric spaces under the action of parabolic subgroups, Hiroshima Math. Jour. 12 (1982), 307-320. MR 83k:53072

[Me] Z.Mebkhout, Une équivalence de catégories - Une autre équivalence de catégories, Comp. Math. 51 (1984), 55 - 64. MR 85k:58072; MR 85k:58073

[Mi] D.Miličić, Localization and Representation Theory of Reductive Lie Groups, preliminary manuscript (available at www.math.utah.edu/ ${ }^{\sim}$ milicic/), 1993.

[MUV] I.Mirković, T.Uzawa, and K.Vilonen, Matsuki Correspondence for Sheaves, Inventiones Math. 109 (1992), 231-245. MR 93k:22011

[MV] I.Mirković and K.Vilonen, Characteristic varieties of character sheaves, Inventiones Math. 93 (1988), 405-418. MR 89i:20066

[Os] M.S.Osborne, Lefschetz formulas on nonelliptic complexes, Thesis, Yale University 1972. Reprinted in: Representation Theory and Harmonic Analysis on Semisimple Lie Groups, Mathematical Surveys and Monographs 31, AMS, Providence 1989, pp 171222. MR 90k:22022

[OM] T.Oshima and T.Matsuki, A description of discrete series for semisimple symmetric spaces, Advanced Studies in Pure Math. 4 (1984), 331-390. MR 87m:22042

[R1] W. Rossmann, Kirillov's Character Formula for Reductive Lie Groups, Inventiones Math. 48 (1978), 207-220. MR 81g:22012

[R2] W.Rossmann, Characters as contour integrals, Lecture Notes in Mathematics 1077, Springer, 1984, pp. 375-388. MR 86e:22024

[R3] W.Rossmann, Invariant Eigendistributions on a Semisimple Lie Algebra and Homology Classes on the Conormal Variety I, II, Jour. Func. Anal. 96 (1991), 130-193. MR 92g:22033; MR 92g:22034

[S1] W.Schmid, Homogenous complex manifolds and representations of semisimple Lie groups, thesis, Berkeley 1967. Reprinted in "Representation theory and harmonic analysis on semisimple Lie groups", Mathematical Surveys and Monographs 31, AMS, Providence, RI, 1989, 223-286. MR 90i:22025

[S2] W.Schmid, Some properties of square-integrable representations of semisimple Lie groups, Ann. of Math. 102 (1975), 535-564. MR 58:28303

[S3] W.Schmid, Two character identities for semisimple Lie groups, Non-Commutative Harmonic Analysis, Marseille-Luminy 1976, Lecture Notes in Mathematics 1578, Springer, 1977, pp. 196-225. MR 58:22405 
[S4] W.Schmid, Construction and classification of irreducible Harish-Chandra modules, Harmonic Analysis on Reductive Groups, Progress in Mathematics 101, Birkhäuser, pp. 235-275. MR 93j:22028

[S5] W.Schmid, Character formulas and localization of integrals, Proceedings of the conference on symplectic geometry and mathematical physics, Ascona 1996, to appear.

[SV1] W.Schmid and K.Vilonen, Characters, Fixed Points and Osborne's Conjecture, Representation Theory of Groups and Algebras, Contemp. Math. 145, 1993, pp. 287-303. MR 95a:22020

[SV2] W.Schmid and K.Vilonen, Weyl group actions on Lagrangian cycles and Rossmann's formula, Proceedings of NATO Advanced Research Conference on Mathematical Physics and Group Theory, Kluwer Academic Press, 1994, pp. 242-250. CMP 95:05

[SV3] W.Schmid and K.Vilonen, Characters, characteristic cycles and nilpotent orbits, Geometry, Topology, and Physics for Raoul Bott, International Press, 1995, pp. 329-340. MR 96g:22021

[SV4] W.Schmid and K.Vilonen, Characteristic cycles of constructible sheaves, Inventiones Math. 124 (1996), 451-502. MR 96k:32016

[SW] W.Schmid and J.Wolf, Geometric quantization and derived functor modules for semisimple Lie groups, Jour. Funct. Anal. 90 (1990), 48-112. MR 91j:22012

[V] D.Vogan, The algebraic structure of the representations of semisimple Lie groups I, Ann. of Math. 109 (1979), 1-60. MR 81j:22020

[W] J.Wolf, The action of a real semisimple group on a complex flag manifold. I. Orbit structure and holomorphic arc components, Bull. Amer. Math. Soc. 75 (1969), 11211237. MR 40:4477

Department of Mathematics, Harvard University, Cambridge, Massachusetts 02138

E-mail address: schmid@math.harvard.edu

Department of Mathematics, Brandeis University, Waltham, Massachusetts 02254

E-mail address: vilonen@math.brandeis.edu 